\title{
Combination of $\mathrm{CN}(1-0), \mathrm{HCN}(1-0)$, and $\mathrm{HNC}(1-0)$ : A possible indicator for a high-mass star formation sequence in the Milky Way ${ }^{\star}$
}

\author{
X. H. Han ${ }^{1,2}$, J. J. Zhou ${ }^{1,3}$, J. Z. Wang ${ }^{4}$, J. Esimbek ${ }^{1,3}$, J. S. Zhang ${ }^{5}$, and N. Wang ${ }^{1,3}$ \\ ${ }^{1}$ Xinjiang Astronomical Observatory, CAS, 150, Science 1-street, Urumqi, 830011 Xinjiang, PR China \\ e-mail: hanxh@xao.ac.cn \\ 2 Graduate University of Chinese Academy of Sciences, 19A Yuquan Road, 100049 Beijing, PR China \\ 3 Key Laboratory of Radio Astronomy, Chinese Academy of Sciences, 830011 Nanjing, 210008 Jiangsu, PR China \\ 4 Shanghai Astronomical Observatory, Chinese Academy of Sciences, 80 Nandan Road, 200030 Shanghai, PR China \\ 5 Center for astrophysics, Guangzhou University, 510006 Guangzhou, PR China
}

Received 11 March 2014 / Accepted 26 January 2015

\begin{abstract}
Context. $\mathrm{CN}, \mathrm{HCN}$, and $\mathrm{HNC}$ have been used to discuss the star formation sequence in galaxies, but recent studies of large samples involving these lines did not yet provide convincing results.

Aims. We intend to determine whether the global line ratios $\mathrm{CN} / \mathrm{HCN}, \mathrm{CN} / \mathrm{HNC}$, and $\mathrm{HCN} / \mathrm{HNC}$ can be used to trace the high-mass star formation sequence in the Milky Way.

Methods. We performed map observations of $\mathrm{CN}(1-0), \mathrm{HCN}(1-0)$, and $\mathrm{HNC}(1-0)$ toward 38 high-mass star-forming regions, which includes high-mass starless cores (HMSC), high-mass protostars (HMPO), UCHII (ultra-compact HII) and normal HII regions. We identified the molecular clumps associated with them, and removed the clumps that were affected by environment. We averaged all the detected emission from each clump to obtain global line ratios and investigated their variations with the evolutionary stages of high-mass star-forming clumps.

Results. The global line ratios of $I_{\mathrm{CN}} / I_{\mathrm{HNC}}$ and $I_{\mathrm{HCN}} / I_{\mathrm{HNC}}$ for HMSC clumps (HMSCCs), HMPO clumps (HMPOCs), UCHII region clumps (UCHIICs), and HII region clumps display an increasing trend. We tentatively divide the star-forming regions into two types. Type 1 sources include HMSCCs and HMPOCs that are not associated with external $20 \mathrm{~cm}$ continuum emission. Type 2 sources include all UCHIICs and HIICs. Our analysis shows that the $I_{\mathrm{CN}} / I_{\mathrm{HNC}}$ and $I_{\mathrm{HCN}} / I_{\mathrm{HNC}}$ line ratios can trace the evolution from type 1 to type 2 well. The same method may be used to study the evolution of external galaxies.

Conclusions. $I_{\mathrm{CN}} / I_{\mathrm{HNC}}$ and $I_{\mathrm{HCN}} / I_{\mathrm{HNC}}$ appear to be good tracers for the evolution of high-mass star-forming regions in the Milky Way.
\end{abstract}

Key words. ISM: clouds - ISM: molecules

\section{Introduction}

The formation and the early evolution of high-mass stars are still only poorly understood (Zinnecker \& Yorke 2007; Tan et al. 2014), while chemical studies on the different evolutionary stages of high-mass star formation provide an important tool for investigating its underlying physical processes (Gerner et al. 2014). HCN is a widely used dense gas tracer, $\mathrm{CN}$ is a good tracer of photodissociation regions (PDRs) near massive young stars (Rodriguez et al. 1998), and HNC is a reliable tracer of cold gas (Schilke et al. 1992). The abundances of CN, HCN, and HNC are sensitive to temperature, optical depth, and UV radiation (Schilke et al. 1992; Fuente et al. 1993; Boger \& Sternberg 2005). To a significant extent, $\mathrm{CN}$ is a photodissociation product of $\mathrm{HCN}$ and $\mathrm{HNC}$, and the abundance ratio of $\mathrm{CN} / \mathrm{HCN}$ increases in the outer regions of molecular clouds that are exposed to UV radiation (Baan et al. 2008 and references therein). Mapping observation studies of the Orion Bar and the reflection nebulae NGC 2023 and NGC 7023 suggest that the CN/HCN intensity ratios are highest near the stellar sources that illuminate far-ultraviolet (FUV) radiation fields, while the ratios decrease

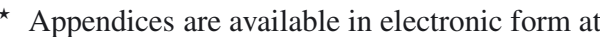
http: //www . aanda . org
}

with increasing optical depth and distance from the stars (Boger \& Sternberg 2005). This behavior is broadly consistent with theoretical expectations (Sternberg \& Dalgarno 1995; Jansen et al. 1995) and is evidence for selective photodissociation of HCN versus CN (van Zadelhoff et al. 2003; Thi et al. 2004). On the other hand, HNC transfers to $\mathrm{HCN}$ at high temperature, and so the abundance ratio $\mathrm{HCN} / \mathrm{HNC}$ is strongly dependent on the temperature (Vasyunina et al. 2011; Ungerechts et al. 1997). The abundance ratio $\mathrm{HCN} / \mathrm{HNC}$ is $\sim 1$ for infrared dark clouds (IRDCs; Vasyunina et al. 2011), and is higher and reaches up to 13 for high-mass protostars (HMPOs; Helmich \& van Dishoeck 1997 ), and the ratio is $\sim 80$ for very warm regions like Orion (Schilke et al. 1992; Goldsmith et al. 1986). All these suggest that the combination of $\mathrm{CN}, \mathrm{HCN}$, and $\mathrm{HNC}$ is a promising indicator of the star formation sequence in galaxies.

$\mathrm{CN}, \mathrm{HCN}$, and HNC have been used to discuss the star formation sequence in samples of external galaxies (Aalto et al. 2002; Baan et al. 2008, 2010; Costagliola et al. 2011; Aalto 2013). Aalto et al. (2002) found abundance ratios of $\mathrm{CN} / \mathrm{HCN}>1$ for three starburst galaxies, indicating that a significant part of the interstellar medium (ISM) in these galaxies resides in PDRs, and $\mathrm{CN} / \mathrm{HCN}<1$ in four active galactic nuclei (AGNs). This suggests that the ISM of these galaxies 
contains a large amount of warm dense gas, which implies that the galaxies may be in an early stage of their starburst development. Costagliola et al. (2011) also found that luminous infrared galaxies (LIRGs) with $\mathrm{HC}_{3} \mathrm{~N}(10-9)$ detected have intensity ratios of $\mathrm{HCO}^{+} / \mathrm{HCN}<1$ and $\mathrm{HNC} / \mathrm{HCN}>0.4$. Starburst galaxies tend to have higher $\mathrm{HCO}^{+} / \mathrm{HCN}$ and lower $\mathrm{HNC} / \mathrm{HCN}$ intensity ratios.

Recently, many authors have focused on the chemical evolution of high-mass star-forming regions in the Milky Way (Vasyunina et al. 2011; Sanhueza et al. 2012; Hoq et al. 2013; Gerner et al. 2014). CN, HCN, and HNC are usually included in these studies. However, Vasyunina et al. (2011) did not detect any significant differences in the $\mathrm{HCN} / \mathrm{HNC}$ abundance ratios between "quiescent", "middle", and "active" regions within their IRDC (infrared dark cloud) sample, whereas Gerner et al. (2014) obtained differences between IRDC, HMPO, HMC (hot molecular core), and UCHII regions with an $\mathrm{HCN} / \mathrm{HNC}$ abundance ratio between 0.3-0.6. Hoq et al. (2013) also found that integrated intensity ratios of $\mathrm{HCN}(1-0) / \mathrm{HNC}(1-0)$ show marginal evidence of an increase as the clumps evolve, with median values of 1.07, 1.19, and 1.64 for quiescent, protostellar, and HII/PDR sources, respectively. It is evident that these results are not conclusive as to whether the combination of $\mathrm{CN}(1-0), \mathrm{HCN}(1-0)$, and $\mathrm{HNC}(1-0)$ can be used to trace the evolution of high-mass star formation. One possible reason is that these three lines are affected by the environment because they are sensitive to optical depth, UV radiation, and shocks. On the other hand, these studies were based on single-point line data, which is not suitable for tracing chemical evolution of the whole high-mass star-forming regions. Since star formation takes place in dense molecular clumps, the global physical and chemical conditions of the clump will change as it evolves. Mapping observations of $\mathrm{CN}, \mathrm{HCN}$, and HNC for a large sample of high-mass starforming regions at different stages are needed to obtain their global physical and chemical properties and to help in determining whether the combinations of $\mathrm{CN}, \mathrm{HCN}$, and $\mathrm{HNC}$ can be used as indicators for the star formation sequence in the Milky Way, as well as to understand the star formation sequence in external galaxies.

Our objective in this paper is to determine whether the global integrated intensity ratios $\mathrm{CN} / \mathrm{HCN}, \mathrm{CN} / \mathrm{HNC}$, and $\mathrm{HCN} / \mathrm{HNC}$ increase with the age of embedded stars. We perform $\mathrm{CN}(1-$ $0), \mathrm{HCN}(1-0)$, and $\mathrm{HNC}(1-0)$ mapping observations for a large sample of high-mass star-forming regions at different evolutionary stages using the $13.7 \mathrm{~m}$ telescope of the Purple Mountain Observatory (PMO). In Sect. 2, we describe the sample we used, observation, archival data and data reduction, and the results are given in Sect. 3. We discuss the global line ratios of high-mass star-forming regions at different evolutionary stages and their variations in Sect. 4. Our conclusions are summarized in Sect. 5.

\section{Observation and data}

\subsection{PMO 13.7 m observation}

Table 1 lists all sources used in this paper. Nine high-mass starless cores (HMSCs) were selected from the lists of Sridharan et al. (2005) and Sakai et al. (2008). The sources were detected at $1.2 \mathrm{~mm}$, but without any infrared radiation at $8 \mu \mathrm{m}$. Eleven high-mass protostars (HMPOs) were selected from the list of Sridharan et al. (2002). These sources have no or very weak radio continuum emission. In addition, eleven UCHII (ultracompact HII) regions were chosen from the list of Bronfman et al. (1996). They show strong CS(2-1) emission, indicating that these sources are still embedded in dense gas envelopes.
Table 1. List of sources.

\begin{tabular}{|c|c|c|c|c|c|c|c|c|}
\hline \multirow{2}{*}{$\begin{array}{l}\text { Source } \\
\text { G22.35+0.41-1 }\end{array}$} & \multicolumn{3}{|c|}{$\mathrm{RA}(\mathrm{J} 2000)$} & \multicolumn{3}{|c|}{$\operatorname{Dec}(\mathrm{J} 2000)$} & \multirow{2}{*}{$\frac{\text { Evolution stage }}{\text { HMSC }}$} & \multirow{2}{*}{$\frac{\text { Ref }}{1}$} \\
\hline & 18 & 30 & 24.4 & -09 & 10 & 34 & & \\
\hline G23.60+0.00-2 & 18 & 34 & 21.1 & -08 & 18 & 07 & HMSC & 1 \\
\hline $\mathrm{G} 34.43+0.24-3$ & 18 & 53 & 20.4 & 01 & 28 & 23 & HMSC & 1 \\
\hline I18151-1208-2 & 18 & 17 & 50.3 & -12 & 07 & 54 & HMSC & 2 \\
\hline I18223-1243-3 & 18 & 25 & 08.3 & -12 & 45 & 28 & HMSC & 2 \\
\hline I18306-0835-3 & 18 & 33 & 32.1 & -08 & 32 & 29 & HMSC & 2 \\
\hline I18310-0825-4 & 18 & 33 & 39.5 & -08 & 21 & 10 & HMSC & 2 \\
\hline I18337-0743-3 & 18 & 36 & 18.2 & -07 & 41 & 01 & HMSC & 2 \\
\hline I18530+0215-2 & 18 & 55 & 29.0 & 02 & 17 & 43 & HMSC & 2 \\
\hline $\mathrm{I} 05358+3543$ & 05 & 39 & 10.4 & 35 & 45 & 19 & HMPO & 3 \\
\hline I18445-0222 & 18 & 47 & 10.8 & -02 & 19 & 06 & HMPO & 3 \\
\hline I1 $8517+0437$ & 18 & 54 & 13.8 & 04 & 41 & 32 & HMPO & 3 \\
\hline I20126+4104 & 20 & 14 & 26.0 & 41 & 13 & 32 & HMPO & 3 \\
\hline I20293+3952 & 20 & 31 & 10.7 & 40 & 03 & 10 & HMPO & 3 \\
\hline $\mathrm{I} 20343+4129$ & 20 & 36 & 07.1 & 41 & 40 & 01 & HMPO & 3 \\
\hline I $22134+5834$ & 22 & 15 & 09.1 & 58 & 49 & 09 & HMPO & 3 \\
\hline I22551+6221 & 22 & 57 & 05.2 & 62 & 37 & 44 & HMPO & 3 \\
\hline I $22570+5912$ & 22 & 59 & 06.5 & 59 & 28 & 28 & HMPO & 3 \\
\hline $\mathrm{I} 23033+5951$ & 23 & 05 & 25.7 & 60 & 08 & 08 & HMPO & 3 \\
\hline I23139+5939 & 23 & 16 & 09.3 & 59 & 55 & 23 & HMPO & 3 \\
\hline $\mathrm{I} 01202+6133$ & 01 & 23 & 32.1 & 61 & 48 & 49 & UCHII & 4 \\
\hline I03035+5819 & 03 & 07 & 25.7 & 58 & 30 & 53 & UCHII & 4 \\
\hline I19078+0901 & 19 & 10 & 15.2 & 09 & 06 & 22 & UCHII & 4 \\
\hline I19097+0847 & 19 & 12 & 11.3 & 08 & 52 & 21 & UCHII & 4 \\
\hline $\mathrm{I} 19111+1048$ & 19 & 13 & 27.9 & 10 & 53 & 34 & UCHII & 4 \\
\hline I19442+2427 & 19 & 46 & 19.9 & 24 & 35 & 24 & UCHII & 4 \\
\hline $\mathrm{I} 20081+3122$ & 20 & 10 & 09.1 & 31 & 31 & 34 & UCHII & 4 \\
\hline I23116+6111 & 23 & 13 & 45.4 & 61 & 28 & 18 & UCHII & 4 \\
\hline $\mathrm{I} 23133+6050$ & 23 & 15 & 31.5 & 61 & 07 & 07 & UCHII & 4 \\
\hline I23138+5945 & 23 & 16 & 04.8 & 60 & 01 & 59 & UCHII & 4 \\
\hline U49.49-0.37 & 19 & 23 & 40.1 & 14 & 31 & 05 & UCHII & 5 \\
\hline C41.1-0.21 & 19 & 07 & 10.22 & 07 & 09 & 46.5 & HII & 5 \\
\hline C49.00-0.30 & 19 & 22 & 26.02 & 14 & 07 & 18.1 & HII & 5 \\
\hline C48.93-0.29 & 19 & 22 & 16.28 & 14 & 03 & 50.4 & HII & 5 \\
\hline C49.38-0.30 & 19 & 23 & 11.95 & 14 & 27 & 31.2 & HII & 5 \\
\hline C51.36-0.00 & 19 & 26 & 00.12 & 16 & 20 & 28.7 & HII & 5 \\
\hline C52.94-0.59 & 19 & 31 & 18.69 & 17 & 26 & 47.3 & HII & 5 \\
\hline D53.64+0.24 & 19 & 29 & 41.23 & 18 & 27 & 17.8 & HII & 5 \\
\hline
\end{tabular}

Notes. From left to right, the columns are source name, right ascension, declination, the evolutionary stage of the source, and references, where the numbering in the references denotes (1) Sakai et al. (2008); (2) Sridharan et al. (2005); (3) Sridharan et al. (2002); (4) Bronfman et al. (1996); and (5) Anderson \& Bania (2009).

Finally, seven HII regions were selected from the list of Anderson \& Bania (2009).

We performed map observations of $\mathrm{CN}(1-0), \mathrm{HCN}(1-0)$, and HNC(1-0) toward all sources using the PMO $13.7 \mathrm{~m}$ telescope in March 2011, the corresponding rest frequencies are 113.4909 GHz, 88.63184 GHz, and $90.6635 \mathrm{GHz}$, respectively. The main beam size of the telescope is about 55". A cryogenically cooled nine-beam SIS receiver working in the $80-115 \mathrm{GHz}$ band in single-sideband (SSB) mode was employed for the observations as front end. The properties of the SIS mixers are introduced in Shan et al. (2010). FFTS spectrometers were used as back ends, which have a total bandwidth of $1 \mathrm{GHz}$ and 16384 channels, corresponding to a velocity resolution of $0.16,0.21$, and $0.20 \mathrm{~km} \mathrm{~s}^{-1}$ for CN(1-0), HCN (1-0), and HNC (1-0), respectively. The main beam efficiency is about 0.48 . The pointing accuracy of the telescope is better than $4^{\prime \prime}$. The typical system temperature in SSB mode is around $110 \mathrm{~K}$ and varies by about $10 \%$ for each beam. The on-the-fly (OTF) observing mode was applied to our observation, the map size is $10^{\prime} \times 10^{\prime}$, and the typical $\mathrm{rms}$ is about $0.1 \mathrm{~K}$ in $T_{A}^{*}$. The data processing was conducted using the Gildas package ${ }^{1}$.

All mapping regions of HMSCs and HMPOs have been studied in detail at different wavelengths (Beuther et al. 2002; Sakai et al. 2008; Sridharan et al. 2002, 2005), which makes

1 http://www.iram.fr/IRAMFR/GILDAS 
it straightforward to find star-forming objects in these regions and identify their evolutionary stages by referring to the relevant literature. We determined the unknown evolutionary stages for a few star-forming objects by checking the infrared and radio continuum emission at $\mathrm{cm}$ wavelengths using the methods proposed by Sakai et al. (2008), Sridharan et al. (2002, 2005). Detailed information about continuum emission data is given in Sect. 2.2.

\subsection{Archive data of $6(3.6) \mathrm{cm}$ and $20 \mathrm{~cm}$ continuum emission}

To assist in determining the evolutionary stages of HMSCs, HMPOs, and UCHII regions in the clumps, we obtained Midcourse Space Experiment (MSX) data at $8 \mu \mathrm{m}$ from the NASA/IPAC infrared science archive and radio continuum emission data at $6(3.6) \mathrm{cm}$ from the VLA. We obtained the $6 \mathrm{~cm}$ continuum emission map for sources without VLA data from the Multi-Array Galactic Plane Survey (MAGPIS) ${ }^{2} .20 \mathrm{~cm}$ continuum data from the NRAO VLA Sky Survey (NVSS) ${ }^{3}$ were also used to identify any HII regions close to the molecular clumps.

The VLA data were obtained from the NRAO DATA Archive System $^{4}$. The data processing was conducted using AIPS. Depending on the intensity of the sources, the integration time for the observations ranges from several minutes to several hours, and the resulting rms noise level ranges from 0.01 to 26 mJy beam $^{-1}$. Table A.2 summarizes the detailed information of the VLA archival data. The linear size of the continuum source was derived from its major axis. The coordinates of the continuum sources are given in Table A.1.

MAGPIS collects bits and pieces of the Galactic survey that have been imaged at high resolution. It includes the $6 \mathrm{~cm}$ and $20 \mathrm{~cm}$ VLA radio images and catalogs (White et al. 2005), as well as the main MAGPIS database of high-dynamic range, high-sensitivity VLA images for the region $5^{\circ}<l<48.5^{\circ}$, $|b|<0.8^{\circ}$ (Helfand et al. 2006). It has a synthesized beam of $\sim 6.2^{\prime \prime} \times 5.4^{\prime \prime}$ and a typical $\mathrm{rms}$ of $\sim 0.3 \mathrm{mJy} /$ beam (Helfand et al. 2006).

NVSS is a $1.4 \mathrm{GHz}$ continuum survey covering the entire sky north of $-40^{\circ}$ declination. The angular resolution is about $45^{\prime \prime}$, and the noise level in the images is about $0.5 \mathrm{mJy} / \mathrm{beam}$. A detailed description of the survey can be found in Condon et al. (1998). In this paper, we use images provided by the homepage of NVSS.

\section{Results}

We performed map observations toward 38 high-mass starforming regions with a size of $10^{\prime} \times 10^{\prime}$, and obtained 44 clumps. Figure B.1 shows the contour maps of $I_{\mathrm{CN}}\left(\int T_{A}^{*}(\mathrm{CN}(1-0)) d \mathrm{v}\right)$, $I_{\mathrm{HCN}}\left(\int T_{A}^{*}(\mathrm{HCN}(1-0)) d \mathrm{v}\right)$, and $I_{\mathrm{HNC}}\left(\int T_{A}^{*}(\mathrm{HNC}(1-0)) d \mathrm{v}\right)$ for each clump plotted on gray scale maps of $6(3.6) \mathrm{cm}$ and 20 $\mathrm{cm}$ continuum emission. Because we wished to study global line ratios of high-mass star-forming clumps, HMSCs, HMPOs, and UCHII regions that were not associated with molecular clumps were discarded. Table A.1 lists all HMSCs, HMPOs, and UCHII regions associated with molecular clumps and their evolutionary stages. Table A.2 lists all dense molecular clumps and information of VLA observations and results.

\footnotetext{
2 http://third.ucllnl.org/gps/index.html

3 http://www.cv.nrao.edu/nvss/

4 http://archive.nrao.edu/archive/e2earchivex.jsp
}

A key for studying variations of the global molecular line ratios is to identify the molecular clumps at different evolutionary stages. The clumps that contain HMSC, HMPO, and UCHII were named HMSC clump (HMSCC), HMPO clump (HMPOC), and UCHII clump (UCHIIC). For molecular clumps that contain several star-forming objects at different evolutionary stages, the stage of the clump is determined by the most evolved object. We suggest that the chemical conditions of clumps around HII regions, which are named HII clump (HIIC), are dominated by the HII region. Detailed information for each clump is given in Appendix D.

We finally identified 5 HMSCCs, 14 HMPOCs, 15 UCHIICs, and 10 HIICs. We averaged all detected CN(1-0), $\mathrm{HCN}(1-0)$, and $\mathrm{HNC}(1-0)$ emission from each clump to obtain the averaged molecular spectral lines (Fig. C.1), which were then fitted with a Gaussian to acquire their parameters. Tables A.3 to A.5 list the velocity $\left(V_{L S R}\right)$, full width at half maximum (FWHM) and peak intensity $\left(T_{A}^{*}\right)$ of the $\mathrm{CN}(1-0), \mathrm{HCN}(1-0)$ and $\mathrm{HNC}(1-0)$ for each clump. Table 2 gives the integrated intensities $I_{\mathrm{CN}}, I_{\mathrm{HCN}}$, and $I_{\mathrm{HNC}}$ and the line ratios $I_{\mathrm{CN}} / I_{\mathrm{HCN}}, I_{\mathrm{CN}} / I_{\mathrm{HNC}}$, and $I_{\mathrm{HCN}} / I_{\mathrm{HNC}}$. All transitions of the averaged CN(1-0) line from G22.35+0.41, G23.60+0.00A, G23.60+0.00B, and G52.940.59 , and $\mathrm{HCN}(1-0)$ line from D53.94-0.59 are very weak with signal-to-noise ratios between 1 and 3 . We did not fit them with a Gaussian, but just gave their integrated intensities in Table 2. Here $I_{\mathrm{CN}}$ integrated over two satellites, which are $\mathrm{CN} \mathrm{1-0} J=$ $3 / 2-1 / 2 F=3 / 2-1 / 2(113488.140 \mathrm{MHz})$ and $J=3 / 2-1 / 2 F=$ $5 / 2-3 / 2(113490.982 \mathrm{MHz}) . I_{\mathrm{HCN}}$ integrated over three satellites, which are HCN 1-0 $F=1-1(88630.4157 \mathrm{MHZ}), F=2-1$ $(88631.8473 \mathrm{MHz})$ and $F=0-1(88633.9360 \mathrm{MHz}) . I_{\mathrm{HNC}}$ integrated over three satellites, which are HNC 1-0 $F=0-1$ (90663.450 MHz), $F=2-1(90663.574 \mathrm{MHz})$ and $F=1-1$ (90663.656 MHz).

\section{Discussion}

\section{1. $I_{\mathrm{CN}} / I_{\mathrm{HCN}}, I_{\mathrm{CN}} / I_{\mathrm{HNC}}$ and $I_{\mathrm{HCN}} / I_{\mathrm{HNC}}$ for molecular clumps at different evolutionary stages}

Radio continuum emission at $6 \mathrm{~cm}$ (or $3.6 \mathrm{~cm}$ ) and $20 \mathrm{~cm}$ is free-free emission, which is produced by free electrons scattering off ions without being captured. Detection of radio freefree emission indicates a ionized hydrogen cloud (HII region). The latter requires abundance of ionized photons (UV photons), and this implies the existence of nearby $\mathrm{O} / \mathrm{B}$ stars. It is therefore natural to expect that these nearby stars will chemically alter the surrounding clumps (Schilke et al. 1992; Fuente et al. 1993), the shock fronts created by these stars as a result of mass loss phenomena are expected to modify the gas chemistry in the clumps. Furthermore, the photodissociation and photoionization of molecules by the UV radiation can drastically change the chemical composition in the regions where molecules are poorly shielded (Fuente et al. 1993; Caselli et al. 2011). However, such changes may not be detectable if the clump and the HII region traced by $6 \mathrm{~cm}$ or $20 \mathrm{~cm}$ continuum emission are at different distances along the line of sight.

This discussion suggests that if one clump is associated with $20 \mathrm{~cm}$ radio continuum emission, its chemistry may be strongly affected by nearby $\mathrm{O} / \mathrm{B}$ stars. It is therefore important to know whether there is a difference in the global molecular line ratios between the clumps that coincide with $20 \mathrm{~cm}$ continuum emission and the clumps that do not.

Figure 1 illustrates the global line ratios $I_{\mathrm{CN}} / I_{\mathrm{HCN}}, I_{\mathrm{CN}} / I_{\mathrm{HNC}}$, and $I_{\mathrm{HCN}} / I_{\mathrm{HNC}}$ for HMSCCs. The mean values of $I_{\mathrm{CN}} / I_{\mathrm{HCN}}$ for 
Table 2. Global line ratios for $\mathrm{CN}(1-0), \mathrm{HCN}(1-0)$, and $\mathrm{HNC}(1-0)$ for each clump.

\begin{tabular}{|c|c|c|c|c|c|c|c|c|}
\hline Source & $I_{\mathrm{CN}}$ & $I_{\mathrm{HCN}}$ & $I_{\mathrm{HNC}}$ & $\frac{I_{\mathrm{CN}}}{I_{\mathrm{HCN}}}$ & $\frac{I_{\mathrm{CN}}}{I_{\mathrm{HNC}}}$ & $\frac{I_{\mathrm{HCN}}}{I_{\mathrm{HNC}}}$ & Evolution stage & Note \\
\hline $\mathrm{G} 22.35+0.41$ & $0.37(0.06)$ & $1.52(0.04)$ & $0.34(0.05)$ & 0.25 & 1.09 & 4.41 & HMSCC & $\mathrm{Y}$ \\
\hline $\mathrm{G} 23.60+0.00 \mathrm{~A}$ & $0.11(0.11)$ & $0.29(0.05)$ & $0.77(0.05)$ & 0.37 & 0.14 & 0.38 & HMSCC & $\mathrm{N}$ \\
\hline $\mathrm{G} 23.60+0.00 \mathrm{~B}$ & $0.63(0.09)$ & $2.52(0.06)$ & $0.71(0.05)$ & 0.25 & 0.89 & 3.56 & UCHIIC & $\mathrm{Y}$ \\
\hline $\mathrm{G} 34.43+0.24 \mathrm{~A}$ & $0.67(0.05)$ & $1.49(0.05)$ & $1.44(0.04)$ & 0.45 & 0.46 & 1.03 & HMSCC & $\mathrm{N}$ \\
\hline G34.43+0.24B & $1.08(0.06)$ & $2.34(0.05)$ & $2.42(0.04)$ & 0.46 & 0.45 & 0.96 & HMPOC & $\mathrm{N}$ \\
\hline I18151-1208A & $2.00(0.07)$ & $3.71(0.04)$ & $1.28(0.04)$ & 0.54 & 1.56 & 2.91 & HMSCC & $\mathrm{Y}$ \\
\hline I18151-1208B & $1.92(0.07)$ & $4.23(0.04)$ & $1.16(0.04)$ & 0.46 & 1.66 & 3.65 & HMPOC & $\mathrm{Y}$ \\
\hline I18223-1243A & $0.88(0.04)$ & $1.84(0.04)$ & $1.06(0.03)$ & 0.48 & 0.83 & 1.74 & HMSCC & Y \\
\hline I18223-1243B & $1.44(0.06)$ & $2.18(0.04)$ & $1.18(0.04)$ & 0.66 & 1.22 & 1.85 & UCHIIC & $\mathrm{N}$ \\
\hline I18306-0835 & $0.51(0.08)$ & $1.67(0.07)$ & $0.87(0.05)$ & 0.31 & 0.59 & 1.92 & UCHIIC & $\mathrm{N}$ \\
\hline I18310-0825A & $0.17(0.05)$ & $1.06(0.05)$ & $0.70(0.03)$ & 0.16 & 0.24 & 1.50 & HMPOC & $\mathrm{Y}$ \\
\hline I18310-0825B & $0.25(0.07)$ & $0.90(0.07)$ & $0.77(0.04)$ & 0.27 & 0.32 & 1.17 & HMPOC & $\mathrm{Y}$ \\
\hline I18337-0743 & $0.37(0.08)$ & $1.15(0.09)$ & $1.26(0.08)$ & 0.33 & 0.30 & 0.91 & HMPOC & $\mathrm{Y}$ \\
\hline I18530+0215 & $0.63(0.06)$ & $1.40(0.04)$ & $1.15(0.02)$ & 0.45 & 0.55 & 1.22 & UCHIIC & $\mathrm{N}$ \\
\hline I05358+3543 & $1.21(0.06)$ & $3.93(0.05)$ & $2.02(0.05)$ & 0.31 & 0.60 & 1.95 & HMPOC & $\mathrm{N}$ \\
\hline I18445-0222 & $0.96(0.05)$ & $1.89(0.06)$ & $0.71(0.04)$ & 0.51 & 1.35 & 2.66 & UCHIIC & $\mathrm{Y}$ \\
\hline I18517+0437 & $1.22(0.06)$ & $3.22(0.04)$ & $1.07(0.03)$ & 0.38 & 1.13 & 3.00 & HMPOC & $\mathrm{Y}$ \\
\hline I20126+4104 & $2.03(0.09)$ & $4.46(0.04)$ & $1.86(0.04)$ & 0.45 & 1.09 & 2.40 & HMPOC & $\mathrm{Y}$ \\
\hline I20293+3952 & $1.15(0.06)$ & $2.06(0.06)$ & $1.54(0.02)$ & 0.56 & 0.75 & 1.34 & HMPOC & $\mathrm{N}$ \\
\hline $\mathrm{I} 20343+4129$ & $1.78(0.06)$ & $3.05(0.16)$ & $1.18(0.03)$ & 0.58 & 1.50 & 2.58 & HMPOC & $\mathrm{Y}$ \\
\hline I22134+5834 & $0.64(0.05)$ & $0.92(0.02)$ & $0.37(0.03)$ & 0.70 & 1.74 & 2.48 & HMPOC & $\mathrm{Y}$ \\
\hline $\mathrm{I} 22551+6221$ & $0.85(0.05)$ & $1.18(0.04)$ & $0.43(0.03)$ & 0.73 & 2.01 & 2.76 & HIIC & $\mathrm{Y}$ \\
\hline $\mathrm{I} 22570+5912$ & $0.58(0.03)$ & $1.08(0.04)$ & $0.30(0.03)$ & 0.54 & 1.92 & 3.56 & HIIC & Y \\
\hline I23033+5951 & $0.67(0.03)$ & $1.69(0.05)$ & $1.00(0.04)$ & 0.40 & 0.67 & 1.69 & HMPOC & $\mathrm{Y}$ \\
\hline I23139+5939 & $0.54(0.03)$ & $1.17(0.04)$ & $0.40(0.04)$ & 0.46 & 1.36 & 2.95 & HMPOC & $\mathrm{Y}$ \\
\hline I01202+6133 & $0.85(0.04)$ & $2.20(0.06)$ & $0.93(0.02)$ & 0.39 & 0.91 & 2.36 & HMPOC & $\mathrm{Y}$ \\
\hline $\mathrm{I} 03035+5819$ & $1.16(0.06)$ & $2.04(0.06)$ & $0.79(0.04)$ & 0.57 & 1.45 & 2.57 & UCHIIC & $\mathrm{N}$ \\
\hline I19078+0901 & $3.20(0.10)$ & $5.94(0.06)$ & $1.57(0.04)$ & 0.54 & 2.03 & 3.77 & UCHIIC & $\mathrm{N}$ \\
\hline I19097+0847 & $1.29(0.05)$ & $1.80(0.02)$ & $1.04(0.05)$ & 0.72 & 1.24 & 1.72 & UCHIIC & $\mathrm{N}$ \\
\hline I19111+1048A & $2.18(0.06)$ & $4.80(0.09)$ & $1.55(0.05)$ & 0.45 & 1.40 & 3.09 & UCHIIC & $\mathrm{N}$ \\
\hline I19111+1048B & $1.24(0.08)$ & $2.23(0.09)$ & $0.98(0.06)$ & 0.56 & 1.27 & 2.29 & UCHIIC & $\mathrm{N}$ \\
\hline I19442+2427 & $4.24(0.10)$ & $7.66(0.04)$ & $2.53(0.04)$ & 0.55 & 1.68 & 3.03 & UCHIIC & $\mathrm{N}$ \\
\hline $\mathrm{I} 20081+3122$ & $0.69(0.11)$ & $3.41(0.05)$ & $1.70(0.03)$ & 0.20 & 0.41 & 2.00 & UCHIIC & $\mathrm{N}$ \\
\hline I23116+6111 & $2.95(0.07)$ & $9.39(0.03)$ & $3.63(0.04)$ & 0.31 & 0.81 & 2.59 & HIIC & $\mathrm{Y}$ \\
\hline $\mathrm{I} 23133+6050$ & $1.13(0.08)$ & $3.39(0.06)$ & $0.94(0.04)$ & 0.33 & 1.21 & 3.62 & UCHIIC & $\mathrm{N}$ \\
\hline I $23138+5945$ & $1.70(0.05)$ & $3.24(0.04)$ & $0.99(0.04)$ & 0.52 & 1.71 & 3.27 & UCHIIC & $\mathrm{N}$ \\
\hline U49.49-0.37 & $9.38(0.15)$ & $15.59(0.09)$ & $9.58(0.10)$ & 0.60 & 0.98 & 1.63 & UCHIIC & $\mathrm{N}$ \\
\hline C41.1-0.21 & $0.51(0.03)$ & $1.32(0.05)$ & $0.47(0.03)$ & 0.38 & 1.08 & 2.84 & HIIC & $\mathrm{Y}$ \\
\hline C48.93-0.29 & $2.39(0.04)$ & $4.03(0.02)$ & $1.25(0.04)$ & 0.59 & 1.91 & 3.21 & HIIC & $\mathrm{Y}$ \\
\hline C49.00-0.30 & $3.66(0.06)$ & $5.67(0.04)$ & $2.03(0.04)$ & 0.65 & 1.80 & 2.80 & HIIC & $\mathrm{Y}$ \\
\hline C49.38-0.30 & $3.46(0.06)$ & $4.45(0.06)$ & $2.21(0.08)$ & 0.78 & 1.56 & 2.01 & HIIC & $\mathrm{Y}$ \\
\hline C51.36-0.00 & $0.96(0.03)$ & $1.54(0.02)$ & $0.94(0.03)$ & 0.62 & 1.02 & 1.63 & HIIC & $\mathrm{Y}$ \\
\hline C52.94-0.59 & $0.29(0.04)$ & $0.66(0.05)$ & $0.12(0.03)$ & 0.44 & 2.53 & 5.71 & HIIC & $\mathrm{Y}$ \\
\hline D53.64+0.24 & $0.25(0.03)$ & $0.50(0.04)$ & $0.23(0.03)$ & 0.51 & 1.12 & 2.20 & HIIC & $\mathrm{Y}$ \\
\hline
\end{tabular}

Notes. From left to right, the columns are (1) source name; (2) integrated intensity of CN; (3) integrated intensity of HCN; (4) integrated intensity of $\mathrm{HNC}$; (5) the integrated intensity ratios $I_{\mathrm{CN}} / I_{\mathrm{HCN}} ;(6)$ the integrated intensity ratios $I_{\mathrm{CN}} / I_{\mathrm{HNC}} ;(7)$ the integrated intensity ratios $I_{\mathrm{HCN}} / I_{\mathrm{HNC}} ;(8)$ the evolutionary stage of the source; (9) $\mathrm{N}$ signifies those sources that are not coincident with $20 \mathrm{~cm}$ continuum emission, and $\mathrm{Y}$ indicates sources that coincide with the $20 \mathrm{~cm}$ continuum emission.

HMSCCs coincide with $20 \mathrm{~cm}$ continuum emission, and those that do not are $0.41 \pm 0.04$ and $0.42 \pm 0.12$. However, the values of $I_{\mathrm{CN}} / I_{\mathrm{HNC}}$ for HMSCCs that coincide with $20 \mathrm{~cm}$ continuum emission are much higher than those for HMSCCs that do not coincide with $20 \mathrm{~cm}$ continuum emission, and their mean values are $1.16 \pm 0.3$ and $0.30 \pm 0.16$. This is the same for $I_{\mathrm{HCN}} / I_{\mathrm{HNC}}$, with mean values of $3.02 \pm 1.09$ and $0.71 \pm 0.32$. For HMSCCs that coincide with $20 \mathrm{~cm}$ continuum emission, this indicates a true connection between the respective clumps and the associated emission from the ionized gas, and the chemistry of the gas seems to change due to the existence of some nearby O/B stars. $I_{\mathrm{CN}} / I_{\mathrm{HNC}}$ and $I_{\mathrm{HCN}} / I_{\mathrm{HNC}}$ for HMSCCs are sensitive to the appearance of $20 \mathrm{~cm}$ continuum emission.
Figure 2 shows the global line ratios $I_{\mathrm{CN}} / I_{\mathrm{HCN}}, I_{\mathrm{CN}} / I_{\mathrm{HNC}}$, and $I_{\mathrm{HCN}} / I_{\mathrm{HNC}}$ for HMPOCs. The $I_{\mathrm{CN}} / I_{\mathrm{HCN}}$ values for HMPOCs that coincide with $20 \mathrm{~cm}$ continuum emission are almost the same as those of HMPOCs that do not coincide with $20 \mathrm{~cm}$ continuum emission, and their mean values are $0.42 \pm 0.14$ and $0.44 \pm 0.11$. The ratios of $I_{\mathrm{CN}} / I_{\mathrm{HCN}}$ for HMPOCs are similar to that of HMSCCs, and both are insensitive to the 20 $\mathrm{cm}$ continuum emission. Most $I_{\mathrm{CN}} / I_{\mathrm{HNC}}$ values for HMPOCs that coincide with $20 \mathrm{~cm}$ continuum emission are higher than those for HMPOCs that do not coincide with $20 \mathrm{~cm}$ continuum emission, and their corresponding mean values are $0.98 \pm 0.52$ and $0.60 \pm 0.12$. The mean values of $I_{\mathrm{HCN}} / I_{\mathrm{HNC}}$ for HMPOCs that coincide and HMPOCs that do not coincide with $20 \mathrm{~cm}$ 
Table 3. Global line ratios for $\mathrm{CN}(1-0), \mathrm{HCN}(1-0), \mathrm{HNC}(1-0)$, and $\mathrm{HCO}^{+}(1-0)$ for ten galaxies.

\begin{tabular}{lcclccc}
\hline \hline Source & $\frac{I_{\mathrm{CN}}}{I_{\mathrm{HCN}}}$ & $\frac{I_{\mathrm{CN}}}{I_{\mathrm{HNC}}}$ & $\frac{I_{\mathrm{HCN}}}{I_{\mathrm{HNC}}}$ & $\frac{I_{\mathrm{HCO}}}{I_{\mathrm{HCN}}}$ & $\log \left(\frac{L_{\mathrm{IR}}}{L_{\odot}}\right)$ & Type \\
\hline NGC 7771 & 0.817 & 1.39 & 1.7 & 0.912 & 11.24 & $\mathrm{~L}$ \\
NGC 3079 & 1.052 & 3.964 & 3.766 & 1.124 & 10.65 & $\mathrm{~A}$ \\
NGC 3556 & 1.111 & 3.75 & 3.375 & 1.574 & 10.00 & $\mathrm{~S}$ \\
NGC 6240 & 1.326 & 6.727 & 5.07 & 1.627 & 11.69 & $\mathrm{~L}$ \\
UGC 2866 & 1.347 & 2.64 & 1.96 & 1.462 & 10.69 & $\mathrm{~S}$ \\
NGC 660 & 1.396 & 2.68 & 1.92 & 1.042 & 10.40 & $\mathrm{~S}$ \\
NGC 7469 & 1.421 & 2.545 & 1.791 & 1.137 & 11.41 & $\mathrm{~A}$ \\
NGC 1614 & 1.658 & 5.458 & 3.29 & 1.898 & 11.43 & $\mathrm{~S}$ \\
UGC 5101 & 1.682 & 2.055 & 1.22 & 0.364 & 11.87 & $\mathrm{~A}, \mathrm{~L}$ \\
NGC 4194 & 2.510 & 7.866 & 1.88 & 1.319 & 10.93 & $\mathrm{~S}$ \\
\hline
\end{tabular}

Notes. From left to right, the columns are (1) source name; (2) the integrated intensity ratios $I_{\mathrm{CN}} / I_{\mathrm{HCN}}$; (3) the integrated intensity ratios $I_{\mathrm{CN}} / I_{\mathrm{HNC}} ;(4)$ the integrated intensity ratios $I_{\mathrm{HCN}} / I_{\mathrm{HNC}} ;(5)$ the integrated intensity ratios $I_{\mathrm{HCO}^{+}} / I_{\mathrm{HCN}}$. (6) the infrared luminosity of the galaxy; (7) the type of the galaxy where L, A, and S represent a luminous infrared galaxy, an active galactic nucleus, and a starburst galaxy. The data shown here come from Costagliola et al. (2011).
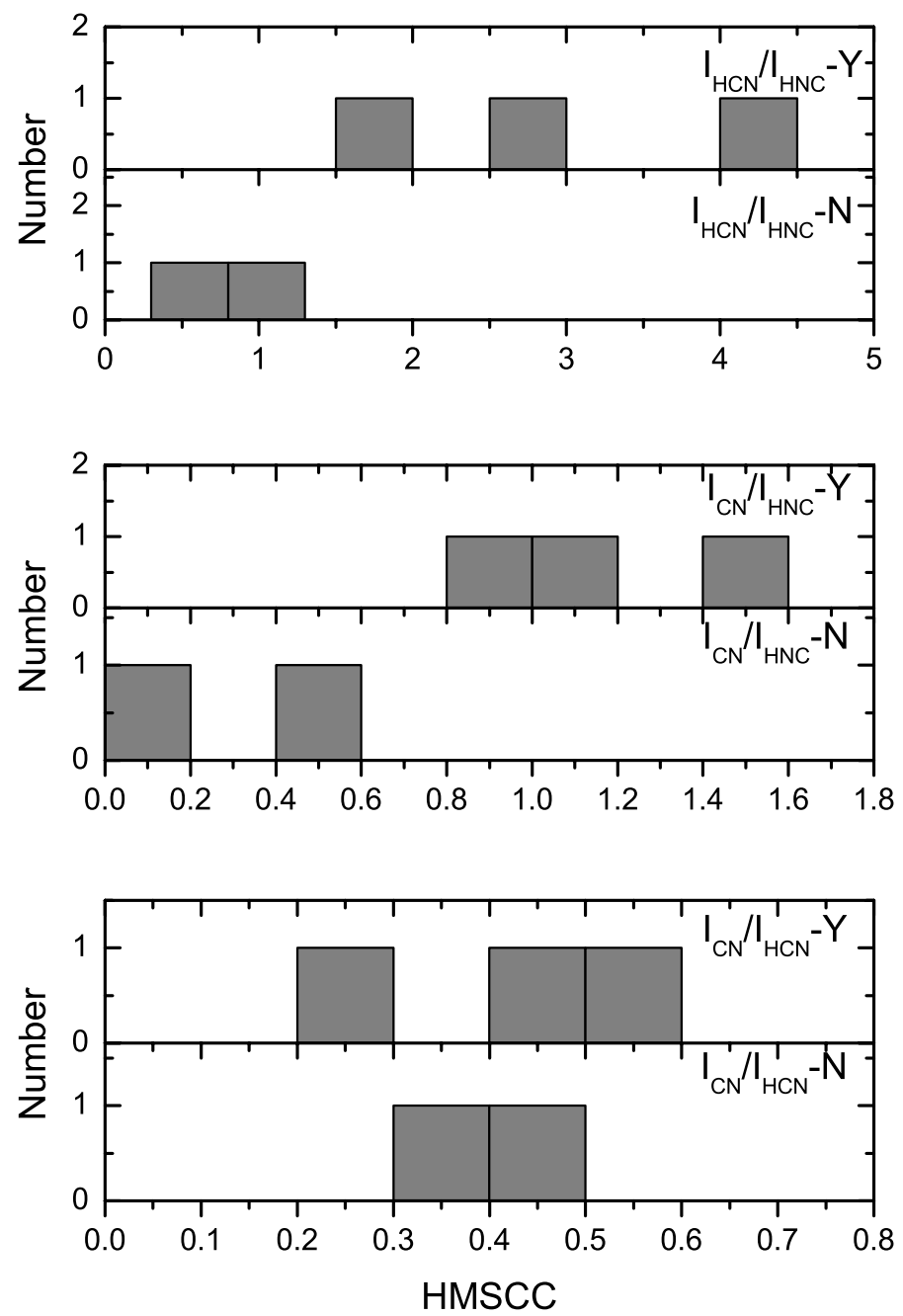

Fig. 1. Global line ratios $I_{\mathrm{HCN}} / I_{\mathrm{HNC}}, I_{\mathrm{CN}} / I_{\mathrm{HNC}}$ and $I_{\mathrm{CN}} / I_{\mathrm{HCN}}$ for highmass starless clumps that coincide with $20 \mathrm{~cm}$ continuum emission (denoted by $I_{\mathrm{HCN}} / I_{\mathrm{HNC}}-\mathrm{Y}, I_{\mathrm{CN}} / I_{\mathrm{HNC}}-\mathrm{Y}$ and $\left.I_{\mathrm{CN}} / I_{\mathrm{HCN}}-\mathrm{Y}\right)$ and those highmass starless core clumps that do not coincide with $20 \mathrm{~cm}$ continuum emission (denoted by $I_{\mathrm{HCN}} / I_{\mathrm{HNC}}-\mathrm{N}, I_{\mathrm{CN}} / I_{\mathrm{HNC}}-\mathrm{N}$ and $I_{\mathrm{CN}} / I_{\mathrm{HCN}}-\mathrm{N}$ ).
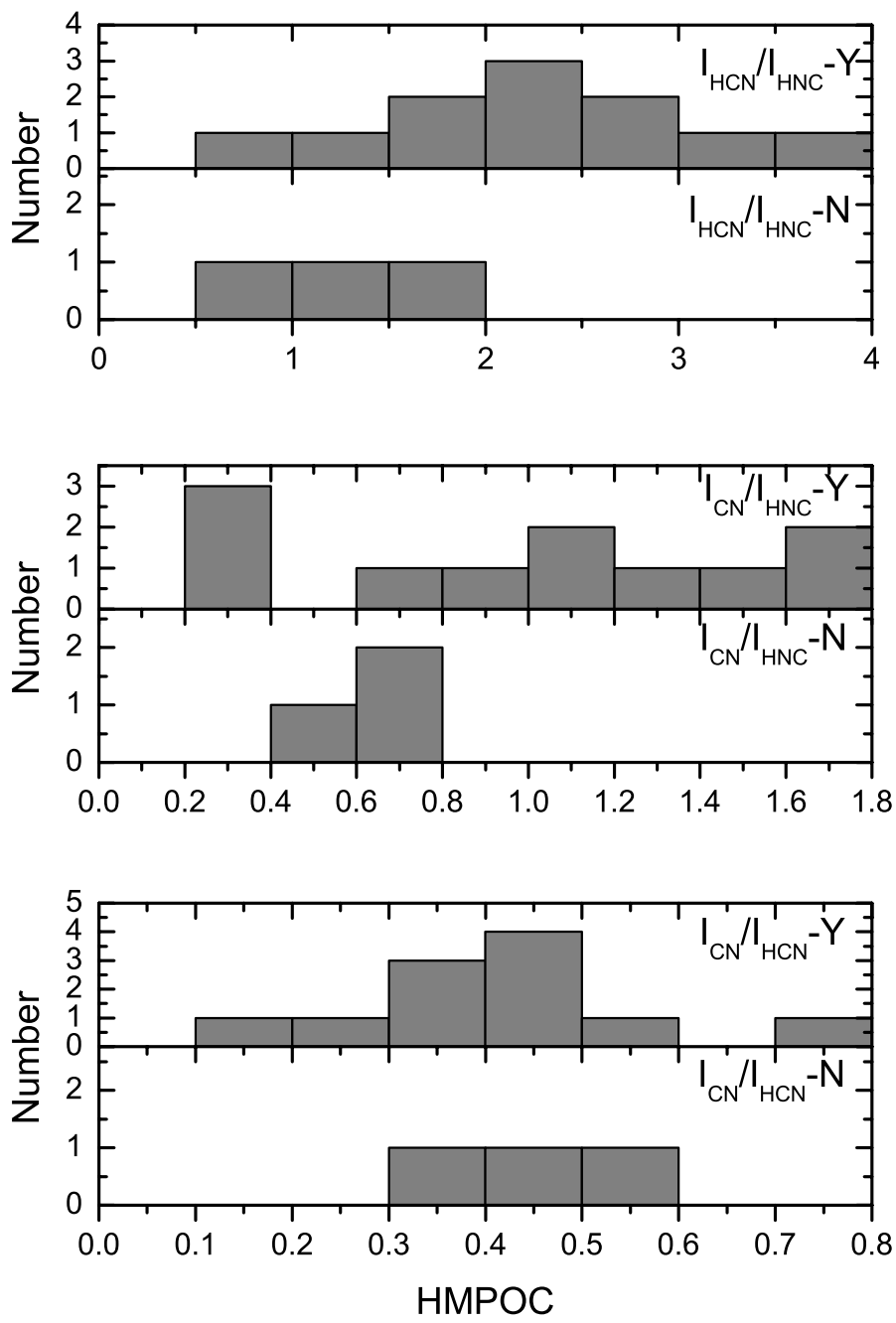

Fig. 2. Global line ratios $I_{\mathrm{HCN}} / I_{\mathrm{HNC}}, I_{\mathrm{CN}} / I_{\mathrm{HNC}}$ and $I_{\mathrm{CN}} / I_{\mathrm{HCN}}$ for highmass protostar clumps that coincide with $20 \mathrm{~cm}$ continuum emission (denoted by $I_{\mathrm{HCN}} / I_{\mathrm{HNC}}-\mathrm{Y}, I_{\mathrm{CN}} / I_{\mathrm{HNC}}-\mathrm{Y}$ and $I_{\mathrm{CN}} / I_{\mathrm{HCN}}-\mathrm{Y}$ ) and those highmass protostar clumps that do not coincide with $20 \mathrm{~cm}$ continuum emission (denoted by $I_{\mathrm{HCN}} / I_{\mathrm{HNC}}-\mathrm{N}, I_{\mathrm{CN}} / I_{\mathrm{HNC}}-\mathrm{N}$ and $I_{\mathrm{CN}} / I_{\mathrm{HCN}}-\mathrm{N}$ ).

continuum emission are $2.24 \pm 0.77$ and $1.42 \pm 0.41$. It seems that the relative variations caused by nearby $\mathrm{O} / \mathrm{B}$ stars are not as strong as the variations found in HMSCCs. This may be ascribed to two reasons. One is that star formation activities in the HMPOC play an important role in its chemical conditions. Another is that there may be no association between some HMPOCs and the $20 \mathrm{~cm}$ continuum emission, which are merely coinciding along the line of sight.

HMPOCs I18310-0825A, I18310-0825B, I18337-0743, and I23033+5951 coincide with $20 \mathrm{~cm}$ continuum emission, but their line ratios $I_{\mathrm{CN}} / I_{\mathrm{HNC}}$ and $I_{\mathrm{HCN}} / I_{\mathrm{HNC}}$ are lower than or approximate to those of HMPOCs that do not coincide with $20 \mathrm{~cm}$ continuum emission. Hence we tentatively assume that these four HMPOs are not associated with $20 \mathrm{~cm}$ continuum emission; they just coincide along the line of sight. Then, $I_{\mathrm{CN}} / I_{\mathrm{HCN}}, I_{\mathrm{CN}} / I_{\mathrm{HNC}}$, and $I_{\mathrm{HCN}} / I_{\mathrm{HNC}}$ for HMPOCs associated with $20 \mathrm{~cm}$ continuum emission are $0.49 \pm 0.1,1.34 \pm 0.54$, and $2.77 \pm 0.43$, respectively, and the corresponding mean values for HMPOCs that are not associated with $20 \mathrm{~cm}$ continuum emission are $0.36 \pm 0.12$, $0.48 \pm 0.18$ and $1.36 \pm 0.35$, respectively. This suggests that star formation activities in HMPOCs play a small role in their surface chemistry; $I_{\mathrm{CN}} / I_{\mathrm{HNC}}$ and $I_{\mathrm{HCN}} / I_{\mathrm{HNC}}$ of HMPOCs are also sensitive to the appearance of $20 \mathrm{~cm}$ continuum emission. 

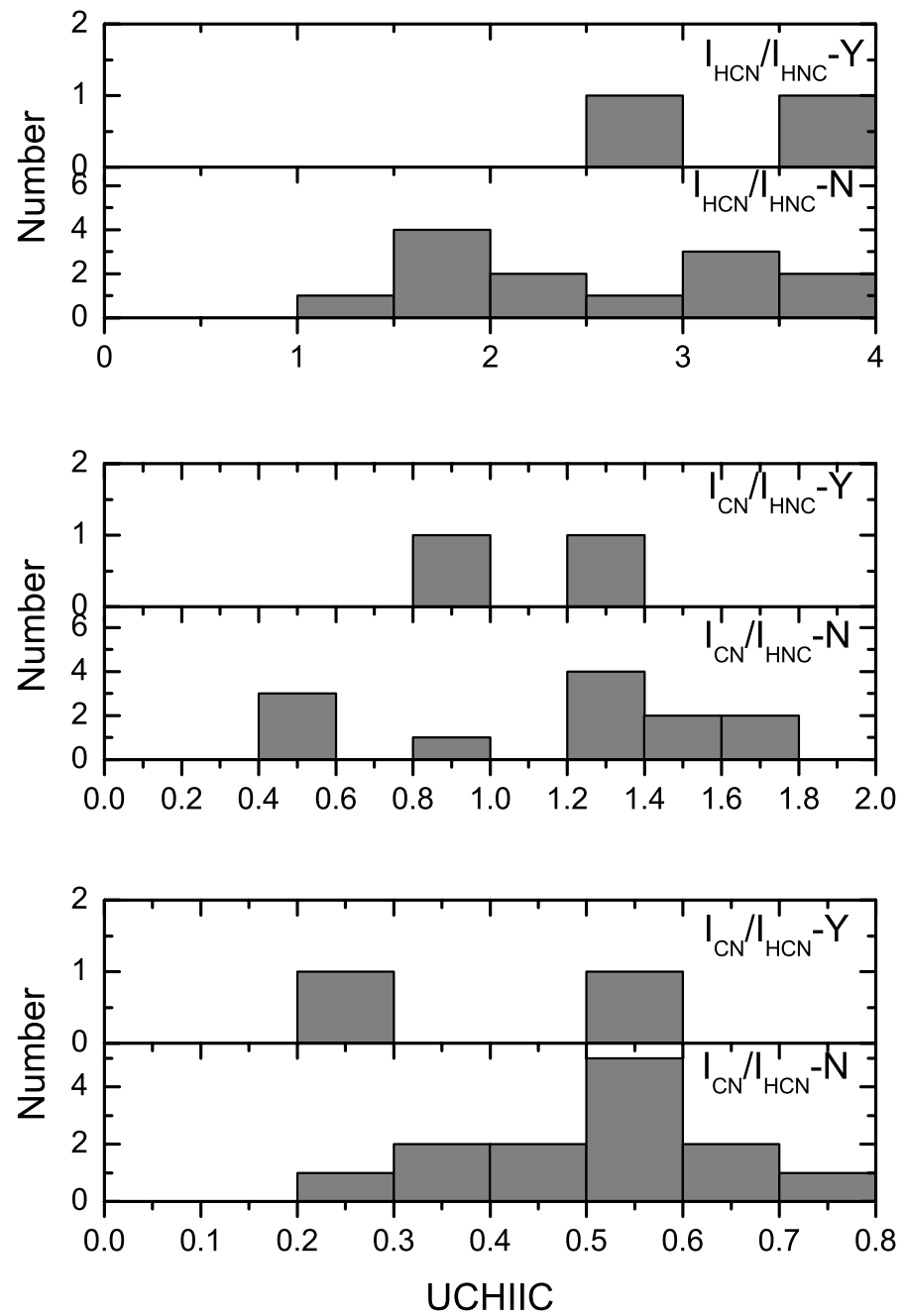

Fig. 3. Global line ratios $I_{\mathrm{HCN}} / I_{\mathrm{HNC}}, I_{\mathrm{CN}} / I_{\mathrm{HNC}}$ and $I_{\mathrm{CN}} / I_{\mathrm{HCN}}$ for UCHII region clumps that coincide with $20 \mathrm{~cm}$ continuum emission (denoted by $I_{\mathrm{HCN}} / I_{\mathrm{HNC}}-\mathrm{Y}, I_{\mathrm{CN}} / I_{\mathrm{HNC}}-\mathrm{Y}$ and $\left.I_{\mathrm{CN}} / I_{\mathrm{HCN}}-\mathrm{Y}\right)$ and those UCHII region clumps that do not coincide with $20 \mathrm{~cm}$ continuum emission (denoted by $I_{\mathrm{HCN}} / I_{\mathrm{HNC}}-\mathrm{N}, I_{\mathrm{CN}} / I_{\mathrm{HNC}}-\mathrm{N}$ and $\left.I_{\mathrm{CN}} / I_{\mathrm{HCN}}-\mathrm{N}\right)$.

UCHIICs usually show strong radio emission at $3.6(6) \mathrm{cm}$ and $20 \mathrm{~cm}$ wavelength. High-mass star formation processes may have ended at this stage, and newly formed stars will strongly affect the physical and chemical conditions of the parental clump. The global line ratios $I_{\mathrm{CN}} / I_{\mathrm{HCN}}, I_{\mathrm{CN}} / I_{\mathrm{HNC}}$, and $I_{\mathrm{HCN}} / I_{\mathrm{HNC}}$ of UCHIICs that coincide with $20 \mathrm{~cm}$ continuum emission are similar to those of UCHIICs that do not (see Fig. 3), and the mean values are $0.38 \pm 0.13$ and $0.5 \pm 0.14$ for $I_{\mathrm{CN}} / I_{\mathrm{HCN}}, 1.12 \pm 0.23$ and $1.21 \pm 0.46$ for $I_{\mathrm{CN}} / I_{\mathrm{HNC}}$, and $3.11 \pm 0.45$ and $2.46 \pm 0.79$ for $I_{\mathrm{HCN}} / I_{\mathrm{HNC}}$. This suggests that star formation activities in UCHIICs play an important role in their physical and chemical conditions. $I_{\mathrm{CN}} / I_{\mathrm{HNC}}$ and $I_{\mathrm{HCN}} / I_{\mathrm{HNC}}$ for UCHIICs are not sensitive to the appearance of $20 \mathrm{~cm}$ continuum emission.

HIICs are molecular clouds surrounding large HII regions (see Fig. B.1), where high-mass stars produce large amounts of ionizing photons with energies above $13.6 \mathrm{eV}$ that are capable of ionizing hydrogen in the vicinity. HIICs in our sample all coincide with strong and extended 20 (or 6) cm continuum emission. The physical and chemical conditions of HIICs are inevitably affected by the HII region. The mean values of $I_{\mathrm{CN}} / I_{\mathrm{HCN}}, I_{\mathrm{CN}} / I_{\mathrm{HNC}}$, and $I_{\mathrm{HCN}} / I_{\mathrm{HNC}}$ for HIICs are $0.56 \pm 0.14$,
$1.58 \pm 0.52$ and $2.93 \pm 1.07$, respectively, and are higher than the corresponding values for UCHIICs.

In summary, the global line ratios $I_{\mathrm{CN}} / I_{\mathrm{HNC}}$ and $I_{\mathrm{HCN}} / I_{\mathrm{HNC}}$ for HMSCCs and HMPOCs that are associate with $20 \mathrm{~cm}$ continuum emission are much higher than those for HMSCCs and HMPOCs that are not associated with $20 \mathrm{~cm}$ continuum emission. This strongly confirms that the $20 \mathrm{~cm}$ continuum emission indicates the existence of some O/B stars, whose stellar wind and UV photons affect the surface chemistry of the nearby clumps (Caselli et al. 2011). Star formation activities proceeding in HMSCCs and HMPOCs seem to contribute little to their surface chemistry. The $I_{\mathrm{CN}} / I_{\mathrm{HNC}}$ and $I_{\mathrm{HCN}} / I_{\mathrm{HNC}}$ of UCHIICs are mainly determined by their internal star formation activities.

\subsection{Variations of the line ratio in high-mass star-forming regions}

Since the global ratios $I_{\mathrm{CN}} / I_{\mathrm{HNC}}$ and $I_{\mathrm{HCN}} / I_{\mathrm{HNC}}$ for HMSCCs and HMPOCs are sensitive to the $20 \mathrm{~cm}$ continuum emission that is associated with them, we have to select the HMSCCs and HMPOCs that are not associated with external $20 \mathrm{~cm}$ continuum emission to study whether variations in the line ratios $I_{\mathrm{CN}} / I_{\mathrm{HNC}}$ and $I_{\mathrm{HCN}} / I_{\mathrm{HNC}}$ trace the evolution of high-mass star-forming regions. Because UCHIICs and HIICs are not sensitive to the $20 \mathrm{~cm}$ continuum emission associated with them, all of them are used in the analysis. The mean $I_{\mathrm{CN}} / I_{\mathrm{HCN}}$ values for HMSCCs, HMPOCs, UCHIICs, and HIICs are $0.41 \pm 0.04,0.36 \pm 0.12$, $0.48 \pm 0.14$, and $0.56 \pm 0.14$, respectively, suggesting that the ratio is not sensitive to the evolution of high-mass star-forming regions. The mean $I_{\mathrm{CN}} / I_{\mathrm{HNC}}$ values for HMSCCs, HMPOCs, UCHIICs, and HIICs are $0.3 \pm 0.16,0.48 \pm 0.18,1.20 \pm 0.44$, and $1.58 \pm 0.52$, respectively. The mean $I_{\mathrm{HCN}} / I_{\mathrm{HNC}}$ values for HMSCCs, HMPOCs, UCHIICs, and HIICs are $0.71 \pm 0.32$, $1.36 \pm 0.35,2.55 \pm 0.79$, and $2.93 \pm 1.07$, respectively. Both $I_{\mathrm{CN}} / I_{\mathrm{HNC}}$ and $I_{\mathrm{HCN}} / I_{\mathrm{HNC}}$ increase with the age of star-forming regions, and this suggests that they are promising to be good indicators of the high-mass star formation sequence. In contrast to the result of Hoq et al. (2013), our result for $I_{\mathrm{HCN}} / I_{\mathrm{HNC}}$ displays a much more obviously increasing trend with proceeding evolution of high-mass star-forming regions.

However, we note that $I_{\mathrm{CN}} / I_{\mathrm{HNC}}$ values for HMSCCs are similar to those for HMPOCs, but much lower than those for UCHIICs and HIICs, the latter two having similar $I_{\mathrm{CN}} / I_{\mathrm{HNC}}$. The variation of $I_{\mathrm{HCN}} / I_{\mathrm{HNC}}$ is similar to that of $I_{\mathrm{CN}} / I_{\mathrm{HNC}}$. It is therefore reasonable to divide our sources into two types. Type 1 includes HMSCCs and HMPOCs that are not associated with external $20 \mathrm{~cm}$ continuum emission. Type 2 includes all UCHIICs and HIICs. We can then expect the global line ratios $I_{\mathrm{CN}} / I_{\mathrm{HNC}}$ and $I_{\mathrm{HCN}} / I_{\mathrm{HNC}}$ to trace the evolution from type 1 to type 2 sources well.

Figure 4 shows the distribution of the global line ratios $I_{\mathrm{CN}} / I_{\mathrm{HNC}}$ and $I_{\mathrm{HCN}} / I_{\mathrm{HNC}}$ for type 1 and type 2 sources. The mean values of $I_{\mathrm{CN}} / I_{\mathrm{HNC}}$ and $I_{\mathrm{HCN}} / I_{\mathrm{HNC}}$ are $0.44 \pm 0.19$ and $1.21 \pm 0.44$ for type 1 sources, and $1.35 \pm 0.5$ and $2.70 \pm 0.93$ for type 2 sources. $I_{\mathrm{CN}} / I_{\mathrm{HNC}}$ and $I_{\mathrm{HCN}} / I_{\mathrm{HNC}}$ display an obvious trend of increase from type 1 to type 2 sources, but there is still some overlap between the type 1 and 2 sources. This may be because high-mass star-forming regions evolve continuously from HMSCs to HMPOs, to UCHII, and finally to HII regions, and there is some overlap between the end of one evolutionary stage and the beginning of the next evolutionary stage.

Figure 5 shows a diagram of $I_{\mathrm{CN}} / I_{\mathrm{HNC}}$ versus $I_{\mathrm{HCN}} / I_{\mathrm{HNC}}$, which indicates that most type 1 and type 2 sources can be distinguished from each other. Hence the global line ratios $I_{\mathrm{CN}} / I_{\mathrm{HNC}}$ 
X. H. Han et al.: A possible indicator for high-mass star formation sequence
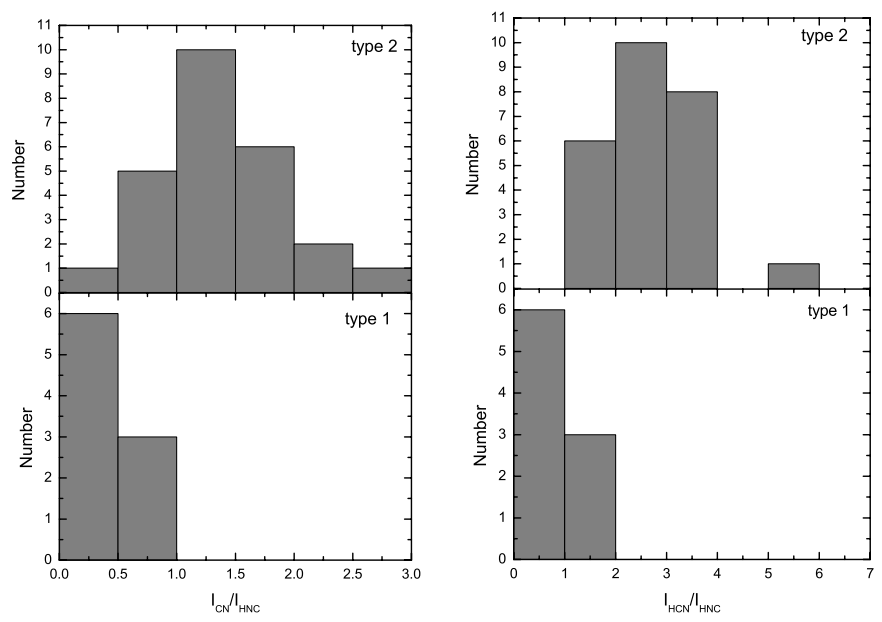

Fig. 4. Global line ratios $I_{\mathrm{HCN}} / I_{\mathrm{HNC}}$ and $I_{\mathrm{CN}} / I_{\mathrm{HNC}}$ for type 1 and 2 sources, which show a clear trend of increase from type 1 to type 2 sources.

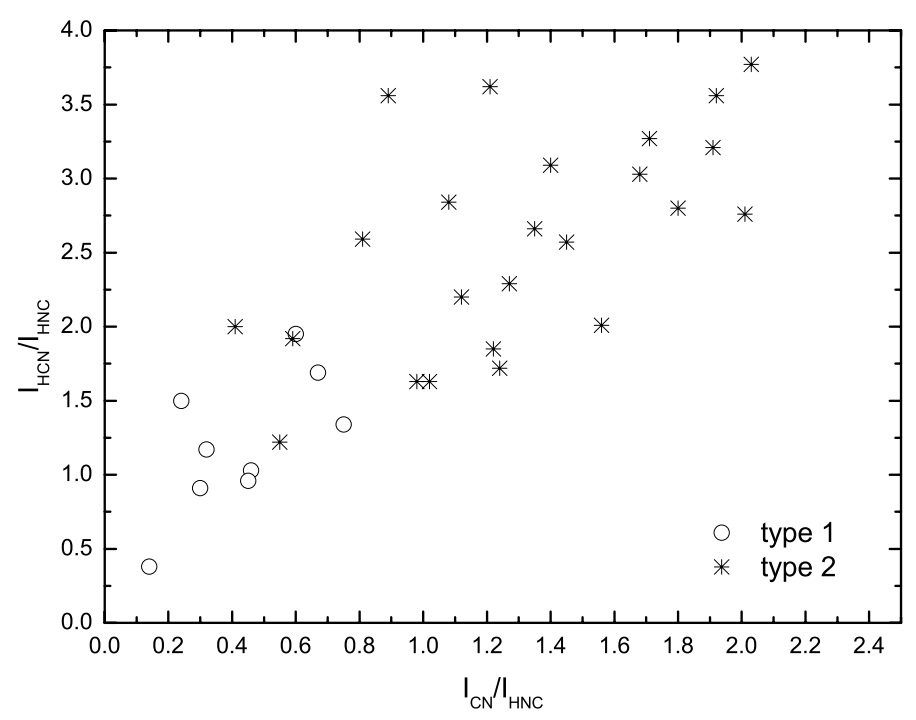

Fig. 5. Plot of $I_{\mathrm{HCN}} / I_{\mathrm{HNC}}$ versus $I_{\mathrm{CN}} / I_{\mathrm{HNC}}$ showing that type 1 and 2 sources can be distinguished from each other. Circles and asterisks represent type 1 and type 2 sources.

and $I_{\mathrm{HCN}} / I_{\mathrm{HNC}}$ appear to be good tracers for the evolution of high-mass star-forming regions.

\subsection{Distributions of $C N(1-0), H C N(1-0)$, and $H N C(1-0)$ for the clumps}

Figure B.1 suggests that $\mathrm{CN}(1-0), \mathrm{HCN}(1-0)$, and $\mathrm{HNC}(1-0)$ have a similar distribution for most dense clumps. However, this is not true when the dense clump is strongly affected by $\mathrm{O} / \mathrm{B}$ stars on one side or where the clump is diffuse or partly diffuse. Figure 6 displays the distributions of $\mathrm{CN}(1-0), \mathrm{HCN}(1-0)$, and $\mathrm{HNC}(1-0)$ for four representative clumps.

For the HMPOC I05358+3543, the distributions of CN(1-0), $\mathrm{HCN}(1-0)$, and $\mathrm{HNC}(1-0)$ are similar. There is no 6 or $20 \mathrm{~cm}$ continuum emission nearby the clump. However, for the HMPOC I20343+4129, the distribution of $\mathrm{CN}(1-0)$ is similar to that of $\mathrm{HCN}(1-0)$, but is different from that of $\mathrm{HNC}(1-0)$. $\mathrm{CN}(1-0)$ and $\mathrm{HCN}(1-0)$ emission extends farther to the northeast than $\mathrm{HNC}(1-0)$ emission does. The fact that the clump partly coincides with $20 \mathrm{~cm}$ radio continuum emission indicates that $\mathrm{O} / \mathrm{B}$ stars may appear to the northeast of the clump; the differences between distributions of $\mathrm{CN}(1-0), \mathrm{HCN}(1-0)$, and $\mathrm{HNC}(1-0)$ may be ascribed to the influence of nearby $\mathrm{O} / \mathrm{B}$ stars (de Jong 1977; Hollenbach 1988). For HMPOC I18517+0437, $\mathrm{CN}(1-0), \mathrm{HCN}(1-0)$, and $\mathrm{HNC}(1-0)$ have similar distributions in the dense part of the clump. However, relatively strong and extended $\mathrm{CN}(1-0)$ emission was detected to the west and north of the clump where the gas is diffuse. This may be due to the low optical depth and enhanced UV radiation. G22.35+0.41 has diffuse clumps, where $\mathrm{HCN}(1-0)$ emission is relatively strong, $\mathrm{HNC}(1-0)$ and $\mathrm{CN}(1-0)$ are marginally detected. The distributions of $\mathrm{CN}(1-0), \mathrm{HCN}(1-0)$, and $\mathrm{HNC}(1-0)$ are different from each other.

In summary, $\mathrm{CN}(1-0), \mathrm{HCN}(1-0)$, and $\mathrm{HNC}(1-0)$ have similar distributions for the dense clumps without being affected by environment. $\mathrm{CN}(1-0)$ and $\mathrm{HCN}(1-0)$ have a similar distribution for the dense clumps exposed to strong UV radiation. Furthermore, $\mathrm{CN}(1-0), \mathrm{HCN}(1-0)$, and $\mathrm{HNC}(1-0)$ tend to have different distributions for the diffuse clump or for the diffuse part of the clump.

\subsection{Global properties of the ISM in external galaxies}

Our results suggest that the global line ratios between $\mathrm{CN}(1-0)$, $\mathrm{HCN}(1-0)$, and $\mathrm{HNC}(1-0)$ can be used to distinguish dense cold clumps from dense warm clumps and clumps associated with UCHII regions. Because external galaxies are far away from us, single-dish observations toward them usually cover a huge amounts of ISM, we thus obtain the global properties of the ISM. Taking into account that the global properties of the ISM in the galaxies may be determined by warm dense gas or PDRs or X-ray dominated region (XDRs; Aalto et al. 2007; Aalto 2013), we may use the global line ratios between $\mathrm{CN}(1-0), \operatorname{HCN}(1-0)$, and $\mathrm{HNC}(1-0)$ to distinguish them and to study the evolution of galaxies.

Fortunately, Costagliola et al. (2011) published their multimolecular line survey data for 24 nearby galaxies, which includes $\mathrm{CN}(1-0), \mathrm{HCN}(1-0), \mathrm{HNC}(1-0), \mathrm{HCO}^{+}(1-0)$, and many other lines (see their Table E.1). CN(1-0), HCN(1-0), HNC(1$0)$, and $\mathrm{HCO}^{+}(1-0)$ were detected in ten galaxies; we calculated their line ratios (see Table 3 ). Since $\mathrm{CN}$ is sensitive to the UV and $\mathrm{X}$-ray radiation, the abundance enhancement of $\mathrm{CN}$ over $\mathrm{HCN}$ is greater in XDR (factors 40 to 1000) than in PDR (factors 0.5 to 2) (Lepp \& Dalgarno 1996; Meijerink \& Spaans 2005; Aalto et al. 2007; Aalto 2013). It is therefore reasonable to expect that the line ratio $I_{\mathrm{CN}} / I_{\mathrm{HCN}}$ increases from a dense warm gas-dominated ISM to a PDR-dominated ISM and to an XDR-dominated ISM. We arrange the ten galaxies in ascending order by $I_{\mathrm{CN}} / I_{\mathrm{HCN}}$ (see Table 3 ). It is interesting to find that $I_{\mathrm{CN}} / I_{\mathrm{HNC}}, I_{\mathrm{HCN}} / I_{\mathrm{HNC}}$, and $I_{\mathrm{HCO}^{+}} / I_{\mathrm{HNC}}$ increase from $; 7771$ to NGC 6240, then decrease from UGC 2866 to UGC 5101, except for NGC 1614 and NGC 4194. If we tentatively assume that the ISM of NGC 7771 is dominated by warm dense gas, NGC 3079, NGC 3556, and NGC 6240 are dominated by PDRs, and UGC 2866, NGC 660, NGC 7469, and UGC 5101 are dominated by XDRs, and their line ratios and the corresponding variations can be well explained.

For NGC 7771, $I_{\mathrm{HCO}^{+}} / I_{\mathrm{HCN}}<1$ suggests that the ISM is dominated by dense warm gas (Loenen et al. 2006; Baan et al. 2008). $I_{\mathrm{HCN}} / I_{\mathrm{HNC}}$ is greater than the corresponding average value of HMPOCs, but lower than that of UCHIICs. This also supports that the ISM of NGC 7771 is dominated by dense warm gas, where the abundance of HNC decreases with increasing gas temperature owing to the temperature dependence 

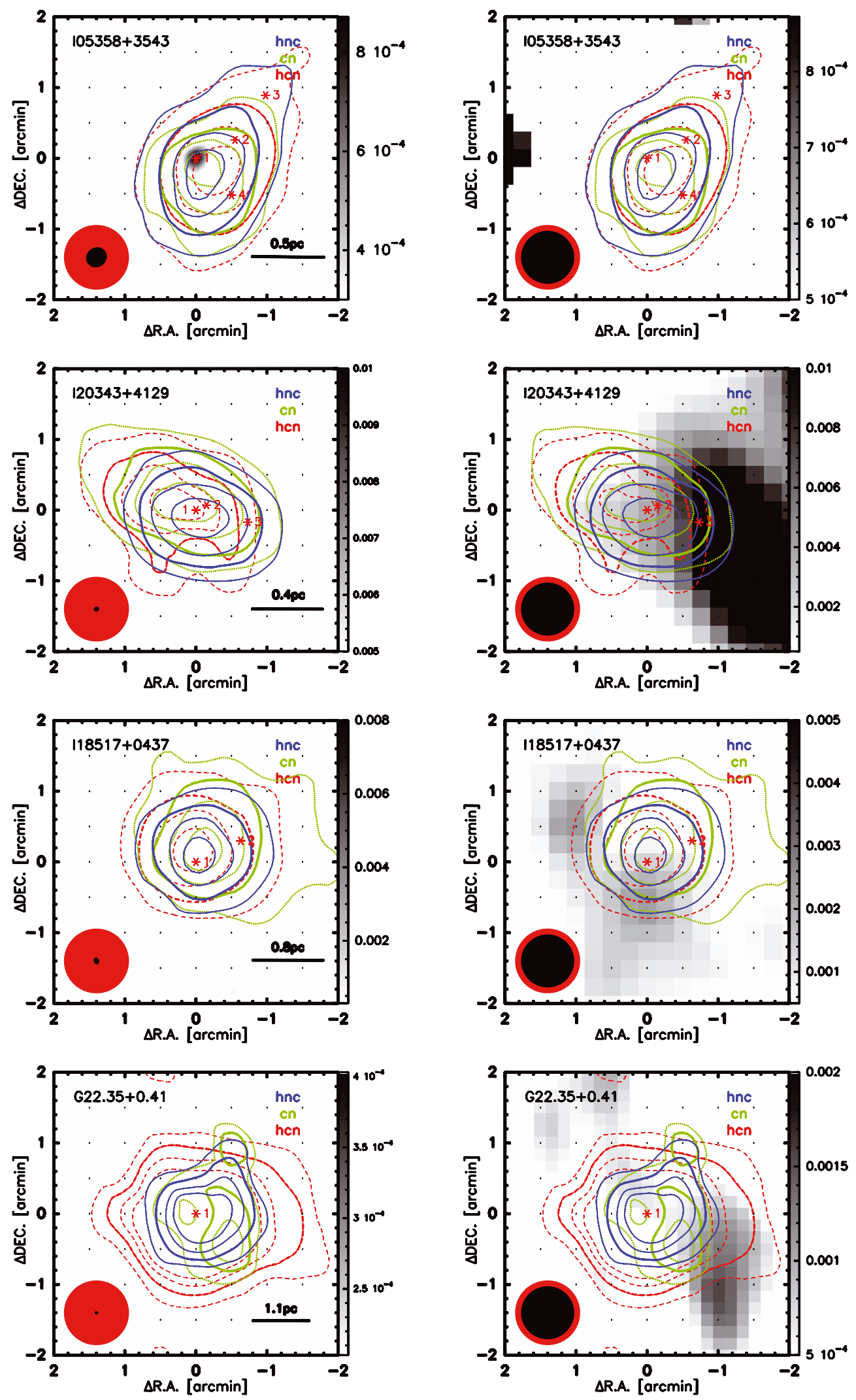

Fig. 6. Contour maps for the integrated intensity of CN 1-0 (green), HCN (red) and HNC 1-0 (blue) for four representative clumps superimposed on their respective continuum map in gray scale at 8.4 (or 4.8) GHz (left) and $1.4 \mathrm{GHz}$ continuum map (right). The contour levels are 30\%, 50\%, $70 \%$, and $90 \%$ of the map peak, the heavy lines represent $50 \%$. Here $\mathrm{CN}(1-0)$, HCN(1-0), and HNC(1-0) emission of G22.35+0.41 is very weak, the corresponding contour levels are $2 \sigma, 3 \sigma$ (heavy line), $4 \sigma$, and $5 \sigma$. The red asterisk in the map indicates the position of HMSC, HMPO, UCHII, and HII regions. The red circle in the bottom left corner represents the FWHM beam size for the molecular lines, the enclosed black circle indicates continuum emission observations. The horizontal lines in the bottom right corner of the left panels indicate the linear scale corresponding to one arcminute at the distance of the respective clump. The unit of the gray scale bar on the right is in Jy/beam. 
of neutral-neutral reactions, and so it is natural to expect $I_{\mathrm{HCN}} / I_{\mathrm{HNC}}>1$ (Costagliola et al. 2011).

For the ISM that is dominated by PDRs, $I_{\mathrm{HCO}^{+}} / I_{\mathrm{HCN}}$ increases from 0.25 to 2 (Baan et al. 2008). Shocks excited by supernova explosions will increase $I_{\mathrm{HCO}^{+}} / I_{\mathrm{HCN}}$ (Loenen et al. 2006; Baan et al. 2008), and mechanical heating by supernovae explosions could also increase $I_{\mathrm{HCN}} / I_{\mathrm{HNC}}$ in PDRs (Loenen et al. 2006). The variations of $I_{\mathrm{HCO}^{+}} / I_{\mathrm{HCN}}$ and $I_{\mathrm{HCN}} / I_{\mathrm{HNC}}$ for NGC 3079, NGC 3556, and NGC 6240 suggest that they are probably dominated by PDRs. $I_{\mathrm{CN}} / I_{\mathrm{HCN}}$ is slightly greater than that of UCHIICs, but $I_{\mathrm{CN}} / I_{\mathrm{HNC}}$ and $I_{\mathrm{HCN}} / I_{\mathrm{HNC}}$ are much greater than that of UCHIICs. One possible reason may be that the star formation efficiency for UCHIIC is much lower than that of ISM dominated by PDRs in external galaxies.

However, when the ISM is dominated by XDRs and so $\mathrm{HCO}^{+}(1-0)$ is destroyed where $\mathrm{HCN}(1-0)$ may show high abundances that cause the $I_{\mathrm{HCO}^{+}} / I_{\mathrm{HCN}}$ to decrease (Lepp \& Dalgarno 1996; Maloney et al. 1996; Aalto et al. 2007). Chemistry dominated by hard X-rays may lead to an enhancement of the HNC abundance (Meijerink et al. 2006). Hence $I_{\mathrm{CN}(1-0)} / I_{\mathrm{HCN}(1-0)}, I_{\mathrm{HCO}^{+}(1-0)} / I_{\mathrm{HCN}(1-0)}$, and $I_{\mathrm{HCN}(1-0)} / I_{\mathrm{HNC}(1-0)}$ values of UGC 2866, NGC 660, NGC 7469, and UGC 5101 suggest that they may be dominated by XDRs. The decrease in $I_{\mathrm{HCO}^{+}(1-0)} / I_{\mathrm{HCN}(1-0)}$ and $I_{\mathrm{HCN}(1-0)} / I_{\mathrm{HNC}(1-0)}$ may be ascribed to increasing X-ray radiation and a decreasing optical depth of the lines. The $\mathrm{CN}(1-0), \mathrm{HCN}(1-0), \mathrm{HNC}(1-0)$, and $\mathrm{HNC}(1-0)$ emissons of NGC 1614 and NGC 4194 are much weaker than those of UGC 2866, NGC 660, NGC 7469, and UGC 5101. They seem to represent a later evolutionary stage of the ISM dominated by XDR, where much molecular gas has been depleted.

This analysis suggests that the global line ratios between $\mathrm{CN}(1-0), \mathrm{HCN}(1-0), \mathrm{HNC}(1-0)$, and $\mathrm{HCO}^{+}(1-0)$ may be used to distinguish the ISM dominated by dense warm gas, PDRs and XDRs. This provides an important tool for studying the evolution of galaxies.

\section{Conclusions}

We have performed $\mathrm{CN}(1-0)$, $\mathrm{HCN}(1-0)$, and $\mathrm{HNC}(1-0)$ mapping observations toward 38 high-mass star-forming regions of different evolutionary stages and identified 5 HMSCCs, 14 HMPOCs, 15 UCHIICs, and 10 HIICs. We studied the global line ratios for these molecular clumps and determined whether the global line ratios $I_{\mathrm{CN}} / I_{\mathrm{HCN}}, I_{\mathrm{CN}} / I_{\mathrm{HNC}}$, and $I_{\mathrm{HCN}} / I_{\mathrm{HNC}}$ are suitable as tracers for the evolution of high-mass starforming regions in the Milky Way. We obtained the following results:

(1) $I_{\mathrm{CN}} / I_{\mathrm{HNC}}$ and $I_{\mathrm{HCN}} / I_{\mathrm{HNC}}$ values for HMSCCs and HMPOCs are sensitive to the $20 \mathrm{~cm}$ continuum emission associated with them, which means the appearance of $\mathrm{O} / \mathrm{B}$ stars. However, $I_{\mathrm{CN}} / I_{\mathrm{HNC}}$ and $I_{\mathrm{HCN}} / I_{\mathrm{HNC}}$ for UCHIICs are not sensitive to the $20 \mathrm{~cm}$ continuum emission associated with them. This suggests that the chemical properties of high-mass starforming regions at early stages are more easily affected by nearby $\mathrm{O} / \mathrm{B}$ stars.

(2) When we removed the HMSCCs and HMPOCs associated with $20 \mathrm{~cm}$ continuum emission, both $I_{\mathrm{CN}} / I_{\mathrm{HNC}}$ and $I_{\mathrm{HCN}} / I_{\mathrm{HNC}}$ increased with the age of star-forming regions. The $I_{\mathrm{CN}} / I_{\mathrm{HNC}}$ values for HMSCCs, HMPOCs, UCHIICs, and HIICs are $0.3 \pm 0.16,0.48 \pm 0.18,1.20 \pm 0.46$, and $1.58 \pm 0.52$, respectively. The corresponding values of
$I_{\mathrm{HCN}} / I_{\mathrm{HNC}}$ are $0.71 \pm 0.32,1.36 \pm 0.35,2.55 \pm 0.79$, and $2.93 \pm 1.07$

(3) We tentatively divided the star-forming regions into two types. Type 1 sources include HMSCCs and HMPOCs that are not associated with external $20 \mathrm{~cm}$ continuum emission. Type 2 sources include all UCHIICs and HIICs. The line ratios $I_{\mathrm{CN}} / I_{\mathrm{HNC}}$ and $I_{\mathrm{HCN}} / I_{\mathrm{HNC}}$ can trace the evolution from type 1 to type 2 well.

(4) The line ratio $I_{\mathrm{CN}} / I_{\mathrm{HCN}}$ is not sensitive to the evolution of high-mass star-forming regions in the Milky Way.

(5) The global line ratios between $\mathrm{CN}(1-0), \operatorname{HCN}(1-0)$, $\mathrm{HNC}(1-0)$, and $\mathrm{HCO}^{+}(1-0)$ may be used to distinguish the ISM of the galaxies dominated by dense warm gas, PDRs, and XDRs.

Acknowledgements. We thank Ye Xu and Xing-Wu Zheng for their help. We are very grateful to the staff of Qing hai Station at Purple Mountain Observatory for their assistance with the observations and data reductions. We thank Xiangjun Shao, Juan Li, and Zhi-yu Zhang for help with VLA data reduction, and Rai Yuen for reading the manuscript. This work was funded by The National Natural Science foundation of China under Grant 11433008 and partly supported by National Basic Research Program of China (973 program, 2012CB821802) and the National Natural Science foundation of China under Grant 11373062 and 11303081, and The Program of The Light in China's Western Region under grant Nos. RCPY201202 and XBBS-2014-24.

\section{References}

Aalto, S. 2013, IAUS, 292, 199

Aalto, S., Polatidis, A. G., Hüttemeister S., \& Curran, S. J. 2002, A\&A, 381, 783

Aalto, S., Monje R., \& Martin, S. 2007, A\&A, 475, 479

Anderson, L. D., \& Bania, T. M. 2009, ApJ, 690, 706

Araya, E., Hofner, P., Churchwell, E., \& Kurtz, S. 2002, ApJS, 138, 63

Baan, W. A., Henkel, C., Loenen, A. F., Baudry, A., \& Wiklind, T. 2008, A\&A, 477,747

Baan, W. A., Loenen, A. F., \& Spaans, M. 2010, A\&A, 516, A40

Beuther, H., Schilke, P., Menten, K. M., et al. 2002, ApJ, 566, 945

Boger G. I. \& Sternberg, A. 2005, ApJ, 632, 302

Bronfman, L., Nyman, L.-A., \& May, J. 1996, A\&AS, 115, 81

Caselli, P. 2011, IAUS, 280, 19

Condon, J. J., Cotton, W. D., Greisen, E. W., et al. 1998, AJ, 115, 1693

Costagliola, F., Aalto, S., Rodriguez, M. I., et al. 2011, A\&A, 528, A30

Fich, M., \& Blitz, L. 1984, ApJ, 279, 125

Fuente, A., Martìn-Pintado, J., Cernicharo, J., \& Bachiller, R. 1993, A\&A, 276, 473

Gerner, T., Beuther, H., Semenov, D., et al. 2014, A\&A, 563, A97

Goldsmith, P. F., Hjalmarson A., \& Ellder, J. 1986, ApJ, 310, 685

Helfand, D. J., Becker, R. H., White, R. L., Fallon, A., \& Tuttle, S. 2006, AJ, 131,2525

Helmich, F. P., \& van Dishoeck, E. F. 1997, A\&AS, 124, 205

Heyer, M. H., Carpenter, J. M., \& Snell, R. L. 2001, ApJ, 551, 852

Hoq, S., Jackson, J. M., Foster, J. B., et al. 2013, ApJ, 777, 157

Hou, L. G., Han, J. L., \& Shi, W. B. 2009, A\&A, 499, 473

Jansen, D. J., van Dishoeck, E. F., Black, J. H., Spaans, M., \& Sosin, C. 1995, A\&A, 302, 223

Kurtz, S. E., Watson, A. M., Hofner, P., \& Otte, B. 1999, ApJ, 514, 232

Lepp, S., \& Dalgarno, A. 1996, A\&A, 306, L21

Loenen, A. F., Baan, W. A., \& Spaans, M. 2006, A\&A, 458, 89

Maloney, P. R., Hollenbach, D. J., \& Tielens, A. G. G. M. 1996, ApJ, 466, 561

Meijerink, R., \& Spaans, M. 2005, A\&A, 436, 397

Meijerink, R., Spaans, M., \& Israel, F. P. 2006, ApJ, 650, L103

Rathborne J. M., Jackson J. M., \& Simon, R. 2006, ApJ, 641, 389

Reiter, M. R., Shirley, Y. L., Wu, J., et al. 2011, ApJS, 195, 1

Rodriguez-Franco, A., Martin-Pintado, J., \& Fuente, A. 1998, A\&A, 329, 1097

Russeil, D., Adami, C., \& Georgelin, Y. M. 2007, A\&A, 470, 161

Sakai, T., Sakai, N., Kamegai, K., et al. 2008, ApJ, 678, 1049

Sanhueza, P., Jackson, J. M., Foster, J. B., et al. 2012, ApJ, 756, 60

Schilke, P., Walmsley, C. M., Pineau des Forets, G., et al. 1992, A\&A, 256, 595 
Shan, W. L., Yang, J., Shi, S. C., et al. 2010, in Twenty-First International Symp. on Space Terahertz Technology, 136

Sridharan, T. K., Beuther, H., Schilke, P., Menten, K. M., \& Wyrowski, F. 2002, ApJ, 566, 931

Sridharan, T. K., Beuther, H., Saito, M., Wyrowski, F., \& Schilke, P. 2005, ApJ, 634, L57

Sternberg, A., \& Dalgarno, A. 1995, ApJS, 99, 565

Tan, J. C., Beltran, M. T., Caselli, P., et al. 2014, in Protostars and Planets VI, eds. H. Beuther, R. S. Klessen, C. P. Dullemond, \& T. Henning (University of Arizona Press), in press [arXiv: 1402 .0919]
Thi, W.-F., van Zadelhoff, G.-J., \& van Dishoeck, E. F. 2004, A\&A, 425, 955 Ungerechts, H., Bergin, E. A., Goldsmith, P. F., et al. 1997, ApJ, 482, 245

van Zadelhoff, G.-J., Aikawa, Y., Hogerheijde, M. R., \& van Dishoeck, E. F 2003, A\&A, 397, 789

Vasyunina, T., Linz, H., Henning, Th., et al. 2011, ApJ, 527, 88

White, R. L., Becker, R. H., \& Helfand, D. J. 2005, AJ, 130, 586

Wood, D. O. S., \& Churchwell, E. 1989, ApJS, 69, 831

Xu, Y., Voronkov, M. A., Pandian, J. D., et al. 2009, A\&A, 507, 1117

Xue, R., \& Wu, Y. F. 2008, ApJ, 680, 446

Zinnecker, H., \& Yorke, H. W. 2007, ARA\&A, 45, 481 
X. H. Han et al.: A possible indicator for high-mass star formation sequence

\section{Appendix A: Tables}

Table A.1. Information for observed sources in $\mathrm{cm}$ and $\mathrm{mm}$ wavelengths and their evolutionary stages.

\begin{tabular}{|c|c|c|c|c|c|c|c|c|c|c|c|c|c|c|c|c|c|}
\hline \multirow{2}{*}{$\begin{array}{l}\text { Source } \\
\text { G22.35+0.41-1 }\end{array}$} & \multicolumn{3}{|c|}{ RA (J2000) } & \multicolumn{3}{|c|}{$\operatorname{Dec}(\mathrm{J} 2000)$} & \multirow[t]{2}{*}{ Radio source } & \multicolumn{3}{|c|}{$\mathrm{RA}(\mathrm{J} 2000)$} & & $\mathrm{ec}(\mathrm{J} 2$ & 2000) & $8 \mu \mathrm{m}$ & $\mathrm{cm}$ & Offset $(\mathrm{cm} / \mathrm{mm})$ & Evolutionary stage \\
\hline & 18 & 30 & 24.4 & -09 & 10 & $34^{a}$ & & & & & & & & $\mathrm{D}^{a}$ & $\mathrm{~N}$ & & $\mathrm{HMSC}^{a}$ \\
\hline $\mathrm{G} 23.60+0.00-1$ & 18 & 34 & 11.6 & -08 & 19 & $06^{a}$ & & & & & & & & $\mathrm{D}^{a}$ & $\mathrm{~N}$ & & $\mathrm{HMSC}^{a}$ \\
\hline $\mathrm{G} 23.60+0.00-2$ & 18 & 34 & 21.1 & -08 & 18 & $07^{a}$ & & & & & & & & $\mathrm{D}^{a}$ & $\mathrm{~N}$ & & $\mathrm{HMSC}^{a}$ \\
\hline $\mathrm{G} 23.60+0.00-3$ & 18 & 34 & 10.0 & -08 & 18 & $28^{a}$ & & & & & & & & $-^{a}$ & $\mathrm{~N}$ & & $\operatorname{HMSC}^{a}$ \\
\hline $\mathrm{G} 23.60+0.00-4$ & 18 & 34 & 23.0 & -08 & 18 & $21^{a}$ & & & & & & & & $-^{a}$ & $\mathrm{~N}$ & & $\mathrm{HMSC}^{a}$ \\
\hline $\mathrm{G} 23.60+0.00-5$ & 18 & 34 & 09.5 & -08 & 18 & $00^{a}$ & & 18 & 34 & 09.8 & -08 & 17 & 52.11 & $\mathrm{~S}$ & $\mathrm{~S}$ & 9.08 & UCHII \\
\hline $\mathrm{G} 23.60+0.00-7$ & 18 & 34 & 21.1 & -08 & 17 & $11^{a}$ & & & & & & & & $\mathrm{D}^{a}$ & $\mathrm{~N}$ & & $\mathrm{HMSC}^{a}$ \\
\hline $\mathrm{G} 34.43+0.24-1$ & 18 & 53 & 18.0 & 01 & 25 & $24^{a}$ & & & & & & & & $-^{a}$ & $\mathrm{~N}$ & & $\mathrm{HMSC}^{a}$ \\
\hline $\mathrm{G} 34.43+0.24-2$ & 18 & 53 & 18.6 & 01 & 24 & $40^{a}$ & $\mathrm{G} 34.402+0.233$ & 18 & 53 & 18.7 & 01 & 24 & 47.6 & $\mathrm{~S}^{a}$ & $\mathrm{~W}$ & 7.75 & HMPO \\
\hline $\mathrm{G} 34.43+0.24-3$ & 18 & 53 & 20.4 & 01 & 28 & $23^{a}$ & & & & & & & & $\mathrm{D}^{a}$ & $\mathrm{~N}$ & & $\mathrm{HMSC}^{a}$ \\
\hline $\mathrm{G} 34.43+0.24-4$ & 18 & 53 & 19.0 & 01 & 24 & $08^{a}$ & & & & & & & & $\AA^{a}$ & $\mathrm{~N}$ & & $\mathrm{HMSC}^{a}$ \\
\hline $\mathrm{G} 34.43+0.24-5$ & 18 & 53 & 19.8 & 01 & 23 & $30^{a}$ & & & & & & & & $-^{a}$ & $\mathrm{~N}$ & & $\mathrm{HMSC}^{a}$ \\
\hline $\mathrm{G} 34.43+0.24-6$ & 18 & 53 & 18.6 & 01 & 27 & $48^{a}$ & & & & & & & & $\mathrm{D}^{a}$ & $\mathrm{~N}$ & & $\mathrm{HMSC}^{a}$ \\
\hline $\mathrm{G} 34.43+0.24-7$ & 18 & 53 & 18.3 & 01 & 27 & $13^{a}$ & & & & & & & & - & $\mathrm{N}$ & & $\mathrm{HMSC}^{a}$ \\
\hline $\mathrm{G} 34.43+0.24-8$ & 18 & 53 & 16.4 & 01 & 26 & $20^{a}$ & & & & & & & & $\mathrm{D}^{a}$ & $\mathrm{~N}$ & & $\mathrm{HMSC}^{a}$ \\
\hline $\mathrm{G} 34.43+0.24-9$ & 18 & 53 & 18.4 & 01 & 28 & $14^{a}$ & & & & & & & & $\mathrm{D}^{a}$ & $\mathrm{~N}$ & & $\mathrm{HMSC}^{a}$ \\
\hline I18151-1208 - 1 & 18 & 17 & 58.0 & -12 & 07 & $27^{b}$ & G18.340 + 1.772 & & & & & & & $\mathrm{~S}^{a}$ & $\mathrm{~W}^{e}$ & & $\mathrm{HMPO}^{e}$ \\
\hline I18151-1208 - 2 & 18 & 17 & 50.4 & -12 & 07 & $55^{b}$ & & & & & & & & $\mathrm{D}^{a}$ & $\mathrm{~N}$ & & $\mathrm{HMSC}^{c}$ \\
\hline I18151-1208-3 & 18 & 17 & 52.2 & -12 & 06 & $56^{b}$ & & & & & & & & $\mathrm{~S}^{a}$ & $\mathrm{~N}$ & & HMPO \\
\hline I1 $8223-1243-1$ & 18 & 25 & 10.5 & -12 & 42 & $26^{b}$ & G18.657 - 0.060 & 18 & 25 & 10.4 & -12 & 42 & 15.4 & $\mathrm{~S}^{a}$ & $\mathrm{~S}^{e}$ & 10.6 & $\mathrm{HMPO} / \mathrm{UCHII}^{e}$ \\
\hline $\mathrm{I} 18223-1243-2$ & 18 & 25 & 09.5 & -12 & 44 & $15^{b}$ & & & & & & & & $\mathrm{D}^{a}$ & $\mathrm{~N}$ & & $\mathrm{HMSC}^{c}$ \\
\hline I1 $8223-1243-3$ & 18 & 25 & 08.3 & -12 & 45 & $28^{b}$ & & & & & & & & $\mathrm{D}^{a}$ & $\mathrm{~N}$ & & $\mathrm{HMSC}^{c}$ \\
\hline I1 $8223-1243-4$ & 18 & 25 & 07.2 & -12 & 47 & $54^{b}$ & & & & & & & & $\mathrm{D}^{a}$ & $\mathrm{~N}$ & & $\mathrm{HMSC}^{c}$ \\
\hline I18306 - $0835-1$ & 18 & 33 & 24.0 & -08 & 33 & $31^{b}$ & $\mathrm{G} 23.260+0.084$ & 18 & 33 & 23.94 & -08 & 33 & 33.10 & $\mathrm{~S}^{a}$ & $\mathrm{~S}^{e}$ & 2.28 & $\mathrm{HMPO} / \mathrm{UCHII}{ }^{e}$ \\
\hline I18310 - 0825- 1 & 18 & 33 & 47.2 & -08 & 23 & $35^{b}$ & $\mathrm{G} 23.457+0.068$ & 18 & 33 & 47.83 & -08 & 23 & 35.17 & $\mathrm{~S}$ & $\mathrm{~W}^{e}$ & 9.45 & $\mathrm{HMPO}^{e}$ \\
\hline I18310 - $0825-2$ & 18 & 33 & 44.1 & -08 & 21 & $19.6^{b}$ & & & & & & & & $\mathrm{~S}$ & $\mathrm{~N}$ & & HMPO \\
\hline I18310 - 0825- 3 & 18 & 33 & 42.2 & -08 & 21 & $35.7^{b}$ & & & & & & & & $\mathrm{D}$ & $\mathrm{N}$ & & HMSC \\
\hline I1 $8310-0825-4$ & 18 & 33 & 39.5 & -08 & 21 & $10.4^{b}$ & & & & & & & & $\mathrm{D}^{b}$ & $\mathrm{~N}$ & & $\mathrm{HMSC}^{c}$ \\
\hline I18337 - $0743-1$ & 18 & 36 & 41.0 & -07 & 39 & $20^{b}$ & G24.401 - 0.194 & 18 & 36 & 46.77 & -07 & 35 & $41.16^{*}$ & $\mathrm{~S}^{a}$ & $\mathrm{~N}$ & & $\mathrm{HMPO}^{e}$ \\
\hline I18337 - $0743-5$ & 18 & 36 & 41.0 & -07 & 39 & $56^{c}$ & & & & & & & & $\mathrm{D}^{b}$ & $\mathrm{~N}$ & & $\mathrm{HMSC}^{c}$ \\
\hline $\mathrm{I} 18530+0215-1$ & 18 & 55 & 34.0 & 02 & 19 & $10.1^{c}$ & $\mathrm{G} 35.467+0.139$ & 18 & 55 & 34.1 & 02 & 19 & 10.3 & S & $\mathrm{S}^{e}$ & 1.51 & $\mathrm{HMPO} / \mathrm{UCHII}^{e}$ \\
\hline $\mathrm{I} 18530+0215-2$ & 18 & 55 & 29.0 & 02 & 17 & $43^{b}$ & & & & & & & & $\mathrm{D}^{b}$ & $\mathrm{~N}$ & & $\mathrm{HMSC}^{e}$ \\
\hline I05358 + 3543-1 & 05 & 39 & 13.0 & 35 & 45 & $49.4^{b}$ & $\mathrm{G} 173.484+2.434$ & 05 & 39 & 13.0 & 35 & 45 & 49.4 & $\mathrm{~S}$ & $\mathrm{~W}^{e}$ & & $\mathrm{HMPO}^{e}$ \\
\hline I05358 + 3543-2 & 05 & 39 & 10.8 & 35 & 46 & $04.7^{b}$ & & & & & & & & $\mathrm{D}$ & $\mathrm{N}$ & & HMSC \\
\hline I05358 + 3543-3 & 05 & 39 & 09.1 & 35 & 46 & $42.9^{b}$ & & & & & & & & $\mathrm{D}$ & $\mathrm{N}$ & & HMSC \\
\hline I05358 + 3543-4 & 05 & 39 & 11.0 & 35 & 45 & $18.2^{b}$ & & & & & & & & $\mathrm{~S}$ & $\mathrm{~N}$ & & HMPO \\
\hline I18445 - 0222-1 & 18 & 47 & 10.8 & -02 & 19 & $06^{b}$ & G30.383 - 0.110 & 18 & 47 & 10.57 & -02 & 19 & 7.69 & $\mathrm{~S}$ & $\mathrm{~S}^{e}$ & 3.84 & $\mathrm{HMPO} / \mathrm{UCHII}^{e}$ \\
\hline $\mathrm{I} 18517+0437-1$ & 18 & 54 & 14.3 & 04 & 41 & $39.7^{b}$ & $\mathrm{G} 37.427+1.518$ & 18 & 54 & 14.50 & 04 & 41 & 22.74 & $\mathrm{~S}$ & $\mathrm{~W}^{e}$ & 5.10 & $\mathrm{HMPO}^{e}$ \\
\hline $\mathrm{I} 18517+0437-2$ & 18 & 54 & 11.8 & 04 & 41 & $57.7^{b}$ & & & & & & & & - & $\mathrm{N}$ & & $\mathrm{HMSC}^{c}$ \\
\hline $\mathrm{I} 20126+4104$ & 20 & 14 & 26.0 & 41 & 13 & $32.5^{b}$ & G78.122 + 3.633 & 20 & 14 & 26.0 & 41 & 13 & 32.46 & $\mathrm{~S}$ & $\mathrm{~W}^{f}$ & & $\mathrm{HMPO}^{e}$ \\
\hline $\mathrm{I} 20293+3952-1$ & 20 & 31 & 10.7 & 40 & 03 & $10.0^{b}$ & $\mathrm{G} 78.975+0.356$ & 20 & 31 & 11.2 & 40 & 03 & 9.9 & $\mathrm{~S}$ & $\mathrm{~W}^{f}$ & 7.5 & $\mathrm{HMPO}^{e}$ \\
\hline $\mathrm{I} 20293+3952-2$ & 20 & 31 & 09.8 & 40 & 03 & $41.2^{b}$ & & & & & & & & $\mathrm{~S}$ & $\mathrm{~N}$ & & HMPO \\
\hline $\mathrm{I} 20343+4129-1$ & 20 & 36 & 07.7 & 41 & 39 & $56.7^{b}$ & & & & & & & & $\mathrm{~S}$ & $\mathrm{~W}^{f}$ & & $\mathrm{HMPO}^{e}$ \\
\hline $\mathrm{I} 20343+4129-2$ & 20 & 36 & 07.1 & 41 & 40 & $01.0^{b}$ & G80.828+0.568 & & & & & & & S & $\mathrm{N}$ & & HMPO \\
\hline $\mathrm{I} 20343+4129-3$ & 20 & 36 & 04.8 & 41 & 39 & $46.6^{b}$ & & & & & & & & $\mathrm{~S}$ & $\mathrm{~N}$ & & HMPO \\
\hline $\mathrm{I} 22134+5834$ & 22 & 15 & 09.2 & 58 & 49 & $09.0^{b}$ & $\mathrm{G} 103.876+1.856$ & 22 & 15 & 9.20 & 58 & 49 & 09.0 & $\mathrm{~S}$ & $\mathrm{~N}^{f}$ & & $\mathrm{HMPO}^{e}$ \\
\hline$I 22551+6221$ & 22 & 57 & 01.4 & 62 & 38 & $28.1^{b}$ & $\mathrm{G} 110.210+2.616$ & 22 & 57 & 6.18 & 62 & 37 & 54.4 & $\mathrm{~S}$ & $\mathrm{~S}^{f}$ & 10.27 & $\mathrm{HMPO} / \mathrm{UCHII}^{e}$ \\
\hline $\mathrm{I} 22570+5912-1$ & 22 & 59 & 05.93 & 59 & 28 & $19.2^{b}$ & G109.098 - 0.346 & 22 & 59 & 6.48 & 59 & 28 & 34.3 & $\mathrm{~S}$ & $\mathrm{~S}^{f}$ & 17.21 & $\mathrm{HMPO} / \mathrm{UCHII}^{e}$ \\
\hline $\mathrm{I} 22570+5912-2$ & 22 & 59 & 02.79 & 59 & 27 & $35.6^{b}$ & & & & & & & & $\mathrm{~S}$ & $\mathrm{~N}$ & & HMPO \\
\hline $\mathrm{I} 22570+5912-3$ & 22 & 58 & 59.27 & 59 & 28 & $32.6^{b}$ & & & & & & & & - & $\mathrm{N}$ & & HMSC \\
\hline $\mathrm{I} 22570+5912-4$ & 22 & 58 & 57.85 & 59 & 28 & $49^{b}$ & & & & & & & & $\mathrm{~S}$ & $\mathrm{~N}$ & & HMPO \\
\hline $\mathrm{I} 23033+5951$ & 23 & 05 & 25.0 & 60 & 08 & $15.9^{b}$ & G110.093-0.065 & & & & & & & $\mathrm{S}$ & $\mathrm{W}^{f}$ & & $\mathrm{HMPO}^{e}$ \\
\hline $\mathrm{I} 23139+5939$ & 23 & 16 & 10.4 & 59 & 55 & $28.7^{b}$ & G111.253 - 0.770 & 23 & 16 & 10.4 & 59 & 55 & 28.76 & $\mathrm{~S}$ & $\mathrm{~W}^{e}$ & & $\mathrm{HMPO}^{e}$ \\
\hline $\mathrm{I} 01202+6133$ & 01 & 23 & 32.3 & 61 & 48 & $48.7^{b}$ & G126.713 - 0.822 & & & & & & & $\mathrm{~S}$ & W & & HMPO \\
\hline I03035 + 5819 & & & & & & & $\mathrm{G} 139.912+0.2$ & 03 & 07 & 23.7 & 58 & 30 & 50.1 & $\mathrm{~S}$ & $\mathrm{~S}$ & & UCHII \\
\hline $\mathrm{I} 19078+0901-1$ & & & & & & & $\mathrm{G} 43.169+0.004$ & 19 & 10 & 13.4 & 09 & 06 & 14.3 & $\mathrm{~S}$ & $\mathrm{~S}$ & & UCHII \\
\hline $\mathrm{I} 19078+0901-2$ & & & & & & & & 19 & 10 & 21.8 & 09 & 04 & 59.1 & S & $\mathrm{S}$ & & UCHII \\
\hline $\mathrm{I} 19078+0901-3$ & & & & & & & & 19 & 10 & 10.7 & 09 & 05 & 14.2 & $\mathrm{~S}$ & $\mathrm{~S}$ & & UCHII \\
\hline $\mathrm{I} 19097+0847-1$ & & & & & & & G43.178-0.518 & 19 & 12 & 11.3 & 08 & 52 & 17.7 & $\mathrm{~S}$ & $\mathrm{~S}^{g}$ & & $\mathrm{UCHII}^{g}$ \\
\hline $\mathrm{I} 19097+0847-2$ & & & & & & & G43.186- 0.526 & 19 & 12 & 08.8 & 08 & 52 & 08.3 & $\mathrm{~S}$ & $\mathrm{~S}$ & & UCHII \\
\hline $\mathrm{I} 19110+1045$ & & & & & & & $\mathrm{G} 45.071+0.132$ & 19 & 13 & 20.1 & 10 & 50 & 52.84 & $\mathrm{~S}$ & $\mathrm{~S}^{g}$ & & $\mathrm{UCHII}^{g}$ \\
\hline $\mathrm{I} 19111+1048$ & & & & & & & $\mathrm{G} 45.122+0.132$ & 19 & 13 & 27.9 & 10 & 53 & 34.4 & $\mathrm{~S}$ & $\mathrm{~S}^{g}$ & & $\mathrm{UCHII}^{g}$ \\
\hline $\mathrm{I} 19442+2427-1$ & & & & & & & G60.883 - 0.129 & 19 & 46 & 20.1 & 24 & 35 & 28.9 & $\mathrm{~S}$ & $\mathrm{~S}^{h}$ & & $\mathrm{UCHII}^{h}$ \\
\hline $\mathrm{I} 19442+2427-2$ & 19 & 46 & 22.3 & 24 & 36 & $01^{d}$ & & & & & & & & - & $\mathrm{N}$ & & $\mathrm{HMSC}^{h}$ \\
\hline $\mathrm{I} 19442+2427-3$ & 19 & 46 & 23.2 & 24 & 36 & $28^{d}$ & & & & & & & & $S$ & $\mathrm{~N}$ & & $\mathrm{HMPO}^{h}$ \\
\hline $\mathrm{I} 20081+3122$ & & & & & & & G69.540 - 0.976 & 20 & 10 & 09.1 & 31 & 31 & 35.7 & $\mathrm{~S}$ & $\mathrm{~S}^{h}$ & & $\mathrm{UCHII}^{h}$ \\
\hline $\mathrm{I} 23116+6111$ & & & & & & & $\mathrm{G} 111.543+0.779$ & 23 & 13 & 45.4 & 61 & 28 & 19.7 & $\mathrm{~S}$ & $\mathrm{~S}^{h}$ & & $\mathrm{UCHII}^{h}$ \\
\hline $\mathrm{I} 23133+6050$ & & & & & & & $\mathrm{G} 111.612+0.373$ & 23 & 15 & 31.2 & 61 & 07 & 10.6 & $\mathrm{~S}$ & $\mathrm{~S}^{h}$ & & $\mathrm{UCHII}^{h}$ \\
\hline $\mathrm{I} 23138+5945$ & & & & & & & G111.284-0.664 & 23 & 16 & 04.0 & 60 & 02 & 01.2 & $\mathrm{~S}$ & $\mathrm{~S}^{h}$ & & $\mathrm{UCHII}^{h}$ \\
\hline
\end{tabular}

Notes. From left to right, the columns are (1) sources name; (2) right ascension of $1.2 \mathrm{~mm}$; (3) declination of $1.2 \mathrm{~mm}$; (4) radio source near the detected source; (5) right ascension of $\mathrm{cm}$ wave continuum emission; (6) declination of $\mathrm{cm}$ wave continuum emission; (7) information for $8 \mu \mathrm{m}$; (8) information for $\mathrm{cm}$ wave continuum emission; (9) positional comparison between CM/MM sources; (10) evolutionary stage of single core. The D, S, and "-" in Col. (7) indicate dark object, point-like sources and objects that are neither point-like source nor dark spot. The W, S, and N in Col. (8) signify that the $\mathrm{cm}$ wave continuum emission is weak, strong, and no emission.

References. (a) Rathborne et al. (2006); (b) Beuther et al. (2002); (c) Sridharan et al. (2005); (d) Xue \& Wu (2008); (e) Sridharan et al. (2002); (f) Sakai et al. (2008); (g) Wood \& Churchwell (1989); (h) Kurtz et al. (1999). 
A\&A 576, A131 (2015)

Table A.2. Observing information for the VLA archival data and the results for radio continuum emission.

\begin{tabular}{|c|c|c|c|c|c|c|c|c|c|c|c|}
\hline Source & Date & Configuration & $\begin{array}{l}\text { Freq } \\
(\mathrm{GHz})\end{array}$ & Project & $\begin{array}{c}\text { Synthesized Beam } \\
\left({ }^{\prime} \times{ }^{\prime \prime},, \circ\right)\end{array}$ & $\begin{array}{c}\mathrm{rms} \\
\left(\mathrm{mJy} \mathrm{B}^{-1}\right)\end{array}$ & $\begin{array}{c}S_{p} \\
\left(\mathrm{mJy} \mathrm{B}^{-1}\right)\end{array}$ & $\begin{array}{l}S_{v} \\
(\mathrm{mJy})\end{array}$ & $\begin{array}{c}\text { Size } \\
\left({ }^{\prime \prime} \times{ }^{\prime \prime}\right)\end{array}$ & $\begin{array}{c}\text { Distance } \\
(\mathrm{kpc})\end{array}$ & $\begin{array}{c}\text { Linear size } \\
(\mathrm{pc})\end{array}$ \\
\hline $\mathrm{G} 22.35+0.41$ & 2008 Mar. 24 & VLA:C:1 & 8.4 & AJ345 & $3.0 \times 2.5,3.7$ & 0.04 & & & & $3.7^{c}$ & \\
\hline $\mathrm{G} 23.60+0.00 \mathrm{~A}$ & 2008 May 09 & VLA:C:1 & 8.4 & AJ345 & $3.9 \times 3.0,2.5$ & 0.18 & & & & $3.7^{c}$ & \\
\hline $\mathrm{G} 23.60+0.00 \mathrm{~B}$ & 2008 May 09 & VLA:C:1 & 8.4 & AJ345 & $3.9 \times 3.0,2.5$ & 0.18 & 1.2 & 16.9 & $13.0 \times 12.0$ & $6.6^{c}$ & 0.42 \\
\hline $\mathrm{G} 34.43+0.24 \mathrm{~A}$ & 2005 Apr. 10 & VLA:B:1 & 8.4 & AG678 & $1.3 \times 1.2,-81$ & 0.02 & & & & $3.6^{c}$ & \\
\hline $\mathrm{G} 34.43+0.24 \mathrm{~B}$ & 2005 Apr. 10 & VLA:B:1 & 8.4 & AG678 & $1.3 \times 1.2,-81$ & 0.02 & 0.09 & 0.2 & $1.1 \times 1.0$ & $3.6^{c}$ & 0.019 \\
\hline I18151-1208A & 1998 Jul 02 & VLA:AB:1 & 4.8 & AS643 & $5.9 \times 2.2,42$ & 0.04 & 0.1 & 0.8 & $4.6 \times 0.9$ & $2.8^{b}$ & 0.062 \\
\hline I18151-1208B & 1998 Jul 02 & VLA:AB:1 & 4.8 & AS643 & $5.9 \times 2.2,42$ & 0.04 & & & & $2.6^{b}$ & \\
\hline I18223-1243A & 1987 Feb 17 & VLA:CD:1 & 8.4 & AC177 & $16.3 \times 9.1,-62$ & 0.22 & & & & $12.4^{b . d}$ & \\
\hline I18223-1243B & 1987 Feb 17 & VLA:CD:1 & 8.4 & AC177 & $16.3 \times 9.1,-62$ & 0.22 & 24.0 & 126.8 & $28.6 \times 25.5$ & $12.4^{b . d}$ & 1.72 \\
\hline I18306-0835 & 1993 Mar. 14 & VLA:B:1 & 8.4 & AF244 & $1.2 \times 0.9,-17$ & 0.10 & 6.8 & 65.6 & $3.3 \times 1.9$ & $10.7^{b . d}$ & 0.17 \\
\hline I18310-0825A & 2005 Jan. 17 & VLA:AB:1 & 8.4 & AC761 & $1.0 \times 0.4,69$ & 0.03 & & & & $5.2^{a . *}$ & \\
\hline I18310-0825B & 2005 Jan. 17 & VLA:AB:1 & 8.4 & AC761 & $1.0 \times 0.4,69$ & 0.03 & 1.4 & 4.2 & $1.0 \times 0.6$ & $5.2^{a . *}$ & 0.025 \\
\hline I18337-0743 & 1990 Dec 08 & VLA:C:1 & 4.8 & AB573 & $6.9 \times 5.1,-2.9$ & 0.38 & & $<1$ & & $11.5^{b . d}$ & \\
\hline $\mathrm{I} 18530+0215$ & 1990 Dec 08 & VLA:C:1 & 4.8 & AB573 & $8.0 \times 5.1,39$ & 0.34 & 124.4 & 198.4 & $4.9 \times 4.5$ & $5.1^{b . d}$ & 0.12 \\
\hline I05358+3543 & 1988 Aug. 02 & VLA:D:1 : 27 & 4.8 & AS341 & $18.0 \times 16.5,-35$ & 0.06 & 0.9 & 1.1 & $11.2 \times 5.2$ & $1.8^{b}$ & 0.097 \\
\hline I18445-0222 & 1990 Dec 08 & VLA:C:1 & 4.8 & AB573 & $6.8 \times 4.9,19$ & 0.11 & 4.8 & 39.7 & $17.6 \times 13.4$ & $5.3^{b . d}$ & 0.45 \\
\hline I18517+0437 & 1994 Oct 04 & VLA:BC:1 & 4.8 & AM462 & $5.8 \times 4.6,71$ & 0.01 & & $<1$ & & $2.9^{b}$ & \\
\hline I20126+4104 & 2005 Aug. 20 & VLA:C:1 & 8.4 & AS831 & $3.0 \times 2.7,-57$ & 0.03 & 0.3 & $<1$ & & $1.7^{b}$ & \\
\hline I $20293+3952$ & 2005 Aug. 20 & VLA:C:1 & 8.4 & AS831 & $2.4 \times 2.3,-80$ & 0.02 & 1.3 & 3.9 & $3.5 \times 3.1$ & $1.3^{b *}$ & 0.022 \\
\hline I $20343+4129$ & 1989 Jul 24 & VLA:BC:1 : 27 & 4.8 & AS374 & $4.6 \times 4.1,-8.3$ & 0.05 & 0.5 & 0.7 & $0.7 \times 0.0$ & $1.4^{b}$ & 0.005 \\
\hline I22134+5834 & 1992 Jan. 06 & VLA:B:1 : 27 & 8.4 & AC295 & $1.1 \times 0.9,-84$ & 0.13 & 1.4 & 1.7 & $0.4 \times 0.2$ & $2.6^{b}$ & 0.005 \\
\hline $\mathrm{I} 22551+6221$ & 1993 Nov 02 & VLA:D: $1: 26$ & 8.4 & AF251 & $5.4 \times 5.4,45$ & 0.08 & 8.2 & 46.1 & $17.4 \times 16.2$ & $0.7^{b}$ & 0.059 \\
\hline I $22570+5912$ & 1988 Jul 20 & VLA:D:1 & 4.8 & AF156 & $13.8 \times 11.0,-11$ & 0.10 & 11.1 & 18.3 & $13.2 \times 5.9$ & $5.1^{b}$ & 0.33 \\
\hline $\mathrm{I} 23033+5951$ & 1998 Jul 02 & VLA:AB: 1 & 4.8 & AS643 & $1.3 \times 0.9,-73$ & 0.03 & 0.4 & 0.6 & $1.4 \times 0.0$ & $3.5^{b}$ & 0.024 \\
\hline I23139+5939 & 1991 Mar. 31 & VLA:D:1 & 8.4 & AB601 & $11.1 \times 8.5,-1.6$ & 0.04 & 0.9 & 1.2 & $4.8 \times 4.2$ & $4.8^{b}$ & 0.11 \\
\hline $\mathrm{I} 01202+6133$ & 2008 Aug. 05 & VLA:D:1 & 8.4 & AL723 & $10.6 \times 10.0,42$ & 0.08 & 0.4 & 0.3 & $2.6 \times 0.3$ & $1.44^{i}$ & 0.018 \\
\hline I03035+5819 & 1991 Jan. 30 & VLA:CD:1 & 8.4 & AT122 & $2.6 \times 2.1,-18$ & 0.04 & 13.3 & 17.7 & $2.1 \times 2.0$ & $3.2^{h . g}$ & 0.033 \\
\hline I19078+0901-1 & 1992 Aug. 13 & VLA:D:1 & 8.4 & AB642 & $12.1 \times 8.6,47$ & 0.03 & 3906.8 & 6884.2 & $12.7 \times 4.7$ & $12.7^{d}$ & 0.77 \\
\hline I19078+0901-2 & 1992 Aug. 13 & VLA:D:1 & 8.4 & AB642 & $12.1 \times 8.6,47$ & 0.03 & 1997.5 & 3104.3 & $8.7 \times 6.3$ & $12.7^{d}$ & 0.53 \\
\hline I19078+0901-3 & 1992 Aug. 13 & VLA:D:1 & 8.4 & AB642 & $12.1 \times 8.6,47$ & 0.03 & 665 & 2417.4 & $31.9 \times 6.9$ & $12.7^{d}$ & 1.94 \\
\hline I19097+0847-1 & 1994 Oct 04 & VLA:BC:1 & 8.4 & AM462 & $6.3 \times 2.1,63$ & 0.16 & 31.6 & 233.2 & $9.5 \times 7.9$ & $8.1^{d}$ & 0.38 \\
\hline I19097+0847-2 & 1994 Oct 04 & VLA:BC:1 & 8.4 & AM462 & $6.3 \times 2.1,63$ & 0.16 & 76.8 & 174.4 & $4.8 \times 3.2$ & $8.1^{d}$ & 0.19 \\
\hline $\mathrm{I} 19111+1048 \mathrm{~A}$ & 1997 Aug. 06 & VLA:C:1 & 8.4 & AF425 & $3.0 \times 2.7,33$ & 3.13 & 1337.3 & 3038.8 & $3.6 \times 3.2$ & $8.2^{d}$ & 0.14 \\
\hline $\mathrm{I} 19111+1048 \mathrm{~B}$ & 2003 Apr. 05 & VLA:D:1 & 8.4 & AK559 & $8.4 \times 7.6,-18$ & 2.34 & 363.1 & 422.2 & $4.4 \times 1.4$ & $7.8^{d}$ & 0.17 \\
\hline I19442+2427 & 1998 Dec 13 & VLA:C:1 & 8.4 & AK477 & $3.3 \times 2.8,36$ & 0.13 & 33.5 & 48.0 & $2.6 \times 1.4$ & $2.08^{e}$ & 0.026 \\
\hline $\mathrm{I} 20081+3122$ & 2005 Mar. 25 & VLA:B:1 & 8.4 & AS830 & $0.4 \times 0.4,45$ & 0.22 & 78.4 & 162.8 & $0.5 \times 0.0$ & $2.0^{f *}$ & 0.005 \\
\hline I23116+6111 & 1992 Aug. 13 & VLA:D:1 & 8.4 & AB642 & $8.4 \times 6.7,2.9$ & 6.96 & 417.1 & 648.2 & $8.2 \times 5.5$ & $2.4^{j}$ & 0.095 \\
\hline $\mathrm{I} 23133+6050$ & 1998 Dec 13 & VLA:C:1 & 8.4 & AK477 & $3.1 \times 1.9,69$ & 0.32 & 94.3 & 912.4 & $7.2 \times 6.8$ & $5.2^{k *}$ & 0.18 \\
\hline I $23138+5945$ & 1998 Dec 13 & VLA:C:1 & 8.4 & AK477 & $4.3 \times 2.8,75$ & 0.28 & 31.4 & 127.6 & $6.1 \times 5.8$ & $2.5^{k *}$ & 0.074 \\
\hline U49.49-0.37 & 2005 May 03 & VLA:B:1 & 4.8 & AH869 & $1.5 \times 1.3$ & 3.50 & 1173 & 6237.7 & $7.0 \times 5.5$ & $6.1^{d}$ & 0.21 \\
\hline C41.1-0.21 & 1990 Dec 07 & VLA:C:1 & 4.8 & AB573 & $15.7 \times 5.0$ & 0.31 & & & & $9^{d}$ & \\
\hline C48.93-0.29 & 1984 Jun. 09 & VLA:C:1 & 4.8 & AR104 & $5.9 \times 5.2$ & 1.84 & & & & $5.6^{d}$ & \\
\hline C49.00-0.30 & 2005 Mar. 03 & VLA:B:1 & 4.8 & AH869 & $1.3 \times 1.3$ & 1.84 & & & & $5.6^{d}$ & \\
\hline C49.38-0.30 & 1984 Jun. 09 & VLA:C:1 & 4.8 & AR104 & $5.9 \times 5.2$ & 4.03 & & & & $7.1^{d}$ & \\
\hline C51.36-0.00 & & & 4.8 & MAGPIS & $4.0 \times 4.0$ & 2.5 & & & & $6.4^{d}$ & \\
\hline C52.94-0.59 & & & 4.8 & MAGPIS & $4.0 \times 4.0$ & 2.5 & & & & $7.2^{d}$ & \\
\hline D53.64+0.24 & & & 4.8 & MAGPIS & $4.0 \times 4.0$ & 2.5 & & & & $7.7^{d}$ & \\
\hline
\end{tabular}

Notes. From left to right, the columns are (1) source name; (2) date of observation; (3) observing configuration; (4) observing frequency; (5) project code; (6) synthesized beam; (7) rms; (8) peak intensities Sp; (9) flux density Sv; (10) deconvolved size; (11) distance; (12) linear size. The linear size of the continuum source was derived from its major axis. The coordinates of the continuum sources are given in Table 2 . The intensity and size of the continuum emission of HII region was not derived as the region is extended.

References. The references from which source distances were taken are (a) Sridharan et al. (2005); (b) Sridharan et al. (2002); (c) Sakai et al. (2008); (d) Anderson \& Bania (2009); (e) Araya et al. (2002); (f) Xu et al. (2009); (g) Hou et al. (2009); (h) Heyer et al. (2001); (i) Russeil et al. (2007); (j) Reiter et al. (2011); (k) Fich \& Blitz (1984). ${ }^{(*)}$ Stands for ambiguity in distance, and so the nearest distance was used. 
X. H. Han et al.: A possible indicator for high-mass star formation sequence

Table A.3. Results of the Gaussian fit obtained from the averaged CN(1-0) emission for each clump.

\begin{tabular}{|c|c|c|c|c|c|}
\hline \multirow[t]{2}{*}{ Source } & \multirow[t]{2}{*}{ Transition } & \multirow{2}{*}{$\begin{array}{l}\text { Hyperfine } \\
\text { component }\end{array}$} & \multicolumn{3}{|c|}{$\mathrm{CN}(1-0)$} \\
\hline & & & $\begin{array}{c}V_{\mathrm{LSR}} \\
\left(\mathrm{km} \mathrm{s}^{-1}\right) \\
\end{array}$ & $\begin{array}{c}F W H M \\
\left(\mathrm{~km} \mathrm{~s}^{-1}\right)\end{array}$ & $\begin{array}{l}T_{A}^{*} \\
(\mathrm{~K}) \\
\end{array}$ \\
\hline $\mathrm{G} 22.35+0.41$ & - & - & - & - & - \\
\hline $\mathrm{G} 23.60+0.00 \mathrm{~A}$ & - & - & - & - & - \\
\hline $\mathrm{G} 23.60+0.00 \mathrm{~B}$ & - & - & - & - & - \\
\hline \multirow[t]{7}{*}{$\mathrm{G} 34.43+0.24 \mathrm{~A}$} & $J=3 / 2-1 / 2$ & $F=3 / 2-3 / 2$ & - & - & - \\
\hline & & $F=1 / 2-1 / 2$ & - & - & - \\
\hline & & $F=5 / 2-3 / 2$ & $58.82(0.26)$ & $3.94(0.55)$ & $0.10(0.03)$ \\
\hline & & $F=3 / 2-1 / 2$ & $66.41(0.35)$ & $4.46(0.99)$ & $0.08(0.03)$ \\
\hline & $J=1 / 2-1 / 2$ & $F=3 / 2-3 / 2$ & 849.9(0.44) & 4.64(0.97) & $0.06(0.03)$ \\
\hline & & $F=3 / 2-1 / 2$ & $905.2(0.24)$ & $3.48(0.56)$ & $0.09(0.03)$ \\
\hline & & $F=1 / 2-3 / 2$ & - & - & - \\
\hline \multirow[t]{7}{*}{$\mathrm{G} 34.43+0.24 \mathrm{~B}$} & $J=3 / 2-1 / 2$ & $F=3 / 2-3 / 2$ & - & - & - \\
\hline & & $F=1 / 2-1 / 2$ & - & - & - \\
\hline & & $F=5 / 2-3 / 2$ & $57.90(0.30)$ & $5.87(0.76)$ & $0.13(0.03)$ \\
\hline & & $F=3 / 2-1 / 2$ & $65.81(0.26)$ & $5.22(1.03)$ & $0.13(0.03)$ \\
\hline & $J=1 / 2-1 / 2$ & $F=3 / 2-3 / 2$ & 849.3(0.17) & $3.62(0.36)$ & $0.12(0.03)$ \\
\hline & & $F=3 / 2-1 / 2$ & 904.1(0.19) & $5.12(0.42)$ & $0.12(0.03)$ \\
\hline & & $F=1 / 2-3 / 2$ & $973.5(0.21)$ & $3.48(0.44)$ & $0.09(0.03)$ \\
\hline \multirow{7}{*}{ I18151-1208A } & $J=3 / 2-1 / 2$ & $F=3 / 2-3 / 2$ & $-16.01(0.27)$ & $4.95(0.67)$ & $0.10(0.03)$ \\
\hline & & $F=1 / 2-1 / 2$ & $8.001(0.29)$ & $4.81(0.71)$ & $0.09(0.03)$ \\
\hline & & $F=5 / 2-3 / 2$ & $31.29(0.06)$ & $3.75(0.14)$ & $0.35(0.03)$ \\
\hline & & $F=3 / 2-1 / 2$ & $38.49(0.15)$ & $3.81(0.32)$ & $0.15(0.03)$ \\
\hline & $J=1 / 2-1 / 2$ & $F=3 / 2-3 / 2$ & 822.9(0.17) & $3.84(0.38)$ & $0.12(0.03)$ \\
\hline & & $F=3 / 2-1 / 2$ & 878.0(0.19) & $3.64(0.43)$ & $0.11(0.03)$ \\
\hline & & $F=1 / 2-3 / 2$ & $947.7(0.19)$ & $3.56(0.41)$ & $0.11(0.03)$ \\
\hline \multirow[t]{7}{*}{ I18151-1208B } & $J=3 / 2-1 / 2$ & $F=3 / 2-3 / 2$ & $-14.25(0.16)$ & $3.63(0.65)$ & $0.14(0.03)$ \\
\hline & & $F=1 / 2-1 / 2$ & $10.40(0.13)$ & $2.76(0.27)$ & $0.15(0.03)$ \\
\hline & & $F=5 / 2-3 / 2$ & $33.06(0.04)$ & $2.60(0.09)$ & $0.51(0.03)$ \\
\hline & & $F=3 / 2-1 / 2$ & $40.45(0.10)$ & $2.65(0.27)$ & $0.20(0.03)$ \\
\hline & $J=1 / 2-1 / 2$ & $F=3 / 2-3 / 2$ & $824.7(0.09)$ & $2.63(0.24)$ & $0.18(0.03)$ \\
\hline & & $F=3 / 2-1 / 2$ & 879.4(0.10) & $2.67(0.23)$ & $0.17(0.03)$ \\
\hline & & $F=1 / 2-3 / 2$ & $949.2(0.10)$ & $2.47(0.22)$ & $0.16(0.03)$ \\
\hline \multirow[t]{7}{*}{ I18223-1243A } & $J=3 / 2-1 / 2$ & $F=3 / 2-3 / 2$ & - & - & - \\
\hline & & $F=1 / 2-1 / 2$ & $28.34(0.07)$ & $0.50(0.16)$ & $0.14(0.03)$ \\
\hline & & $F=5 / 2-3 / 2$ & 44.81(0.10) & $2.79(0.28)$ & $0.20(0.03)$ \\
\hline & & $F=3 / 2-1 / 2$ & $52.45(0.22)$ & $3.68(0.63)$ & $0.11(0.03)$ \\
\hline & $J=1 / 2-1 / 2$ & $F=3 / 2-3 / 2$ & 837.0(0.14) & $2.60(0.26)$ & $0.11(0.03)$ \\
\hline & & $F=3 / 2-1 / 2$ & $891.8(0.14)$ & $2.15(0.35)$ & $0.10(0.03)$ \\
\hline & & $F=1 / 2-3 / 2$ & $961.0(0.25)$ & $2.22(0.41)$ & $0.07(0.03)$ \\
\hline \multirow[t]{7}{*}{ I18223-1243B } & $J=3 / 2-1 / 2$ & $F=3 / 2-3 / 2$ & - & - & - \\
\hline & & $F=1 / 2-1 / 2$ & - & - & - \\
\hline & & $F=5 / 2-3 / 2$ & 45.01(0.09) & $3.25(0.23)$ & $0.27(0.03)$ \\
\hline & & $F=3 / 2-1 / 2$ & $52.56(0.17)$ & $2.94(0.47)$ & $0.14(0.03)$ \\
\hline & $J=1 / 2-1 / 2$ & $F=3 / 2-3 / 2$ & $836.9(0.15)$ & $2.74(0.31)$ & $0.13(0.03)$ \\
\hline & & $F=3 / 2-1 / 2$ & 892.1(0.20) & $3.63(0.65)$ & $0.12(0.03)$ \\
\hline & & $F=1 / 2-3 / 2$ & - & - & - \\
\hline \multirow[t]{7}{*}{ I18306-0835 } & $J=3 / 2-1 / 2$ & $F=3 / 2-1 / 2$ & - & - & - \\
\hline & & $F=1 / 2-1 / 2$ & - & - & - \\
\hline & & $F=5 / 2-3 / 2$ & 77.88(0.19) & $2.46(0.48)$ & $0.13(0.04)$ \\
\hline & & $F=3 / 2-1 / 2$ & - & - & - \\
\hline & $J=1 / 2-1 / 2$ & $\mathrm{~F}=3 / 2-3 / 2$ & - & - & - \\
\hline & & $F=3 / 2-1 / 2$ & - & - & - \\
\hline & & $F=1 / 2-3 / 2$ & - & - & - \\
\hline
\end{tabular}

Notes. The columns are (1) source name; (2) velocity of CN; (3) FWHM line width of CN; (4) intensity of CN. The - indicates that the averaged molecule emission of the clump is weak and that its signal-to-noise ratio is higher than 1 , but lower than 3 . 
Table A.3. continued.

\begin{tabular}{|c|c|c|c|c|c|}
\hline Source & Transition & $\begin{array}{l}\text { Hyperfine } \\
\text { component }\end{array}$ & $\begin{array}{c}V_{\mathrm{LSR}} \\
\left(\mathrm{km} \mathrm{s}^{-1}\right)\end{array}$ & $\begin{array}{c}\mathrm{CN}(1-0) \\
F W H M \\
\left(\mathrm{~km} \mathrm{~s}^{-1}\right)\end{array}$ & $\begin{array}{l}T_{A}^{*} \\
(\mathrm{~K}) \\
\end{array}$ \\
\hline \multirow{7}{*}{ I18310-0825A } & $J=3 / 2-1 / 2$ & $F=3 / 2-1 / 2$ & - & - & - \\
\hline & & $F=1 / 2-1 / 2$ & - & - & - \\
\hline & & $F=5 / 2-3 / 2$ & $85.46(0.35)$ & $3.96(0.59)$ & $0.07(0.03)$ \\
\hline & & $F=3 / 2-1 / 2$ & - & - & - \\
\hline & $J=1 / 2-1 / 2$ & $F=3 / 2-3 / 2$ & - & - & - \\
\hline & & $F=3 / 2-1 / 2$ & - & - & - \\
\hline & & $F=1 / 2-3 / 2$ & - & - & - \\
\hline \multirow{7}{*}{ I18310-0825B } & $J=3 / 2-1 / 2$ & $F=3 / 2-3 / 2$ & $37.01(0.32)$ & $3.02(0.63)$ & $0.18(0.08)$ \\
\hline & & $F=1 / 2-1 / 2$ & $63.16(2.06)$ & $5.55(3.20)$ & $0.03(0.08)$ \\
\hline & & $F=5 / 2-3 / 2$ & $84.20(0.21)$ & $4.01(0.48)$ & $0.30(0.08)$ \\
\hline & & $F=3 / 2-1 / 2$ & $91.69(0.22)$ & $2.86(0.55)$ & $0.25(0.08)$ \\
\hline & $J=1 / 2-1 / 2$ & $F=3 / 2-3 / 2$ & $875.9(0.18)$ & $1.91(0.42)$ & $0.22(0.07)$ \\
\hline & & $F=3 / 2-1 / 2$ & $931.3(0.37)$ & $3.84(0.81)$ & $0.15(0.07)$ \\
\hline & & $F=1 / 2-3 / 2$ & 1000.(0.30) & $3.06(0.77)$ & $0.17(0.07)$ \\
\hline \multirow[t]{7}{*}{ I18337-0743 } & $J=3 / 2-1 / 2$ & $F=3 / 2-1 / 2$ & - & - & - \\
\hline & & $F=1 / 2-1 / 2$ & - & - & - \\
\hline & & $F=5 / 2-3 / 2$ & $58.14(0.22)$ & $2.14(0.41)$ & $0.13(0.04)$ \\
\hline & & $F=3 / 2-1 / 2$ & - & - & - \\
\hline & $J=1 / 2-1 / 2$ & $F=3 / 2-3 / 2$ & - & - & - \\
\hline & & $F=3 / 2-1 / 2$ & - & - & - \\
\hline & & $F=1 / 2-3 / 2$ & - & - & - \\
\hline \multirow{7}{*}{ I18530+0215 } & $J=3 / 2-1 / 2$ & $F=3 / 2-1 / 2$ & - & - & - \\
\hline & & $F=1 / 2-1 / 2$ & - & - & - \\
\hline & & $F=5 / 2-3 / 2$ & 76.74(0.14) & $3.25(0.31)$ & $0.16(0.03)$ \\
\hline & & $F=3 / 2-1 / 2$ & - & - & - \\
\hline & $J=1 / 2-1 / 2$ & $F=3 / 2-3 / 2$ & $868.8(0.28)$ & $2.80(0.75)$ & $0.07(0.03)$ \\
\hline & & $F=3 / 2-1 / 2$ & $923.5(0.29)$ & $3.93(0.66)$ & $0.07(0.03)$ \\
\hline & & $F=1 / 2-3 / 2$ & $993.6(0.45)$ & $3.97(1.38)$ & $0.05(0.03)$ \\
\hline \multirow[t]{7}{*}{ I05358+3543 } & $J=3 / 2-1 / 2$ & $F=3 / 2-3 / 2$ & $-64.17(0.24)$ & $3.35(0.54)$ & $0.09(0.03)$ \\
\hline & & $F=1 / 2-1 / 2$ & $-40.33(0.27)$ & $3.01(0.60)$ & $0.08(0.03)$ \\
\hline & & $F=5 / 2-3 / 2$ & $-17.05(0.09)$ & $3.56(0.22)$ & $0.27(0.03)$ \\
\hline & & $F=3 / 2-1 / 2$ & $-9.762(0.14)$ & $2.63(0.35)$ & $0.14(0.03)$ \\
\hline & $J=1 / 2-1 / 2$ & $F=3 / 2-3 / 2$ & $774.8(0.21)$ & $3.18(0.58)$ & $0.11(0.03)$ \\
\hline & & $F=3 / 2-1 / 2$ & $829.4(0.16)$ & $2.54(0.33)$ & $0.12(0.03)$ \\
\hline & & $F=1 / 2-3 / 2$ & $899.1(0.19)$ & $3.09(0.52)$ & $0.10(0.03)$ \\
\hline \multirow{7}{*}{ I18445-0222 } & $J=3 / 2-1 / 2$ & $F=3 / 2-3 / 2$ & - & - & - \\
\hline & & $F=1 / 2-1 / 2$ & - & - & - \\
\hline & & $F=5 / 2-3 / 2$ & $87.38(0.13)$ & $3.54(0.30)$ & $0.16(0.03)$ \\
\hline & & $F=3 / 2-1 / 2$ & $94.37(0.27)$ & $3.08(0.76)$ & $0.09(0.03)$ \\
\hline & $J=1 / 2-1 / 2$ & $F=3 / 2-3 / 2$ & $878.4(0.18)$ & $1.52(0.41)$ & $0.09(0.03)$ \\
\hline & & $F=3 / 2-1 / 2$ & $933.9(0.24)$ & $2.40(0.50)$ & $0.07(0.03)$ \\
\hline & & $F=1 / 2-3 / 2$ & 1003.(0.17) & $1.73(0.40)$ & $0.09(0.03)$ \\
\hline \multirow[t]{7}{*}{ I18517+0437 } & $J=3 / 2-1 / 2$ & $F=3 / 2-3 / 2$ & - & - & - \\
\hline & & $F=1 / 2-1 / 2$ & $21.43(0.10)$ & $0.98(0.27)$ & $0.14(0.03)$ \\
\hline & & $F=5 / 2-3 / 2$ & $44.17(0.08)$ & $2.80(0.19)$ & $0.29(0.03)$ \\
\hline & & $F=3 / 2-1 / 2$ & $51.75(0.27)$ & $3.05(0.58)$ & $0.09(0.03)$ \\
\hline & $J=1 / 2-1 / 2$ & $F=3 / 2-3 / 2$ & $835.6(0.18)$ & $3.39(0.43)$ & $0.13(0.03)$ \\
\hline & & $F=3 / 2-1 / 2$ & $890.4(0.15)$ & $2.47(0.35)$ & $0.13(0.03)$ \\
\hline & & $F=1 / 2-3 / 2$ & $960.5(0.16)$ & $2.02(0.51)$ & $0.11(0.03)$ \\
\hline
\end{tabular}


Table A.3. continued.

\begin{tabular}{|c|c|c|c|c|c|}
\hline \multirow[t]{2}{*}{ Source } & \multirow[t]{2}{*}{ Transition } & \multirow{2}{*}{$\begin{array}{l}\text { Hyperfine } \\
\text { component }\end{array}$} & \multicolumn{3}{|c|}{$\mathrm{CN}(1-0)$} \\
\hline & & & $\begin{array}{c}V_{\mathrm{LSR}} \\
\left(\mathrm{km} \mathrm{s}^{-1}\right) \\
\end{array}$ & $\begin{array}{c}F W H M \\
\left(\mathrm{~km} \mathrm{~s}^{-1}\right)\end{array}$ & $\begin{array}{l}T_{\mathrm{A}}^{*} \\
(\mathrm{~K}) \\
\end{array}$ \\
\hline \multirow{7}{*}{ I20126+4104 } & \multirow[t]{4}{*}{$J=3 / 2-1 / 2$} & $F=3 / 2-3 / 2$ & $-51.20(0.09)$ & $2.77(0.28)$ & $0.23(0.03)$ \\
\hline & & $F=1 / 2-1 / 2$ & $-26.80(0.14)$ & $2.66(0.33)$ & $0.15(0.03)$ \\
\hline & & $F=5 / 2-3 / 2$ & $-3.672(0.04)$ & $2.72(0.10)$ & $0.50(0.03)$ \\
\hline & & $F=3 / 2-1 / 2$ & $3.626(0.10)$ & $2.62(0.26)$ & $0.22(0.03)$ \\
\hline & \multirow[t]{3}{*}{$J=1 / 2-1 / 2$} & $F=3 / 2-3 / 2$ & $788.0(0.08)$ & $2.58(0.25)$ & $0.23(0.03)$ \\
\hline & & $F=3 / 2-1 / 2$ & $842.9(0.08)$ & $2.57(0.23)$ & $0.22(0.03)$ \\
\hline & & $F=1 / 2-3 / 2$ & $912.3(0.10)$ & $2.47(0.26)$ & $0.17(0.03)$ \\
\hline \multirow{7}{*}{ I20293+3952 } & \multirow{4}{*}{$J=3 / 2-1 / 2$} & $F=3 / 2-3 / 2$ & $-41.34(0.13)$ & $2.21(0.30)$ & $0.14(0.03)$ \\
\hline & & $F=1 / 2-1 / 2$ & $-17.25(0.23)$ & $2.12(0.46)$ & $0.08(0.03)$ \\
\hline & & $F=5 / 2-3 / 2$ & $6.091(0.06)$ & $2.41(0.14)$ & $0.33(0.03)$ \\
\hline & & $F=1 / 2-1 / 2$ & $13.74(0.21)$ & $2.66(0.36)$ & $0.10(0.03)$ \\
\hline & \multirow[t]{3}{*}{$J=1 / 2-1 / 2$} & $F=3 / 2-3 / 2$ & $797.5(0.10)$ & $2.16(0.23)$ & $0.16(0.03)$ \\
\hline & & $F=3 / 2-1 / 2$ & $852.8(0.12)$ & $2.42(0.25)$ & $0.13(0.03)$ \\
\hline & & $F=1 / 2-3 / 2$ & $922.7(0.12)$ & $1.73(0.32)$ & $0.13(0.03)$ \\
\hline \multirow[t]{7}{*}{ I20343+4129 } & \multirow[t]{4}{*}{$J=3 / 2-1 / 2$} & $F=3 / 2-3 / 2$ & $-35.65(0.09)$ & $2.49(0.22)$ & $0.18(0.03)$ \\
\hline & & $F=1 / 2-1 / 2$ & $-11.10(0.09)$ & $1.57(0.19)$ & $0.15(0.03)$ \\
\hline & & $F=5 / 2-3 / 2$ & $11.95(0.04)$ & $2.42(0.09)$ & $0.47(0.03)$ \\
\hline & & $F=3 / 2-1 / 2$ & $19.31(0.09)$ & $2.70(0.24)$ & $0.21(0.03)$ \\
\hline & \multirow{3}{*}{$J=1 / 2-1 / 2$} & $F=3 / 2-3 / 2$ & $803.7(0.09)$ & $2.68(0.22)$ & $0.19(0.03)$ \\
\hline & & $F=3 / 2-1 / 2$ & $858.4(0.10)$ & $2.36(0.19)$ & $0.16(0.03)$ \\
\hline & & $F=1 / 2-3 / 2$ & $928.0(0.10)$ & $1.96(0.26)$ & $0.15(0.03)$ \\
\hline \multirow[t]{7}{*}{ I22134+5834 } & \multirow[t]{4}{*}{$J=3 / 2-1 / 2$} & $F=3 / 2-1 / 2$ & - & - & - \\
\hline & & $F=1 / 2-1 / 2$ & - & - & - \\
\hline & & $F=5 / 2-3 / 2$ & $-18.54(0.22)$ & $4.06(0.65)$ & $0.11(0.03)$ \\
\hline & & $F=3 / 2-1 / 2$ & - & - & - \\
\hline & \multirow[t]{3}{*}{$J=1 / 2-1 / 2$} & $F=3 / 2-3 / 2$ & - & - & - \\
\hline & & $F=3 / 2-1 / 2$ & - & - & - \\
\hline & & $F=1 / 2-3 / 2$ & - & - & - \\
\hline \multirow[t]{7}{*}{$\mathrm{I} 22551+6221$} & \multirow[t]{4}{*}{$J=3 / 2-1 / 2$} & $F=3 / 2-3 / 2$ & $-59.97(0.22)$ & $2.93(0.50)$ & $0.07(0.02)$ \\
\hline & & $F=1 / 2-1 / 2$ & - & - & - \\
\hline & & $F=5 / 2-3 / 2$ & $-12.76(0.07)$ & $2.58(0.16)$ & $0.23(0.02)$ \\
\hline & & $F=3 / 2-1 / 2$ & $-5.114(0.12)$ & $1.98(0.30)$ & $0.12(0.02)$ \\
\hline & \multirow[t]{3}{*}{$J=1 / 2-1 / 2$} & $F=3 / 2-3 / 2$ & $778.7(0.13)$ & $2.57(0.33)$ & $0.11(0.02)$ \\
\hline & & $F=3 / 2-1 / 2$ & $834.2(0.20)$ & $2.54(0.42)$ & $0.08(0.02)$ \\
\hline & & $F=1 / 2-3 / 2$ & $903.1(0.30)$ & $5.17(1.03)$ & $0.07(0.02)$ \\
\hline \multirow{7}{*}{ I22570+5912 } & \multirow{4}{*}{$J=3 / 2-1 / 2$} & $F=3 / 2-3 / 2$ & - & - & - \\
\hline & & $F=1 / 2-1 / 2$ & - & - & - \\
\hline & & $F=5 / 2-3 / 2$ & $-46.40(0.11)$ & $2.56(0.26)$ & $0.13(0.02)$ \\
\hline & & $F=3 / 2-1 / 2$ & - & - & - \\
\hline & $J=1 / 2-1 / 2$ & $F=3 / 2-3 / 2$ & - & - & - \\
\hline & & $F=3 / 2-1 / 2$ & - & - & - \\
\hline & & $F=1 / 2-3 / 2$ & - & - & - \\
\hline I23033+5951 & $J=3 / 2-1 / 2$ & $F=3 / 2-3 / 2$ & - & - & - \\
\hline & & $F=1 / 2-1 / 2$ & - & - & - \\
\hline & & $F=5 / 2-3 / 2$ & $-52.72(0.11)$ & $3.78(0.31)$ & $0.12(0.02)$ \\
\hline & & $F=3 / 2-1 / 2$ & - & - & - \\
\hline & $J=1 / 2-1 / 2$ & $F=3 / 2-3 / 2$ & - & - & - \\
\hline & & $F=3 / 2-1 / 2$ & - & - & - \\
\hline & & $F=1 / 2-3 / 2$ & - & - & - \\
\hline
\end{tabular}


Table A.3. continued.

\begin{tabular}{|c|c|c|c|c|c|}
\hline Source & Transition & $\begin{array}{l}\text { Hyperfine } \\
\text { component }\end{array}$ & $\begin{array}{c}V_{\mathrm{LSR}} \\
\left(\mathrm{km} \mathrm{s}^{-1}\right) \\
\end{array}$ & $\begin{array}{c}\mathrm{CN}(1-0) \\
F W H M \\
\left(\mathrm{~km} \mathrm{~s}^{-1}\right)\end{array}$ & $\begin{array}{l}T_{A}^{*} \\
(\mathrm{~K}) \\
\end{array}$ \\
\hline \multirow[t]{7}{*}{ I23139+5939 } & $J=3 / 2-1 / 2$ & $F=3 / 2-3 / 2$ & - & - & - \\
\hline & & $F=1 / 2-1 / 2$ & - & - & - \\
\hline & & $F=5 / 2-3 / 2$ & $-44.00(0.10)$ & $2.15(0.26)$ & $0.12(0.02)$ \\
\hline & & $F=3 / 2-1 / 2$ & - & - & - \\
\hline & $J=1 / 2-1 / 2$ & $F=3 / 2-3 / 2$ & 747.7(0.25) & $2.71(0.39)$ & $0.05(0.02)$ \\
\hline & & $F=3 / 2-1 / 2$ & - & - & - \\
\hline & & $F=1 / 2-3 / 2$ & - & - & - \\
\hline \multirow[t]{7}{*}{ I01202+6133 } & $J=3 / 2-1 / 2$ & $F=3 / 2-3 / 2$ & - & - & - \\
\hline & & $F=1 / 2-1 / 2$ & - & - & - \\
\hline & & $F=5 / 2-3 / 2$ & $-14.20(0.10)$ & $2.99(0.26)$ & $0.20(0.03)$ \\
\hline & & $F=3 / 2-1 / 2$ & $-6.625(0.22)$ & $4.04(0.56)$ & $0.10(0.03)$ \\
\hline & $J=1 / 2-1 / 2$ & $F=3 / 2-3 / 2$ & $777.4(0.18)$ & $2.55(0.36)$ & $0.09(0.03)$ \\
\hline & & $F=3 / 2-1 / 2$ & $832.7(0.22)$ & $2.76(0.45)$ & $0.08(0.03)$ \\
\hline & & $F=1 / 2-3 / 2$ & $901.6(0.23)$ & $2.15(0.42)$ & $0.07(0.03)$ \\
\hline \multirow[t]{7}{*}{ I03035+5819 } & $J=3 / 2-1 / 2$ & $F=3 / 2-3 / 2$ & $-87.21(0.35)$ & $4.66(1.05)$ & $0.08(0.03)$ \\
\hline & & $F=1 / 2-1 / 2$ & - & - & - \\
\hline & & $F=5 / 2-3 / 2$ & $-39.82(0.09)$ & $2.90(0.22)$ & $0.21(0.03)$ \\
\hline & & $F=3 / 2-1 / 2$ & $-32.31(0.06)$ & $1.01(0.17)$ & $0.20(0.03)$ \\
\hline & $J=1 / 2-1 / 2$ & $F=3 / 2-3 / 2$ & $751.9(0.14)$ & $2.69(0.62)$ & $0.14(0.03)$ \\
\hline & & $F=3 / 2-1 / 2$ & - & - & - \\
\hline & & $F=1 / 2-3 / 2$ & $876.7(0.13)$ & $2.56(0.32)$ & $0.13(0.03)$ \\
\hline \multirow[t]{7}{*}{$19078+0901$} & $J=3 / 2-1 / 2$ & $F=3 / 2-3 / 2$ & $-37.98(0.23)$ & $12.97(0.53)$ & $0.14(0.02)$ \\
\hline & & $F=1 / 2-1 / 2$ & $-13.57(0.27)$ & $14.19(0.74)$ & $0.13(0.02)$ \\
\hline & & $F=5 / 2-3 / 2$ & $8.680(0.10)$ & $14.11(0.33)$ & $0.36(0.02)$ \\
\hline & & $F=3 / 2-1 / 2$ & 20.82(0.19) & $4.38(0.39)$ & $0.12(0.02)$ \\
\hline & $J=1 / 2-1 / 2$ & $F=3 / 2-3 / 2$ & $801.2(0.25)$ & $12.91(0.55)$ & $0.13(0.02)$ \\
\hline & & $F=3 / 2-1 / 2$ & $856.7(0.27)$ & $11.58(0.70)$ & $0.12(0.02)$ \\
\hline & & $F=1 / 2-3 / 2$ & 926.3(0.29) & $10.08(0.69)$ & $0.11(0.02)$ \\
\hline \multirow[t]{7}{*}{ I19097+0847 } & $J=3 / 2-1 / 2$ & $F=3 / 2-3 / 2$ & $10.30(0.19)$ & $2.96(0.49)$ & $0.09(0.02)$ \\
\hline & & $F=1 / 2-1 / 2$ & $35.18(0.15)$ & $0.96(0.33)$ & $0.08(0.02)$ \\
\hline & & $F=5 / 2-3 / 2$ & $57.85(0.08)$ & $4.22(0.20)$ & $0.22(0.02)$ \\
\hline & & $F=3 / 2-1 / 2$ & 65.82(0.26) & $3.13(0.74)$ & $0.07(0.02)$ \\
\hline & $J=1 / 2-1 / 2$ & $F=3 / 2-3 / 2$ & 849.3(0.16) & $3.14(0.35)$ & $0.10(0.02)$ \\
\hline & & $F=3 / 2-1 / 2$ & 904.1(0.28) & $4.20(0.63)$ & $0.07(0.02)$ \\
\hline & & $F=1 / 2-3 / 2$ & $974.7(0.26)$ & $4.24(0.74)$ & $0.07(0.02)$ \\
\hline \multirow[t]{7}{*}{$\mathrm{I} 19111+1048 \mathrm{~B}$} & $J=3 / 2-1 / 2$ & $F=3 / 2-3 / 2$ & 11.54(0.40) & $4.26(0.75)$ & $0.07(0.03)$ \\
\hline & & $F=1 / 2-1 / 2$ & $35.62(0.56)$ & $6.35(1.53)$ & $0.06(0.03)$ \\
\hline & & $F=5 / 2-3 / 2$ & 58.99(0.18) & $5.31(0.43)$ & $0.16(0.03)$ \\
\hline & & $F=3 / 2-1 / 2$ & 68.62(0.32) & $2.37(0.50)$ & $0.06(0.03)$ \\
\hline & $J=1 / 2-1 / 2$ & $F=3 / 2-3 / 2$ & 849.9(0.32) & $4.82(0.72)$ & $0.09(0.03)$ \\
\hline & & $F=3 / 2-1 / 2$ & $905.9(0.40)$ & $5.97(1.18)$ & $0.08(0.03)$ \\
\hline & & $F=1 / 2-3 / 2$ & 974.2(0.46) & $4.27(0.86)$ & $0.06(0.03)$ \\
\hline \multirow[t]{7}{*}{$\mathrm{I} 19111+1048 \mathrm{~A}$} & $J=3 / 2-1 / 2$ & $F=3 / 2-3 / 2$ & $11.31(0.37)$ & $5.65(0.90)$ & $0.08(0.03)$ \\
\hline & & $F=1 / 2-1 / 2$ & $36.00(0.55)$ & $4.85(1.41)$ & $0.07(0.03)$ \\
\hline & & $F=5 / 2-3 / 2$ & $58.27(0.10)$ & $4.35(0.49)$ & $0.23(0.03)$ \\
\hline & & $F=3 / 2-1 / 2$ & 65.61(1.05) & $13.33(2.65)$ & $0.13(0.03)$ \\
\hline & $J=1 / 2-1 / 2$ & $F=3 / 2-3 / 2$ & $850.6(0.23)$ & $4.56(0.51)$ & $0.10(0.03)$ \\
\hline & & $F=3 / 2-1 / 2$ & $905.5(0.22)$ & $4.87(0.58)$ & $0.11(0.03)$ \\
\hline & & $F=1 / 2-3 / 2$ & $974.7(0.23)$ & $3.88(0.55)$ & $0.09(0.03)$ \\
\hline \multirow[t]{7}{*}{ I19442+2427 } & $J=3 / 2-1 / 2$ & $F=3 / 2-3 / 2$ & $-24.30(0.06)$ & $3.42(0.12)$ & $0.28(0.02)$ \\
\hline & & $F=1 / 2-1 / 2$ & $0.037(0.09)$ & $3.47(0.22)$ & $0.20(0.02)$ \\
\hline & & $F=5 / 2-3 / 2$ & $22.93(0.02)$ & $3.54(0.05)$ & $0.82(0.02)$ \\
\hline & & $F=3 / 2-1 / 2$ & $30.47(0.06)$ & $3.59(0.13)$ & $0.30(0.02)$ \\
\hline & $J=1 / 2-1 / 2$ & $F=3 / 2-3 / 2$ & $814.5(0.32)$ & $3.96(0.32)$ & $0.32(0.03)$ \\
\hline & & $F=3 / 2-1 / 2$ & $869.5(0.32)$ & $3.27(0.32)$ & $0.30(0.03)$ \\
\hline & & $F=1 / 2-3 / 2$ & $939.2(0.32)$ & $3.23(0.32)$ & $0.26(0.03)$ \\
\hline
\end{tabular}


Table A.3. continued.

\begin{tabular}{|c|c|c|c|c|c|}
\hline Source & Transition & $\begin{array}{l}\text { Hyperfine } \\
\text { component }\end{array}$ & $\begin{array}{c}V_{\mathrm{LSR}} \\
\left(\mathrm{km} \mathrm{s}^{-1}\right)\end{array}$ & $\begin{array}{c}\mathrm{CN}(1-0) \\
F W H M \\
\left(\mathrm{~km} \mathrm{~s}^{-1}\right)\end{array}$ & $\begin{array}{l}T_{A}^{*} \\
(\mathrm{~K}) \\
\end{array}$ \\
\hline \multirow{7}{*}{ I20081+3122 } & $J=3 / 2-1 / 2$ & $F=3 / 2-1 / 2$ & - & - & - \\
\hline & & $F=1 / 2-1 / 2$ & - & - & - \\
\hline & & $F=5 / 2-3 / 2$ & $11.22(0.28)$ & $3.82(0.51)$ & $0.16(0.06)$ \\
\hline & & $F=3 / 2-1 / 2$ & - & - & - \\
\hline & $J=1 / 2-1 / 2$ & $F=3 / 2-3 / 2$ & $858.1(0.35)$ & $4.02(0.76)$ & $0.11(0.05)$ \\
\hline & & $F=3 / 2-1 / 2$ & - & - & - \\
\hline & & $F=1 / 2-3 / 2$ & - & - & - \\
\hline \multirow{7}{*}{ I23116+6111 } & $J=3 / 2-1 / 2$ & $F=3 / 2-3 / 2$ & $-104.1(0.13)$ & $4.72(0.30)$ & $0.14(0.02)$ \\
\hline & & $F=1 / 2-1 / 2$ & $-79.64(0.18)$ & $6.47(0.69)$ & $0.10(0.02)$ \\
\hline & & $F=5 / 2-3 / 2$ & $-57.09(0.05)$ & $4.72(0.15)$ & $0.40(0.02)$ \\
\hline & & $F=3 / 2-1 / 2$ & $-49.72(0.11)$ & $4.93(0.29)$ & $0.20(0.02)$ \\
\hline & $J=1 / 2-1 / 2$ & $F=3 / 2-3 / 2$ & $734.7(0.09)$ & $5.33(0.28)$ & $0.17(0.02)$ \\
\hline & & $F=3 / 2-1 / 2$ & 789.7(0.09) & $3.99(0.23)$ & $0.15(0.02)$ \\
\hline & & $F=1 / 2-3 / 2$ & $859.3(0.11)$ & $3.78(0.25)$ & $0.12(0.02)$ \\
\hline \multirow[t]{7}{*}{$\mathrm{I} 23133+6050$} & $J=3 / 2-1 / 2$ & $F=3 / 2-3 / 2$ & $-103.2(0.22)$ & $2.48(0.47)$ & $0.12(0.04)$ \\
\hline & & $F=1 / 2-1 / 2$ & $-78.84(0.22)$ & $1.70(0.42)$ & $0.10(0.04)$ \\
\hline & & $F=5 / 2-3 / 2$ & $-56.06(0.08)$ & $3.09(0.19)$ & $0.38(0.04)$ \\
\hline & & $F=3 / 2-1 / 2$ & $-48.43(0.22)$ & $3.20(0.49)$ & $0.14(0.04)$ \\
\hline & $J=1 / 2-1 / 2$ & $F=3 / 2-3 / 2$ & $735.2(0.12)$ & $1.85(1.52)$ & $0.16(0.04)$ \\
\hline & & $F=3 / 2-1 / 2$ & $790.5(0.17)$ & $2.36(0.41)$ & $0.13(0.04)$ \\
\hline & & $F=1 / 2-3 / 2$ & - & - & - \\
\hline \multirow[t]{7}{*}{ I23138+5945 } & $J=3 / 2-1 / 2$ & $F=3 / 2-3 / 2$ & $-90.23(0.35)$ & $5.05(0.90)$ & $0.07(0.03)$ \\
\hline & & $F=1 / 2-1 / 2$ & $-65.82(0.25)$ & $3.23(0.57)$ & $0.08(0.03)$ \\
\hline & & $F=5 / 2-3 / 2$ & $-43.04(0.08)$ & $3.75(0.18)$ & $0.27(0.03)$ \\
\hline & & $F=3 / 2-1 / 2$ & $-35.64(0.17)$ & $4.66(0.53)$ & $0.14(0.03)$ \\
\hline & $J=1 / 2-1 / 2$ & $F=3 / 2-3 / 2$ & $748.8(0.14)$ & $2.66(0.34)$ & $0.12(0.03)$ \\
\hline & & $F=3 / 2-1 / 2$ & $803.8(0.17)$ & $3.38(0.54)$ & $0.12(0.03)$ \\
\hline & & $F=1 / 2-3 / 2$ & $873.4(0.20)$ & $4.21(0.58)$ & $0.11(0.03)$ \\
\hline \multirow[t]{7}{*}{ U49.49-0.37 } & $J=3 / 2-1 / 2$ & $F=3 / 2-3 / 2$ & $12.82(0.38)$ & 7.37(1.05) & $0.14(0.05)$ \\
\hline & & $F=1 / 2-1 / 2$ & $37.32(0.57)$ & $6.87(1.04)$ & $0.09(0.05)$ \\
\hline & & $F=5 / 2-3 / 2$ & $59.73(0.13)$ & $11.89(0.38)$ & $0.59(0.05)$ \\
\hline & & $F=3 / 2-1 / 2$ & 71.08(0.60) & $6.05(1.24)$ & $0.14(0.05)$ \\
\hline & $J=1 / 2-1 / 2$ & $F=3 / 2-3 / 2$ & $851.2(0.31)$ & $12.57(0.77)$ & $0.19(0.04)$ \\
\hline & & $F=3 / 2-1 / 2$ & $906.3(0.24)$ & $12.60(0.66)$ & $0.25(0.04)$ \\
\hline & & $F=1 / 2-3 / 2$ & $975.3(0.38)$ & $15.53(0.86)$ & $0.17(0.04)$ \\
\hline \multirow{7}{*}{ C41.1-0.21 } & $J=3 / 2-1 / 2$ & $F=3 / 2-1 / 2$ & - & - & - \\
\hline & & $F=1 / 2-1 / 2$ & - & - & - \\
\hline & & $F=5 / 2-3 / 2$ & $60.53(0.18)$ & $3.44(0.52)$ & $0.09(0.02)$ \\
\hline & & $F=3 / 2-1 / 2$ & - & - & - \\
\hline & $J=1 / 2-1 / 2$ & $F=3 / 2-3 / 2$ & - & - & - \\
\hline & & $F=3 / 2-1 / 2$ & - & - & - \\
\hline & & $F=1 / 2-3 / 2$ & - & - & - \\
\hline \multirow{7}{*}{ C48.93-0.29 } & $J=3 / 2-1 / 2$ & $F=3 / 2-3 / 2$ & $20.97(0.10)$ & $5.05(0.24)$ & $0.11(0.01)$ \\
\hline & & $F=1 / 2-1 / 2$ & $45.55(0.14)$ & $4.43(0.32)$ & $0.08(0.01)$ \\
\hline & & $F=5 / 2-3 / 2$ & 68.43(0.04) & $5.24(0.11)$ & $0.31(0.01)$ \\
\hline & & $F=3 / 2-1 / 2$ & 75.91(0.09) & $4.44(0.22)$ & $0.13(0.01)$ \\
\hline & $J=1 / 2-1 / 2$ & $F=3 / 2-3 / 2$ & $859.8(0.09)$ & $4.94(0.23)$ & $0.13(0.01)$ \\
\hline & & $F=3 / 2-1 / 2$ & $914.7(0.10)$ & $5.42(0.26)$ & $0.12(0.01)$ \\
\hline & & $F=1 / 2-3 / 2$ & $984.7(0.12)$ & $5.56(0.32)$ & $0.10(0.01)$ \\
\hline
\end{tabular}


Table A.3. continued.

\begin{tabular}{|c|c|c|c|c|c|}
\hline \multirow[t]{2}{*}{ Source } & \multirow[t]{2}{*}{ Transition } & \multirow{2}{*}{$\begin{array}{l}\text { Hyperfine } \\
\text { component }\end{array}$} & \multicolumn{3}{|c|}{$\mathrm{CN}(1-0)$} \\
\hline & & & $\begin{array}{c}V_{\mathrm{LSR}} \\
\left(\mathrm{km} \mathrm{s}^{-1}\right) \\
\end{array}$ & $\begin{array}{c}F W H M \\
\left(\mathrm{~km} \mathrm{~s}^{-1}\right)\end{array}$ & $\begin{array}{l}T_{A}^{*} \\
(\mathrm{~K}) \\
\end{array}$ \\
\hline \multirow{7}{*}{ C49.00-0.30 } & $J=3 / 2-1 / 2$ & $F=3 / 2-3 / 2$ & $20.46(0.14)$ & $6.06(0.39)$ & $0.11(0.02)$ \\
\hline & & $F=1 / 2-1 / 2$ & $45.15(0.15)$ & $4.40(0.44)$ & $0.09(0.02)$ \\
\hline & & $F=5 / 2-3 / 2$ & $67.63(0.10)$ & $5.21(0.17)$ & $0.35(0.02)$ \\
\hline & & $F=3 / 2-1 / 2$ & $74.12(0.24)$ & $7.16(0.48)$ & $0.19(0.02)$ \\
\hline & $J=1 / 2-1 / 2$ & $F=3 / 2-3 / 2$ & $859.9(0.14)$ & $7.65(0.32)$ & $0.13(0.02)$ \\
\hline & & $F=3 / 2-1 / 2$ & $914.6(0.14)$ & $7.85(0.32)$ & $0.13(0.02)$ \\
\hline & & $F=1 / 2-3 / 2$ & $984.5(0.14)$ & $6.49(0.36)$ & $0.12(0.02)$ \\
\hline \multirow{7}{*}{ C49.38-0.30 } & $J=3 / 2-1 / 2$ & $F=3 / 2-3 / 2$ & $3.77(0.12)$ & $4.40(0.32)$ & $0.10(0.01)$ \\
\hline & & $F=1 / 2-1 / 2$ & $28.22(0.14)$ & $3.40(0.30)$ & $0.08(0.01)$ \\
\hline & & $F=5 / 2-3 / 2$ & $51.02(0.08)$ & $4.03(0.17)$ & $0.28(0.01)$ \\
\hline & & $F=3 / 2-1 / 2$ & $58.07(0.26)$ & $8.19(1.08)$ & $0.13(0.01)$ \\
\hline & $J=1 / 2-1 / 2$ & $F=3 / 2-3 / 2$ & $842.9(0.11)$ & $4.98(0.28)$ & $0.13(0.02)$ \\
\hline & & $F=3 / 2-1 / 2$ & $897.8(0.12)$ & $5.24(0.32)$ & $0.12(0.02)$ \\
\hline & & $F=1 / 2-3 / 2$ & $967.6(0.15)$ & $5.35(0.42)$ & $0.11(0.02)$ \\
\hline \multirow{7}{*}{ C51.36-0.00 } & $J=3 / 2-1 / 2$ & $F=3 / 2-3 / 2$ & $7.36(0.21)$ & $5.71(0.57)$ & $0.08(0.02)$ \\
\hline & & $F=1 / 2-1 / 2$ & $31.55(0.38)$ & $4.33(0.89)$ & $0.05(0.02)$ \\
\hline & & $F=5 / 2-3 / 2$ & $54.31(0.08)$ & $3.91(0.20)$ & $0.18(0.02)$ \\
\hline & & $F=3 / 2-1 / 2$ & $61.75(0.16)$ & $4.44(0.46)$ & $0.09(0.02)$ \\
\hline & $J=1 / 2-1 / 2$ & $F=3 / 2-3 / 2$ & $845.9(0.19)$ & $3.78(0.52)$ & $0.07(0.02)$ \\
\hline & & $F=3 / 2-1 / 2$ & $900.9(0.16)$ & $3.74(0.45)$ & $0.07(0.02)$ \\
\hline & & $F=1 / 2-3 / 2$ & $970.4(0.15)$ & $2.84(0.36)$ & $0.07(0.02)$ \\
\hline C52.94-0.59 & - & - & - & - & - \\
\hline \multirow[t]{7}{*}{ D53.64+0.24 } & $J=3 / 2-1 / 2$ & $F=3 / 2-1 / 2$ & - & - & - \\
\hline & & $F=1 / 2-1 / 2$ & - & - & - \\
\hline & & $F=5 / 2-3 / 2$ & $36.68(0.29)$ & $3.08(0.80)$ & $0.06(0.02)$ \\
\hline & & $F=3 / 2-1 / 2$ & - & - & - \\
\hline & $J=1 / 2-1 / 2$ & $F=3 / 2-3 / 2$ & - & - & - \\
\hline & & $F=3 / 2-1 / 2$ & - & - & - \\
\hline & & $F=1 / 2-3 / 2$ & - & - & - \\
\hline
\end{tabular}


X. H. Han et al.: A possible indicator for high-mass star formation sequence

Table A.4. Results of the Gaussian fit obtained from the averaged $\mathrm{HCN}(1-0)$ emission for each clump.

\begin{tabular}{|c|c|c|c|c|}
\hline \multirow[t]{2}{*}{ Source } & \multirow{2}{*}{$\begin{array}{l}\text { Hyperfine } \\
\text { component }\end{array}$} & \multicolumn{3}{|c|}{$\mathrm{HCN}(1-0)$} \\
\hline & & $\begin{array}{c}V_{\mathrm{LSR}} \\
\left(\mathrm{km} \mathrm{s}^{-1}\right) \\
\end{array}$ & $\begin{array}{c}F W H M \\
\left(\mathrm{~km} \mathrm{~s}^{-1}\right) \\
\end{array}$ & $\begin{array}{l}T_{A}^{*} \\
(\mathrm{~K}) \\
\end{array}$ \\
\hline \multirow{3}{*}{$\mathrm{G} 22.35+0.41$} & $F=0-1$ & - & - & - \\
\hline & $F=2-1$ & $52.62(0.17)$ & $3.81(0.48)$ & $0.14(0.02)$ \\
\hline & $F=1-1$ & $57.73(0.52)$ & $4.4(1.18)$ & $0.09(0.02)$ \\
\hline \multirow[t]{3}{*}{$\mathrm{G} 23.60+0.00 \mathrm{~A}$} & $F=0-1$ & - & - & - \\
\hline & $F=2-1$ & $53.49(0.21)$ & $2.57(0.39)$ & $0.07(0.02)$ \\
\hline & $F=1-1$ & - & - & - \\
\hline \multirow[t]{3}{*}{$\mathrm{G} 23.60+0.00 \mathrm{~B}$} & $F=0-1$ & $91.48(0.84)$ & $10.3(1.62)$ & $0.07(0.02)$ \\
\hline & $F=2-1$ & 104.4(0.13) & $11.9(2.30)$ & $0.14(0.02)$ \\
\hline & $F=1-1$ & 106.2(1.19) & $12.2(2.04)$ & $0.06(0.02)$ \\
\hline \multirow[t]{3}{*}{$\mathrm{G} 34.43+0.24 \mathrm{~A}$} & $F=0-1$ & $51.16(0.18)$ & $3.07(0.41)$ & $0.09(0.02)$ \\
\hline & $F=2-1$ & $62.85(0.28)$ & $8.36(0.53)$ & $0.15(0.02)$ \\
\hline & $F=1-1$ & $63.57(0.24)$ & $2.13(0.51)$ & $0.07(0.02)$ \\
\hline \multirow[t]{3}{*}{$\mathrm{G} 34.43+0.24 \mathrm{~B}$} & $F=0-1$ & $51.68(0.97)$ & $9.53(1.54)$ & $0.11(0.02)$ \\
\hline & $F=2-1$ & 63.34(0.31) & $8.97(1.71)$ & $0.19(0.02)$ \\
\hline & $F=1-1$ & - & - & - \\
\hline \multirow[t]{3}{*}{ I18151-1208A } & $F=0-1$ & $24.18(0.19)$ & $3.88(0.41)$ & $0.09(0.02)$ \\
\hline & $F=2-1$ & $31.78(0.05)$ & $5.74(0.16)$ & $0.47(0.02)$ \\
\hline & $F=1-1$ & $36.49(0.11)$ & $3.59(0.26)$ & $0.23(0.02)$ \\
\hline \multirow{3}{*}{ I18151-1208B } & $F=0-1$ & 26.24(0.12) & $3.59(0.36)$ & $0.14(0.02)$ \\
\hline & $F=2-1$ & $33.16(0.03)$ & $3.63(0.07)$ & $0.68(0.02)$ \\
\hline & $F=1-1$ & $38.03(0.05)$ & $2.82(0.11)$ & $0.36(0.02)$ \\
\hline \multirow{3}{*}{ I18223-1243A } & $F=0-1$ & $38.18(0.17)$ & $2.53(0.37)$ & $0.08(0.02)$ \\
\hline & $F=2-1$ & 44.74(0.05) & $2.47(0.14)$ & $0.27(0.02)$ \\
\hline & $F=1-1$ & 49.83(0.09) & $2.68(0.26)$ & $0.15(0.02)$ \\
\hline \multirow[t]{3}{*}{ I18223-1243B } & $F=0-1$ & 38.84(0.38) & $4.53(0.97)$ & $0.07(0.02)$ \\
\hline & $F=2-1$ & 44.91(0.05) & $2.21(0.09)$ & $0.35(0.02)$ \\
\hline & $F=1-1$ & 49.93(0.12) & $4.41(0.28)$ & $0.19(0.02)$ \\
\hline \multirow[t]{3}{*}{ I18306-0835 } & $F=0-1$ & - & - & - \\
\hline & $F=2-1$ & 77.49(0.15) & $2.45(0.38)$ & $0.10(0.03)$ \\
\hline & $F=1-1$ & 81.93(0.49) & 10.57(1.39) & $0.12(0.03)$ \\
\hline \multirow{3}{*}{ I18310-0825A } & $F=0-1$ & 78.82(0.28) & $3.26(0.81)$ & $0.07(0.02)$ \\
\hline & $F=2-1$ & $88.70(0.81)$ & $15.23(2.16)$ & $0.08(0.02)$ \\
\hline & $F=1-1$ & 106.2(0.63) & $12.69(1.38)$ & $0.09(0.02)$ \\
\hline \multirow[t]{3}{*}{ I18310-0825B } & $F=0-1$ & 77.87(0.40) & $3.62(0.68)$ & $0.06(0.03)$ \\
\hline & $F=2-1$ & 84.02(0.21) & $3.40(0.41)$ & $0.11(0.03)$ \\
\hline & $F=1-1$ & 89.47(0.29) & $2.18(0.66)$ & $0.06(0.03)$ \\
\hline \multirow[t]{3}{*}{ I18337-0743 } & $F=0-1$ & $56.28(0.07)$ & $0.45(0.09)$ & $0.15(0.03)$ \\
\hline & $F=2-1$ & $62.38(0.71)$ & $5.57(0.89)$ & $0.09(0.04)$ \\
\hline & $F=1-1$ & $63.80(0.46)$ & $3.80(0.88)$ & $0.09(0.03)$ \\
\hline \multirow[t]{3}{*}{ I18530+0215 } & $F=0-1$ & - & - & - \\
\hline & $F=2-1$ & 77.01(0.07) & $3.67(0.18)$ & $0.20(0.02)$ \\
\hline & $F=1-1$ & - & - & - \\
\hline \multirow[t]{3}{*}{ I05358+3543 } & $F=0-1$ & $-24.23(0.10)$ & $3.34(0.25)$ & $0.17(0.02)$ \\
\hline & $F=2-1$ & $-17.41(0.03)$ & $3.41(0.09)$ & $0.62(0.02)$ \\
\hline & $F=1-1$ & $-12.47(0.08)$ & $4.00(0.25)$ & $0.30(0.02)$ \\
\hline \multirow{3}{*}{ I18445-0222 } & $F=0-1$ & - & - & - \\
\hline & $F=2-1$ & 87.37(0.09) & $3.84(0.22)$ & $0.28(0.03)$ \\
\hline & $F=1-1$ & $91.59(0.16)$ & $2.85(0.35)$ & $0.14(0.02)$ \\
\hline I18517+0437 & $F=0-1$ & $37.28(0.18)$ & $2.59(0.49)$ & $0.09(0.02)$ \\
\hline & $F=2-1$ & 44.12(0.04) & $3.22(0.09)$ & $0.56(0.02)$ \\
\hline & $F=1-1$ & 49.02(0.06) & $2.97(0.16)$ & $0.31(0.02)$ \\
\hline I $20126+4104$ & $F=0-1$ & $-10.66(0.16)$ & $4.20(0.38)$ & $0.16(0.03)$ \\
\hline & $F=2-1$ & $-3.76(0.04)$ & $2.78(0.10)$ & $0.68(0.03)$ \\
\hline & $F=1-1$ & $1.011(0.08)$ & $4.10(0.29)$ & $0.36(0.03)$ \\
\hline
\end{tabular}


Table A.4. continued.

\begin{tabular}{|c|c|c|c|c|}
\hline \multirow[t]{2}{*}{ Source } & \multirow{2}{*}{$\begin{array}{l}\text { Hyperfine } \\
\text { component }\end{array}$} & \multicolumn{3}{|c|}{$\mathrm{HCN}(1-0)$} \\
\hline & & $\begin{array}{c}V_{\mathrm{LSR}} \\
\left(\mathrm{km} \mathrm{s}^{-1}\right)\end{array}$ & $\begin{array}{c}F W H M \\
\left(\mathrm{~km} \mathrm{~s}^{-1}\right)\end{array}$ & $\begin{array}{l}T_{A}^{*} \\
(\mathrm{~K})\end{array}$ \\
\hline \multirow[t]{3}{*}{ I20293+3952 } & $F=0-1$ & $-1.07(0.09)$ & $2.37(0.26)$ & $0.15(0.02)$ \\
\hline & $F=2-1$ & $6.27(0.06)$ & $3.04(0.17)$ & $0.39(0.03)$ \\
\hline & $F=1-1$ & $11.07(0.13)$ & $3.54(0.34)$ & $0.15(0.02)$ \\
\hline \multirow{3}{*}{ I $20343+4129$} & $F=0-1$ & $5.10(0.33)$ & $1.71(0.61)$ & $0.17(0.08)$ \\
\hline & $F=2-1$ & $11.75(0.09)$ & $2.64(0.20)$ & $0.74(0.09)$ \\
\hline & $F=1-1$ & $16.50(0.13)$ & $2.167(0.28)$ & $0.41(0.08)$ \\
\hline \multirow[t]{3}{*}{ I22134+5834 } & $F=0-1$ & $-25.69(0.12)$ & $1.34(0.24)$ & $0.07(0.01)$ \\
\hline & $F=2-1$ & $-18.40(0.06)$ & $2.89(0.15)$ & $0.17(0.01)$ \\
\hline & $F=1-1$ & $-13.91(0.10)$ & $2.23(0.24)$ & $0.09(0.01)$ \\
\hline \multirow[t]{3}{*}{$\mathrm{I} 22551+6221$} & $F=0-1$ & $-19.63(0.10)$ & $1.25(0.27)$ & $0.10(0.02)$ \\
\hline & $F=2-1$ & $-12.86(0.06)$ & $2.66(0.12)$ & $0.26(0.02)$ \\
\hline & $F=1-1$ & $-7.936(0.11)$ & $2.67(0.25)$ & $0.13(0.02)$ \\
\hline \multirow[t]{3}{*}{ I $22570+5912$} & $F=0-1$ & $-54.13(0.23)$ & $3.36(0.59)$ & $0.06(0.02)$ \\
\hline & $F=2-1$ & $-46.80(0.09)$ & $3.49(0.22)$ & $0.17(0.02)$ \\
\hline & $F=1-1$ & $-42.01(0.13)$ & $2.93(0.28)$ & $0.12(0.02)$ \\
\hline \multirow[t]{3}{*}{ I23033+5951 } & $F=0-1$ & $-59.64(0.21)$ & $2.87(0.33)$ & $0.09(0.02)$ \\
\hline & $F=2-1$ & $-52.88(0.09)$ & $3.36(0.20)$ & $0.30(0.02)$ \\
\hline & $F=1-1$ & $-47.79(0.16)$ & $3.77(0.43)$ & $0.17(0.02)$ \\
\hline \multirow{3}{*}{ I23139+5939 } & $F=0-1$ & - & - & - \\
\hline & $F=2-1$ & $-43.85(0.11)$ & $2.53(0.29)$ & $0.18(0.02)$ \\
\hline & $F=1-1$ & - & - & - \\
\hline \multirow[t]{3}{*}{ I01202+6133 } & $F=0-1$ & $-21.03(0.14)$ & $2.41(0.33)$ & $0.11(0.02)$ \\
\hline & $F=2-1$ & $-14.21(0.03)$ & $2.22(0.08)$ & $0.62(0.03)$ \\
\hline & $F=1-1$ & $-9.21(0.06)$ & $2.22(0.15)$ & $0.23(0.02)$ \\
\hline \multirow[t]{3}{*}{ I03035+5819 } & $F=0-1$ & $-46.89(0.17)$ & $2.11(0.44)$ & $0.09(0.02)$ \\
\hline & $F=2-1$ & $-39.77(0.03)$ & $2.47(0.08)$ & $0.44(0.02)$ \\
\hline & $F=1-1$ & $-34.87(0.07)$ & $2.39(0.17)$ & $0.23(0.02)$ \\
\hline \multirow[t]{3}{*}{ I19078+0901 } & $F=0-1$ & $4.82(0.38)$ & $14.01(0.75)$ & $0.26(0.02)$ \\
\hline & $F=2-1$ & $7.89(0.11)$ & $17.32(0.25)$ & $0.32(0.02)$ \\
\hline & $F=1-1$ & $16.33(0.79)$ & $7.87(0.98)$ & $0.09(0.02)$ \\
\hline \multirow{3}{*}{ I19097+0847 } & $F=0-1$ & $50.77(0.13)$ & $3.28(0.25)$ & $0.10(0.01)$ \\
\hline & $F=2-1$ & $57.61(0.08)$ & $3.97(0.28)$ & $0.21(0.01)$ \\
\hline & $F=1-1$ & $61.51(0.89)$ & $8.73(1.18)$ & $0.08(0.01)$ \\
\hline \multirow{3}{*}{ I19111+1048B } & $F=0-1$ & $103.0(1.34)$ & $3.08(1.86)$ & $0.03(0.04)$ \\
\hline & $F=2-1$ & $59.14(0.05)$ & $3.27(0.40)$ & $0.16(0.04)$ \\
\hline & $F=1-1$ & $62.84(0.23)$ & $1.81(0.40)$ & $0.08(0.04)$ \\
\hline \multirow[t]{3}{*}{$\mathrm{I} 19111+1048 \mathrm{~A}$} & $F=0-1$ & $50.98(0.29)$ & $3.56(0.67)$ & $0.12(0.04)$ \\
\hline & $F=2-1$ & $59.87(0.17)$ & $5.12(0.34)$ & $0.36(0.04)$ \\
\hline & $F=1-1$ & $61.28(0.44)$ & $5.29(0.79)$ & $0.29(0.04)$ \\
\hline \multirow[t]{3}{*}{ I19442+2427 } & $F=0-1$ & $15.57(0.07)$ & $3.34(0.16)$ & $0.23(0.02)$ \\
\hline & $F=2-1$ & $22.96(0.02)$ & $3.95(0.05)$ & $1.18(0.02)$ \\
\hline & $F=1-1$ & $27.87(0.04)$ & $3.45(0.10)$ & $0.55(0.02)$ \\
\hline \multirow[t]{3}{*}{$\mathrm{I} 20081+3122$} & $F=0-1$ & $4.55(0.22)$ & $4.18(0.49)$ & $0.09(0.02)$ \\
\hline & $F=2-1$ & $12.87(0.20)$ & $5.34(0.95)$ & $0.16(0.02)$ \\
\hline & $F=1-1$ & $10.12(0.17)$ & $2.78(0.49)$ & $0.11(0.02)$ \\
\hline \multirow[t]{3}{*}{ I23116+6111 } & $F=0-1$ & $-64.04(0.04)$ & $4.69(0.11)$ & $0.35(0.01)$ \\
\hline & $F=2-1$ & $-57.72(0.02)$ & $3.88(0.08)$ & $0.71(0.01)$ \\
\hline & $F=1-1$ & $-52.86(0.20)$ & $8.25(0.34)$ & $0.36(0.01)$ \\
\hline $\mathrm{I} 23133+6050$ & $F=0-1$ & $-63.37(0.11)$ & $2.61(0.26)$ & $0.13(0.02)$ \\
\hline & $F=2-1$ & $-56.25(0.03)$ & $3.05(0.08)$ & $0.61(0.02)$ \\
\hline & $F=1-1$ & $-51.35(0.08)$ & $3.57(0.20)$ & $0.26(0.02)$ \\
\hline $\mathrm{I} 23138+5945$ & $F=0-1$ & $-50.39(0.18)$ & $5.38(0.61)$ & $0.11(0.02)$ \\
\hline & $F=2-1$ & $-43.18(0.04)$ & $3.72(0.11)$ & $0.52(0.02)$ \\
\hline & $F=1-1$ & $-38.00(0.08)$ & $3.50(0.22)$ & $0.24(0.02)$ \\
\hline
\end{tabular}


Table A.4. continued.

\begin{tabular}{lcccc}
\hline \hline Source & $\begin{array}{c}\text { Hyperfine } \\
\text { component }\end{array}$ & $\begin{array}{c}V_{\mathrm{LSR}} \\
\left(\mathrm{km} \mathrm{s}^{-1}\right)\end{array}$ & $\begin{array}{c}\mathrm{HCN}(1-0) \\
F W H M \\
\left(\mathrm{~km} \mathrm{~s}^{-1}\right)\end{array}$ & $\begin{array}{c}T_{A}^{*} \\
(\mathrm{~K})\end{array}$ \\
\hline U49.49-0.37 & $F=0-1$ & $55.87(0.17)$ & $15.04(0.33)$ & $0.74(0.03)$ \\
& $F=2-1$ & $62.00(0.08)$ & $9.12(0.18)$ & $0.80(0.03)$ \\
C41.1-0.21 & $F=1-1$ & $70.21(0.36)$ & $10.16(0.71)$ & $0.18(0.03)$ \\
& $F=0-1$ & $52.73(0.35)$ & $4.58(0.78)$ & $0.05(0.01)$ \\
& $F=2-1$ & $60.48(0.14)$ & $4.46(0.45)$ & $0.13(0.01)$ \\
C48.93-0.29 & $F=1-1$ & $65.10(0.20)$ & $2.78(0.56)$ & $0.08(0.01)$ \\
& $F=0-1$ & $62.09(0.37)$ & $6.65(0.92)$ & $0.08(0.01)$ \\
C49.00-0.30 & $F=1-1$ & $67.81(0.06)$ & $3.36(0.22)$ & $0.31(0.01)$ \\
& $F=0-1$ & $60.70(0.29)$ & $5.11(0.72)$ & $0.13(0.02)$ \\
& $F=2-1$ & $68.38(0.22)$ & $6.29(0.49)$ & $0.48(0.02)$ \\
C49.38-0.30 & $F=1-1$ & $72.39(0.40)$ & $6.68(0.50)$ & $0.37(0.02)$ \\
& $F=0-1$ & $43.97(0.19)$ & $3.90(0.39)$ & $0.08(0.02)$ \\
& $F=2-1$ & $51.31(0.09)$ & $5.33(0.22)$ & $0.39(0.02)$ \\
C51.36-0.00 & $F=1-1$ & $58.50(0.27)$ & $6.03(0.57)$ & $0.14(0.02)$ \\
& $F=0-1$ & $47.20(0.14)$ & $3.52(0.29)$ & $0.08(0.01)$ \\
& $F=2-1$ & $53.64(0.06)$ & $3.15(0.13)$ & $0.26(0.01)$ \\
C52.94-0.59 & $F=1-1$ & $59.00(0.13)$ & $5.01(0.36)$ & $0.14(0.01)$ \\
& $F=0-1$ & - & - & - \\
& $F=2-1$ & $47.54(0.33)$ & $5.33(1.39)$ & $0.07(0.02)$ \\
D53.64+0.24 & $F=0-1$ & - & - & - \\
\hline & - & - & - & - \\
\hline & & & &
\end{tabular}


Table A.5. Results of the Gaussian fit obtained from the averaged HNC(1-0) emission for each clump.

\begin{tabular}{|c|c|c|c|}
\hline \multirow[t]{2}{*}{ Source } & \multicolumn{3}{|c|}{$\operatorname{HNC}(1-0)$} \\
\hline & $\begin{array}{c}V_{\mathrm{LSR}} \\
\left(\mathrm{km} \mathrm{s}^{-1}\right)\end{array}$ & $\begin{array}{c}F W H M \\
\left(\mathrm{~km} \mathrm{~s}^{-1}\right)\end{array}$ & $\begin{array}{l}T_{A}^{*} \\
(\mathrm{~K})\end{array}$ \\
\hline $\mathrm{G} 22.35+0.41$ & $52.72(0.19)$ & $2.76(0.52)$ & $0.11(0.03)$ \\
\hline $\mathrm{G} 23.60+0.00 \mathrm{~A}$ & $53.54(0.09)$ & $2.87(0.21)$ & $0.22(0.03)$ \\
\hline $\mathrm{G} 23.60+0.00 \mathrm{~B}$ & $104.80(0.19)$ & $4.77(0.52)$ & $0.13(0.03)$ \\
\hline $\mathrm{G} 34.43+0.24 \mathrm{~A}$ & $58.82(0.06)$ & $3.35(0.14)$ & $0.39(0.03)$ \\
\hline $\mathrm{G} 34.43+0.24 \mathrm{~B}$ & $57.87(0.06)$ & $4.63(0.15)$ & $0.50(0.03)$ \\
\hline I18151-1208A & $31.15(0.09)$ & $4.23(0.21)$ & $0.29(0.03)$ \\
\hline I18151-1208B & $33.09(0.06)$ & $2.87(0.17)$ & $0.39(0.03)$ \\
\hline I18223-1243A & $45.08(0.06)$ & $2.79(0.15)$ & $0.32(0.02)$ \\
\hline I18223-1243B & $45.33(0.05)$ & $2.51(0.12)$ & $0.39(0.03)$ \\
\hline I18306-0835 & 77.89(0.08) & $3.62(0.20)$ & $0.26(0.03)$ \\
\hline I18310-0825A & 86.54(0.16) & $4.78(0.34)$ & $0.15(0.02)$ \\
\hline I18310-0825B & $84.61(0.14)$ & $3.22(0.35)$ & $0.19(0.03)$ \\
\hline I18337-0743 & $58.47(0.15)$ & $4.27(0.31)$ & $0.31(0.05)$ \\
\hline I18530+0215 & $76.92(0.07)$ & $4.15(0.18)$ & $0.29(0.02)$ \\
\hline I05358+3543 & $-17.03(0.05)$ & $3.33(0.11)$ & $0.55(0.03)$ \\
\hline I18445-0222 & $87.10(0.11)$ & $3.30(0.26)$ & $0.21(0.03)$ \\
\hline $\mathrm{I} 18517+0437$ & $44.18(0.04)$ & $2.82(0.10)$ & $0.35(0.02)$ \\
\hline I20126+4104 & $-3.61(0.04)$ & $2.82(0.09)$ & $0.61(0.03)$ \\
\hline I20293+3952 & $6.08(0.03)$ & $2.58(0.08)$ & $0.57(0.02)$ \\
\hline I20343+4129 & $11.64(0.04)$ & $2.52(0.09)$ & $0.45(0.03)$ \\
\hline I22134+5834 & $-18.46(0.08)$ & $2.08(0.20)$ & $0.18(0.02)$ \\
\hline I $22551+6221$ & $-12.78(0.12)$ & $2.73(0.25)$ & $0.14(0.02)$ \\
\hline I22570+5912 & $-46.84(0.19)$ & $3.07(0.45)$ & $0.10(0.03)$ \\
\hline I23033+5951 & $-52.69(0.08)$ & $3.49(0.21)$ & $0.27(0.03)$ \\
\hline I23139+5939 & $-43.26(0.18)$ & $3.26(0.48)$ & $0.12(0.03)$ \\
\hline I01202+6133 & $-13.94(0.04)$ & $2.29(0.10)$ & $0.37(0.02)$ \\
\hline I03035+5819 & $-39.89(0.06)$ & $2.19(0.14)$ & $0.31(0.03)$ \\
\hline I19078+0901 & $8.93(0.16)$ & $12.36(0.35)$ & $0.19(0.02)$ \\
\hline I19097+0847 & $57.63(0.10)$ & $3.74(0.24)$ & $0.27(0.03)$ \\
\hline I19111+1048B & $58.90(0.23)$ & $5.22(0.60)$ & $0.18(0.04)$ \\
\hline $\mathrm{I} 19111+1048 \mathrm{~A}$ & $58.70(0.11)$ & $5.20(0.29)$ & $0.29(0.03)$ \\
\hline I19442+2427 & 23.04(0.03) & $3.66(0.08)$ & $0.68(0.03)$ \\
\hline $\mathrm{I} 20081+3122$ & $11.33(0.06)$ & $4.53(0.15)$ & $0.35(0.02)$ \\
\hline I23116+6111 & $-57.00(0.03)$ & $4.93(0.08)$ & $0.62(0.02)$ \\
\hline $\mathrm{I} 23133+6050$ & $-56.33(0.08)$ & $3.37(0.19)$ & $0.28(0.03)$ \\
\hline I23138+5945 & $-42.94(0.10)$ & $3.48(0.26)$ & $0.24(0.03)$ \\
\hline U49.49-0.37 & $59.88(0.11)$ & $15.03(0.26)$ & $0.62(0.04)$ \\
\hline C41.1-0.21 & 60.34(0.15) & 4.03(0.39) & $0.10(0.02)$ \\
\hline C48.93-0.29 & 68.35(0.09) & $4.25(0.22)$ & $0.28(0.03)$ \\
\hline C49.00-0.30 & 68.03(0.09) & $6.34(0.25)$ & $0.35(0.03)$ \\
\hline C49.38-0.30 & 51.15(0.09) & $5.28(0.24)$ & $0.27(0.03)$ \\
\hline C51.36-0.00 & $54.36(0.09)$ & $3.72(0.24)$ & $0.23(0.02)$ \\
\hline C52.94-0.59 & $47.09(0.47)$ & $5.07(0.82)$ & $0.05(0.02)$ \\
\hline D53.64+0.24 & $37.42(0.31)$ & $5.22(0.56)$ & $0.08(0.02)$ \\
\hline
\end{tabular}

Notes. The columns are (1) source name; (2) velocity of HCN; (3) FWHM line width of HCN; (4) intensity of HCN. The - indicates that the averaged molecule emission of the clump is weak and that its signal-to-noise ratio is higher than 1, but lower than 3 . 
X. H. Han et al.: A possible indicator for high-mass star formation sequence

\section{Appendix B: $\mathrm{CN}(1-0), \mathrm{HCN}(1-0)$ and $\mathrm{HNC}(1-0)$ maps of all clumps}
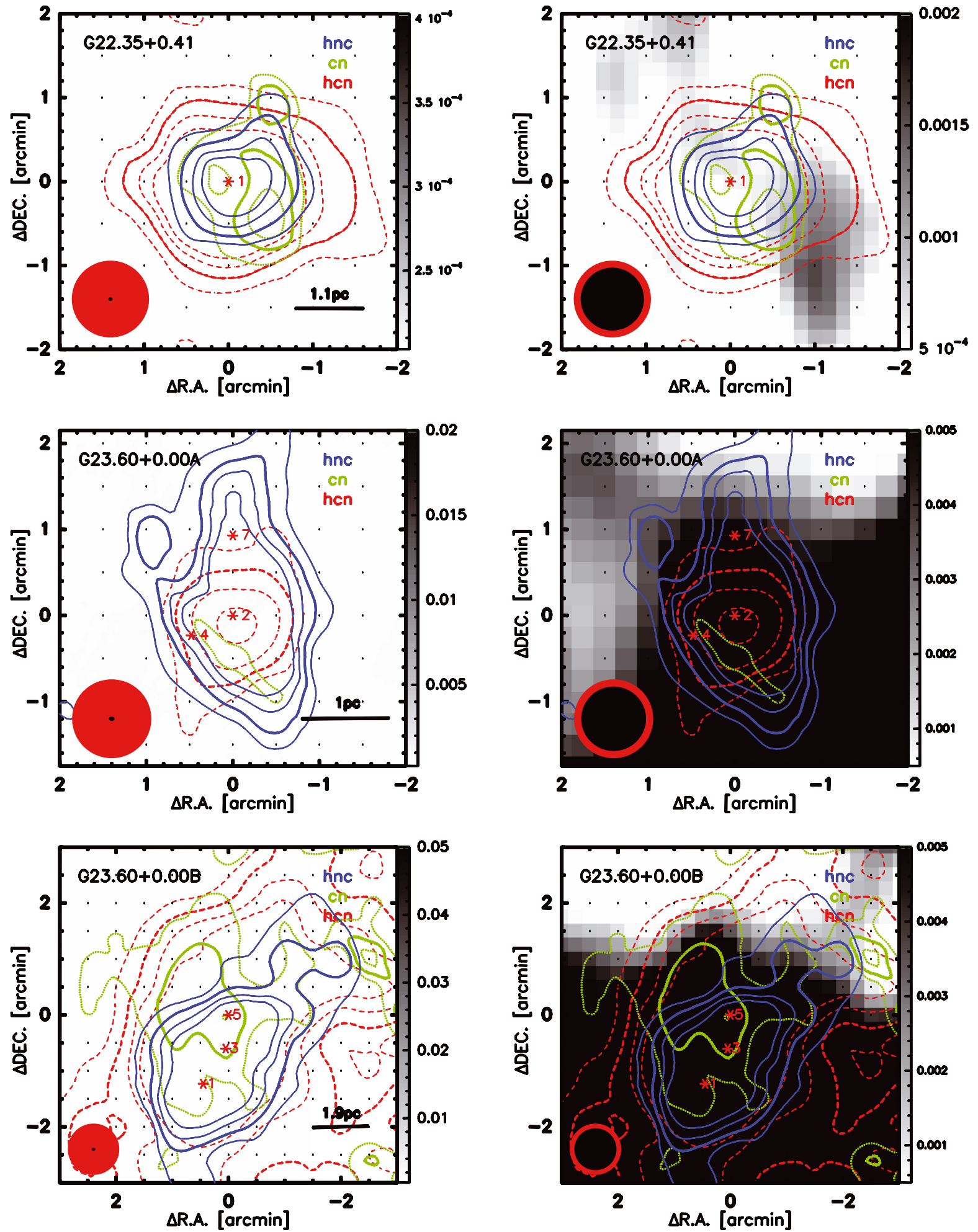

Fig. B.1. Contour maps for the integrated intensity of CN 1-0 (green), HCN (red) and HNC 1-0 (blue) from each clump superimposed on their respective continuum map in gray scale at 8.4 (or 4.8) GHz (left) and $1.4 \mathrm{GHz}$ continuum map (right). The contour levels are 30\%, 50\%, 70\%, and $90 \%$ of the map peak. The heavy lines represent $50 \%$ of the map peak. Here $\mathrm{CN}(1-0), \mathrm{HCN}(1-0)$, and $\mathrm{HNC}(1-0)$ emission of G22.35+0.41, G23.60+0.00A, G23.60+0.00B, I18310-0825A and I18310-0825B is very weak, the corresponding contour levels are $2 \sigma, 3 \sigma$ (heavy line), $4 \sigma$ and $5 \sigma$, respectively. The red asterisk in the map indicates the position of HMSC, HMPO, UCHII and HII regions. The red circle in the bottom left corner represents the FWHM beam size for molecular lines, the enclosed black circle indicates continuum emission observations. The short line in the bottom right corner of the left panel indicates the linear scale corresponding to one arcminute at the distance of the clump. The unit of the gray scale bar on the right is in Jy/beam. 
A\&A 576, A131 (2015)
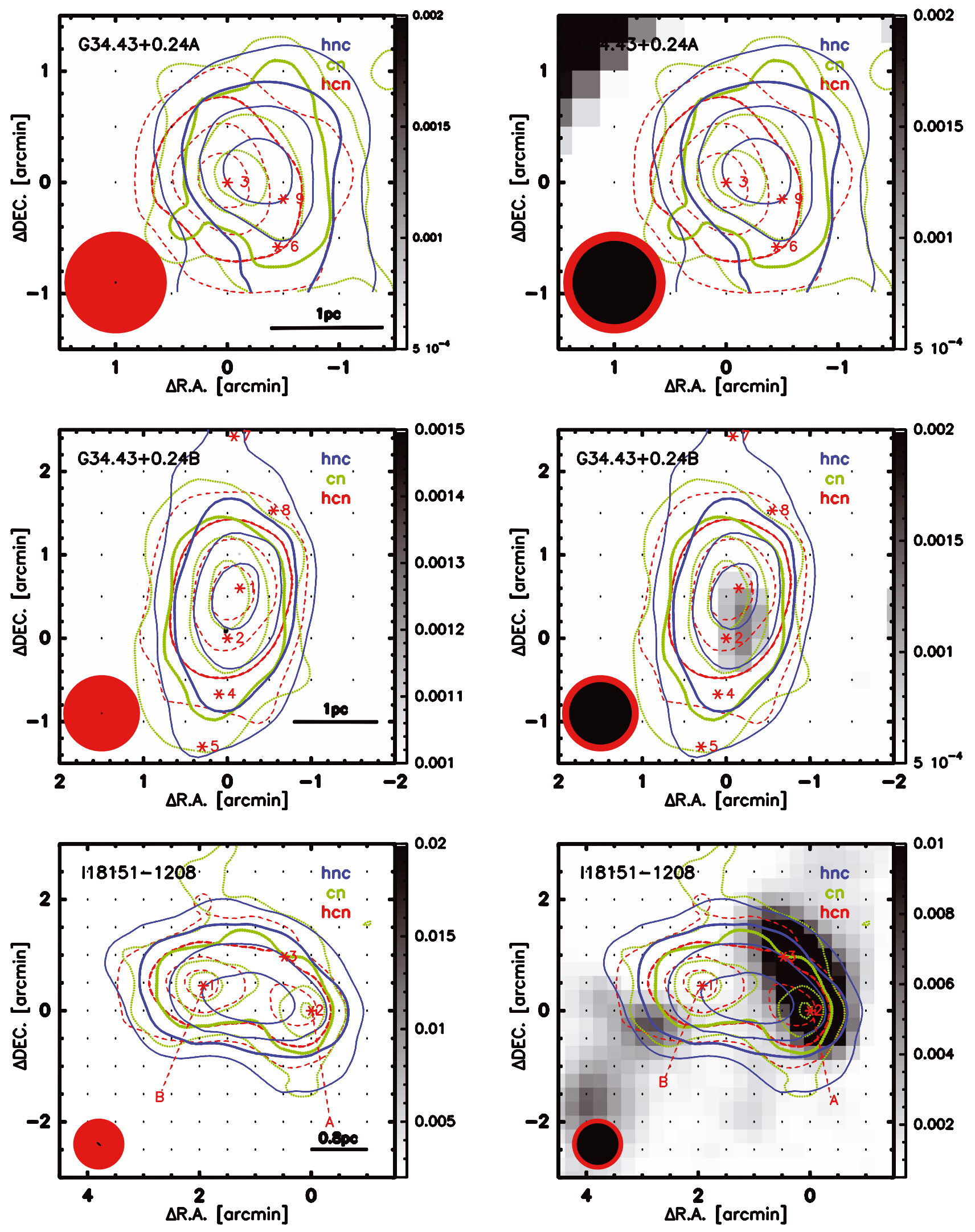

Fig. B.1. continued. 
X. H. Han et al.: A possible indicator for high-mass star formation sequence
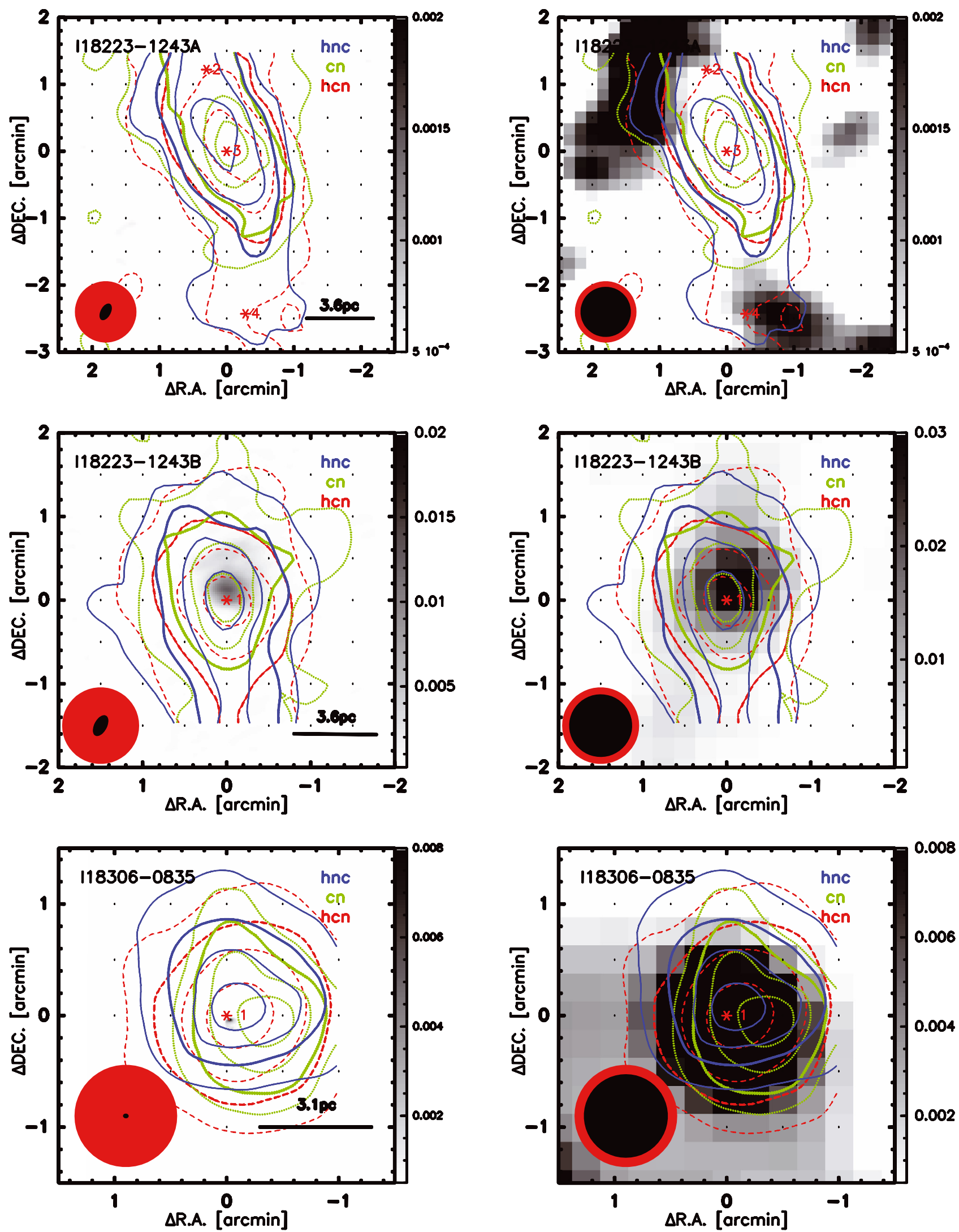

Fig. B.1. continued. 

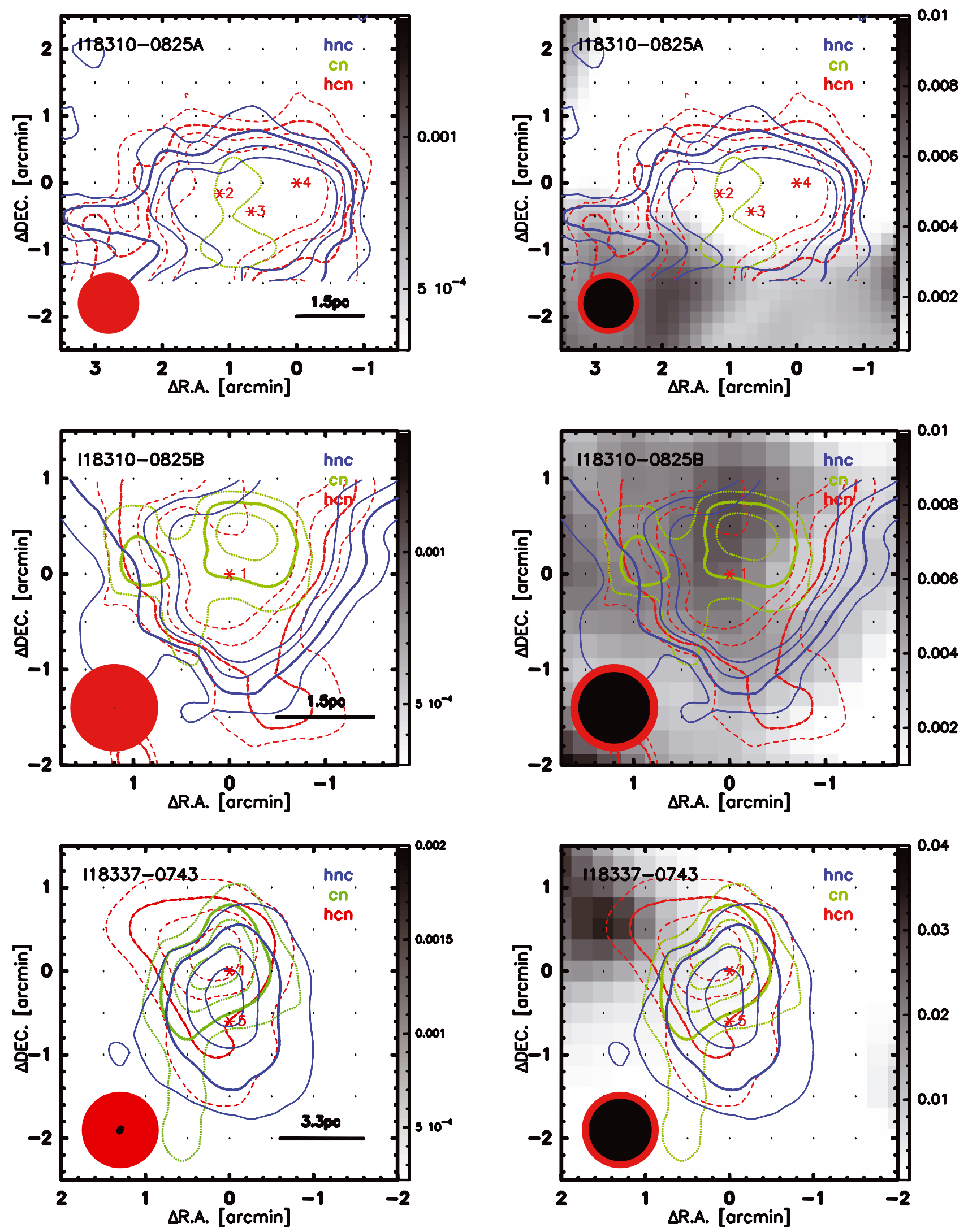

Fig. B.1. continued. 
X. H. Han et al.: A possible indicator for high-mass star formation sequence
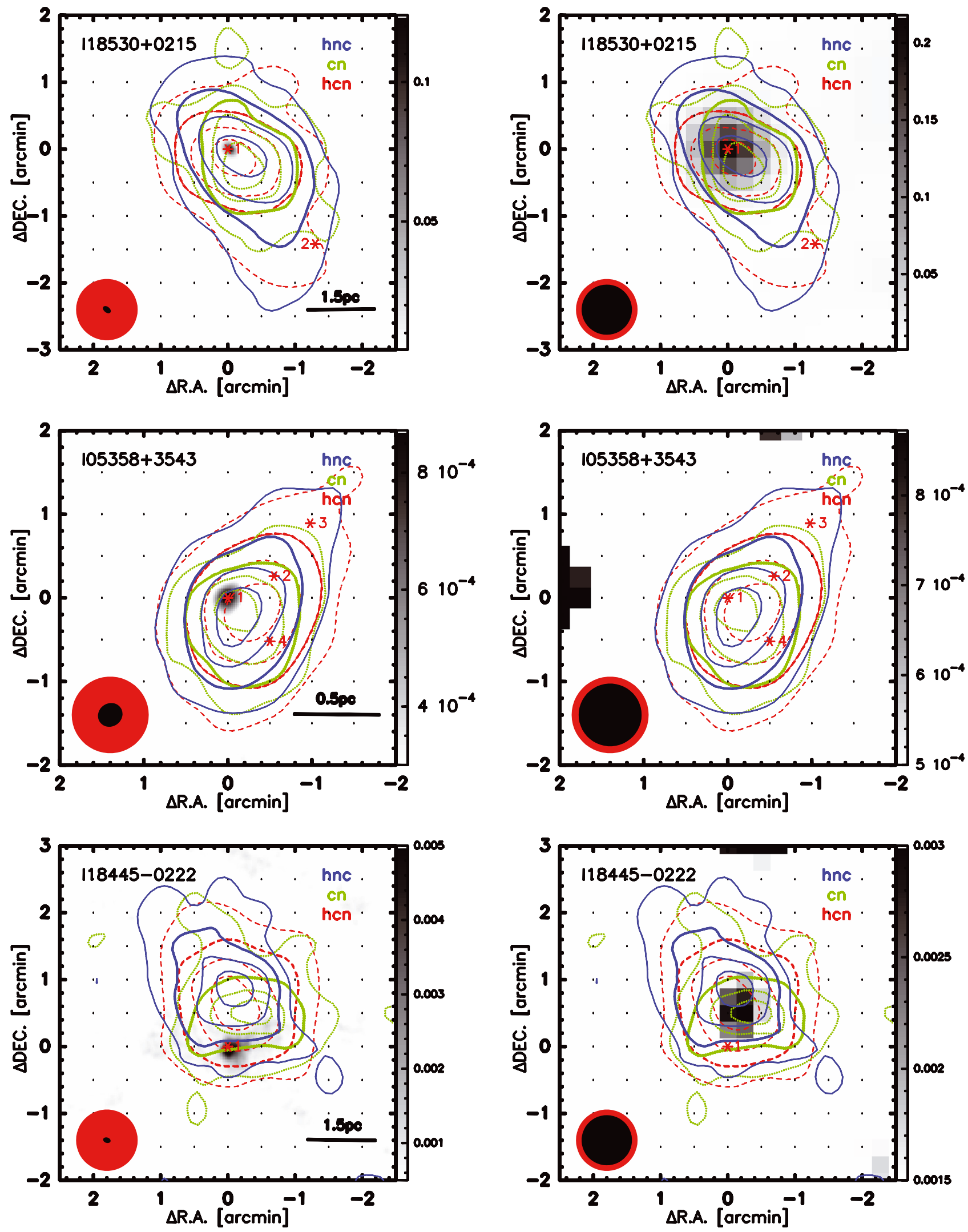

Fig. B.1. continued. 
A\&A 576, A131 (2015)
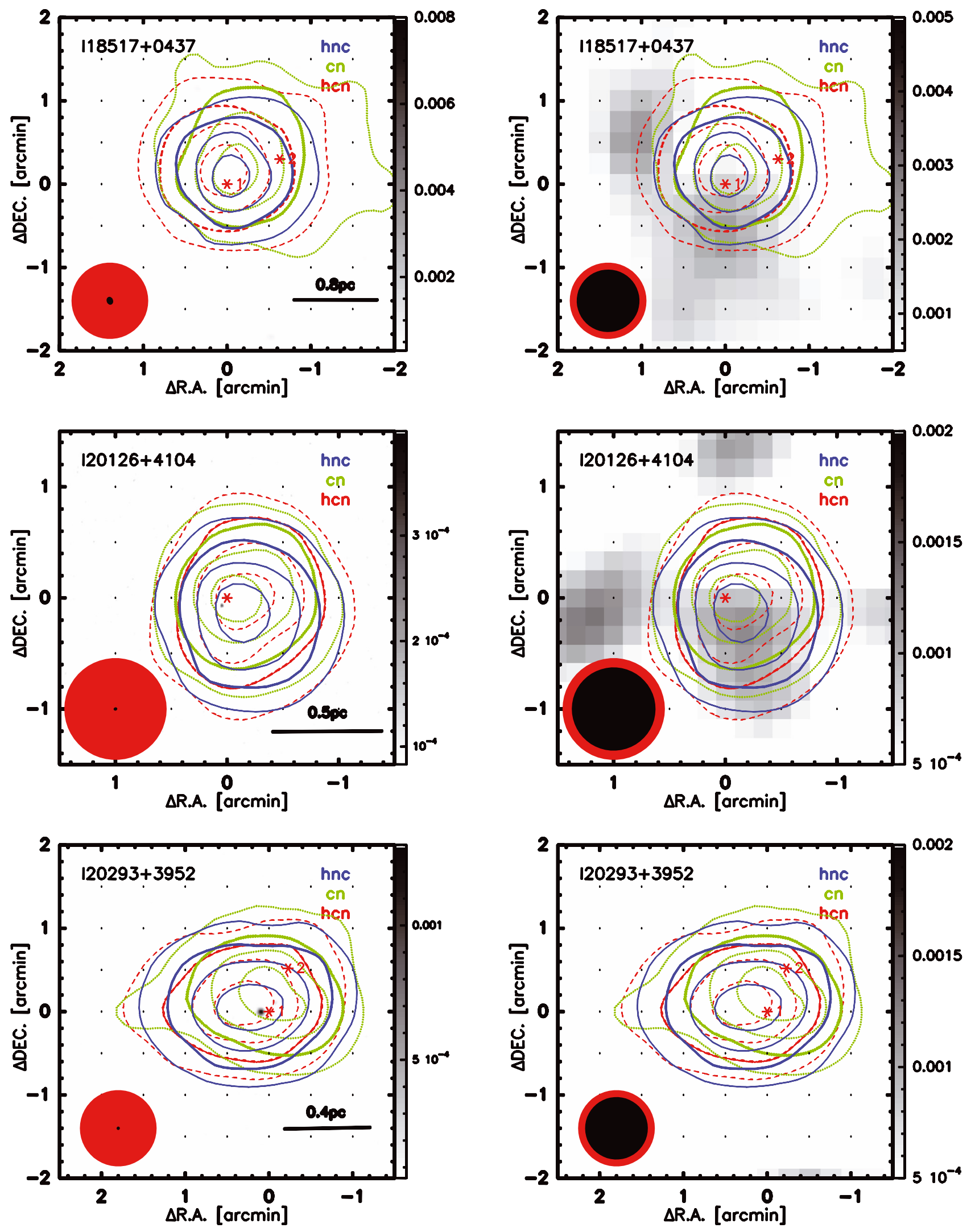

Fig. B.1. continued. 
X. H. Han et al.: A possible indicator for high-mass star formation sequence
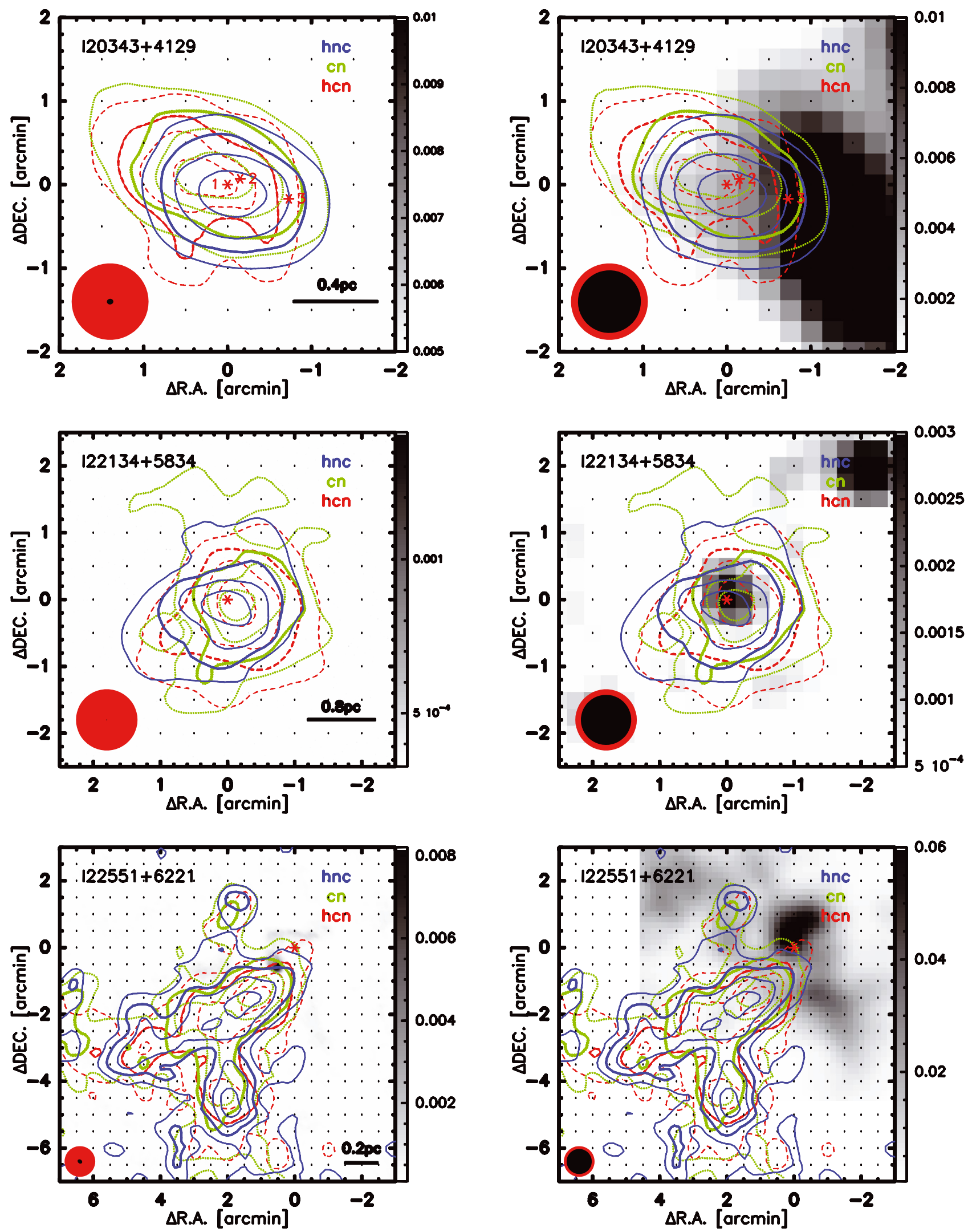

Fig. B.1. continued. 
A\&A 576, A131 (2015)
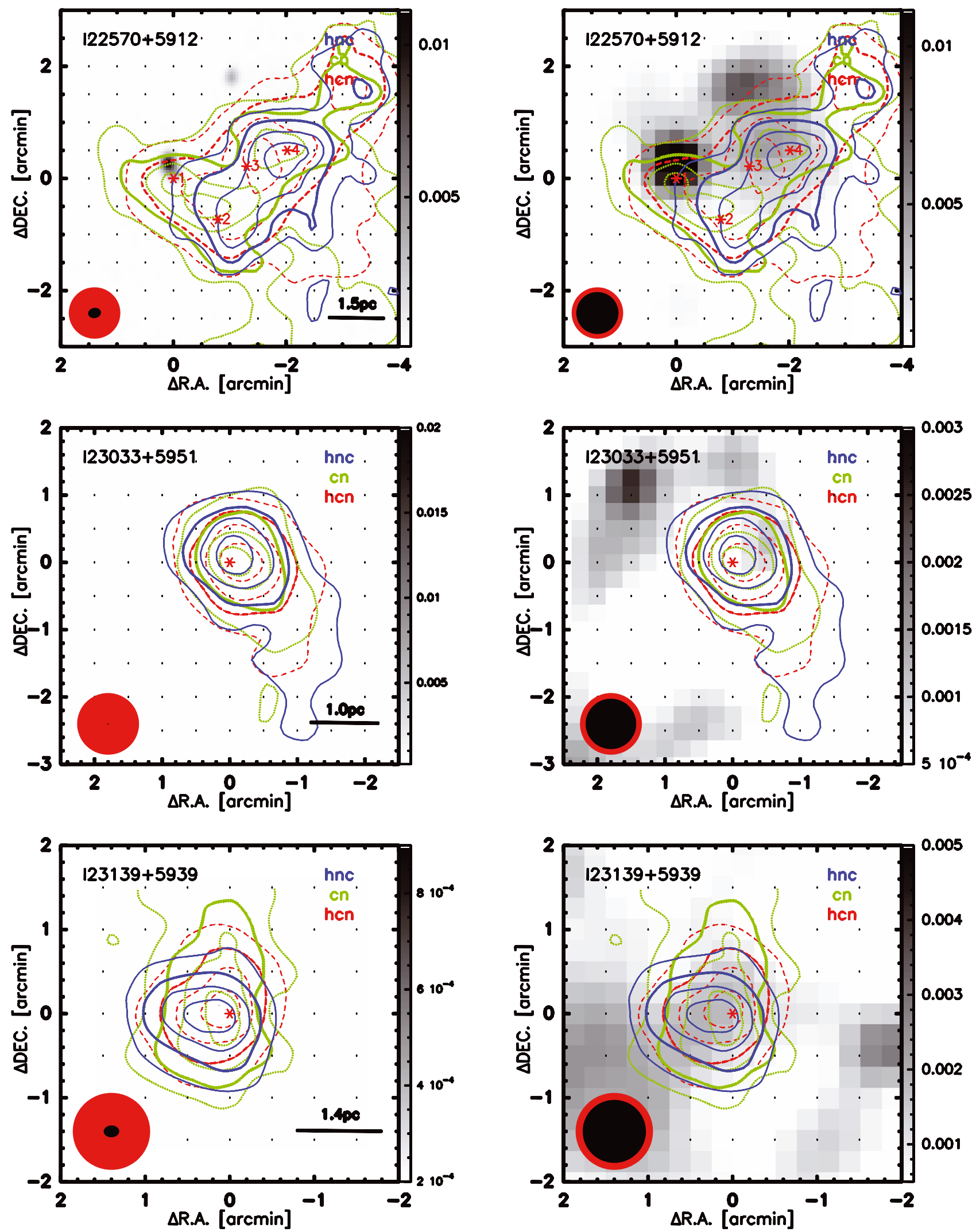

Fig. B.1. continued. 
X. H. Han et al.: A possible indicator for high-mass star formation sequence
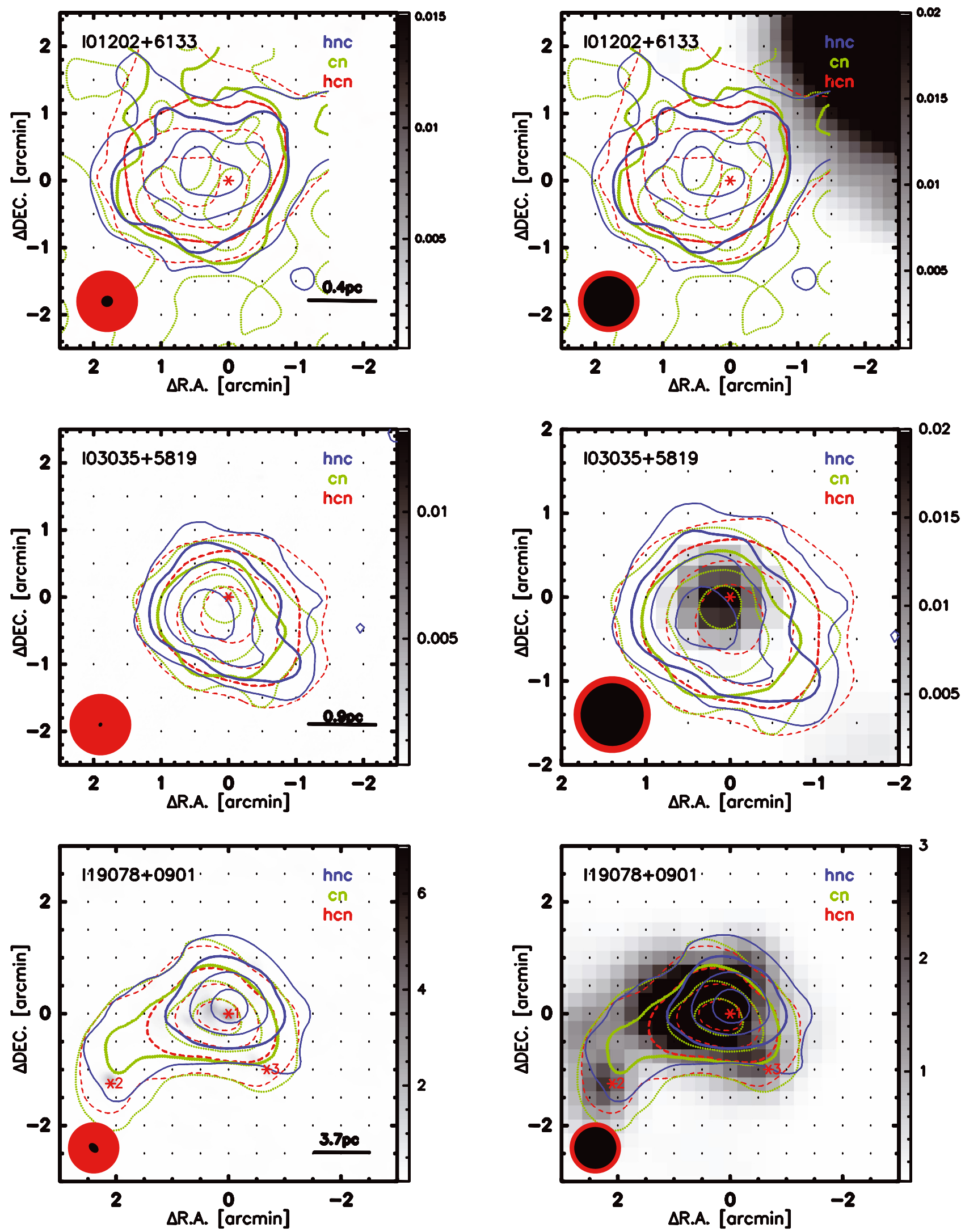

Fig. B.1. continued. 
A\&A 576, A131 (2015)
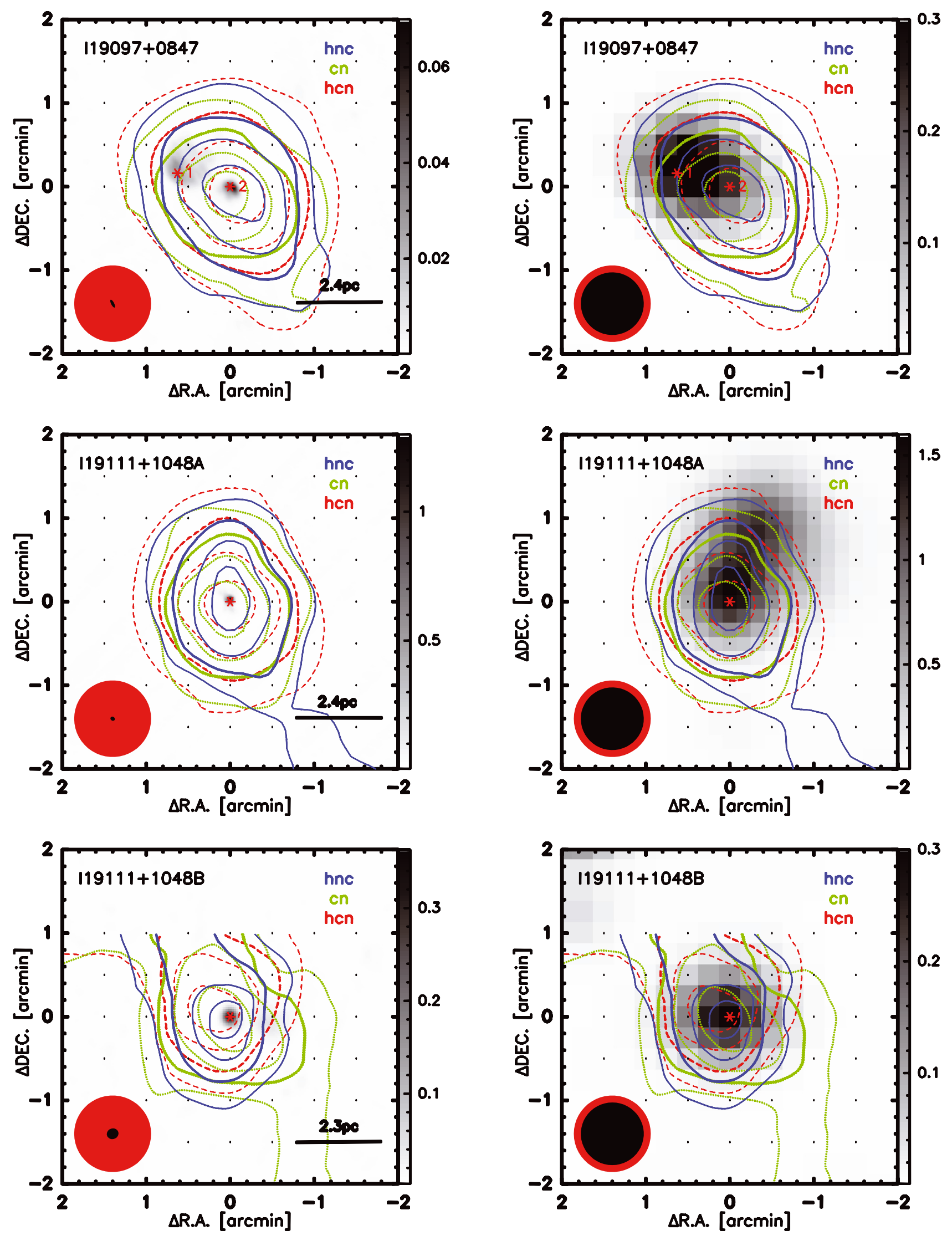

Fig. B.1. continued. 
X. H. Han et al.: A possible indicator for high-mass star formation sequence
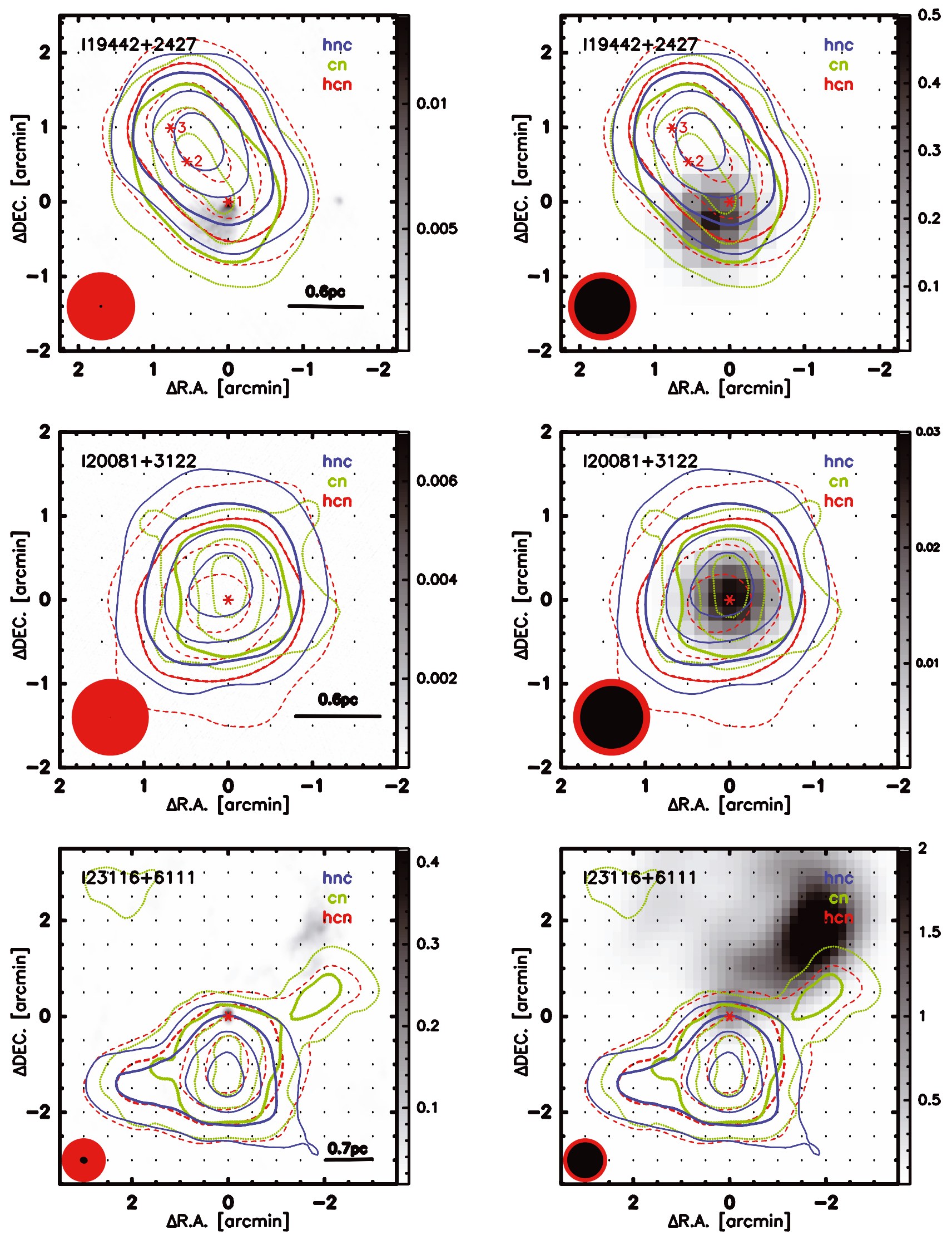

Fig. B.1. continued. 
A\&A 576, A131 (2015)
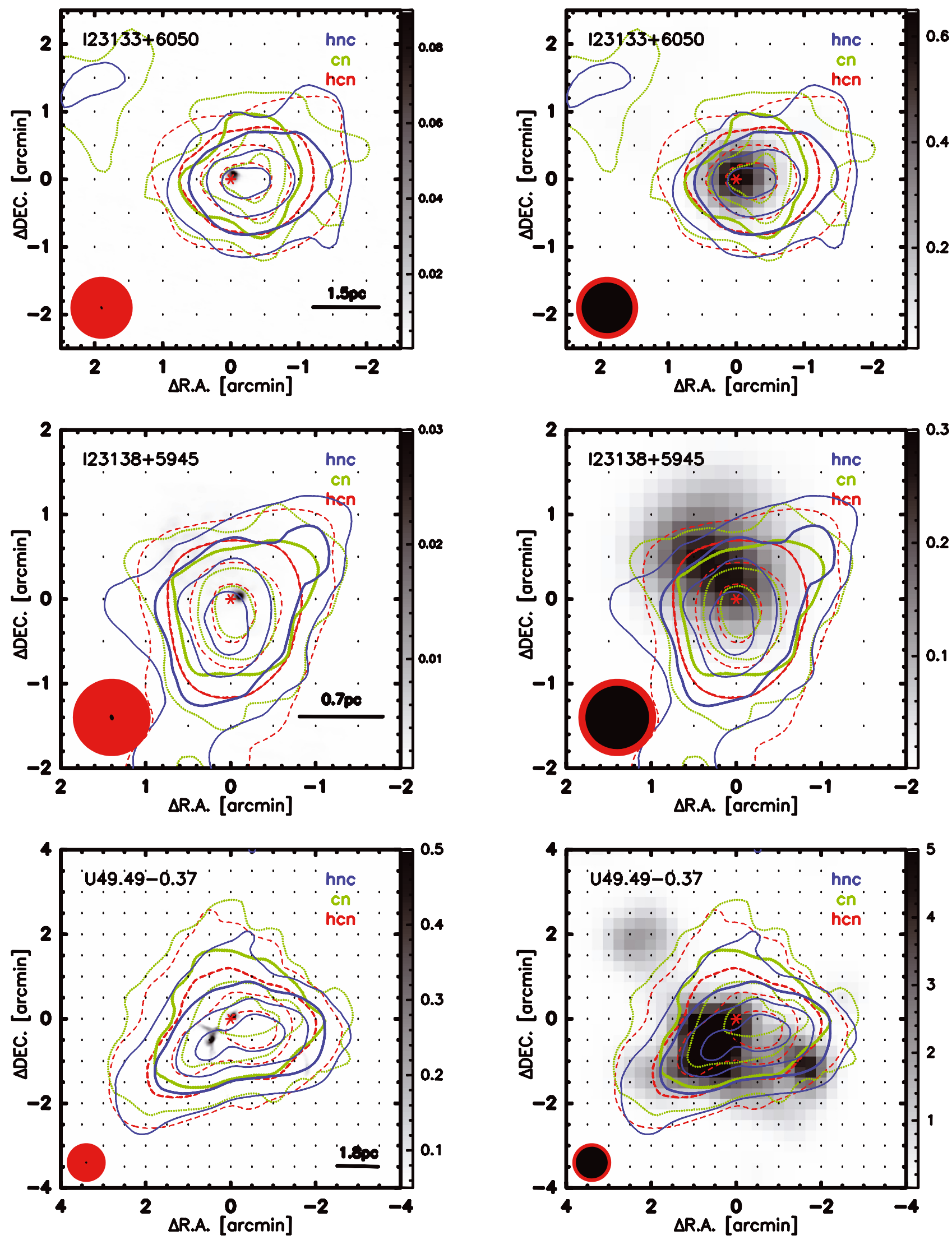

Fig. B.1. continued. 
X. H. Han et al.: A possible indicator for high-mass star formation sequence
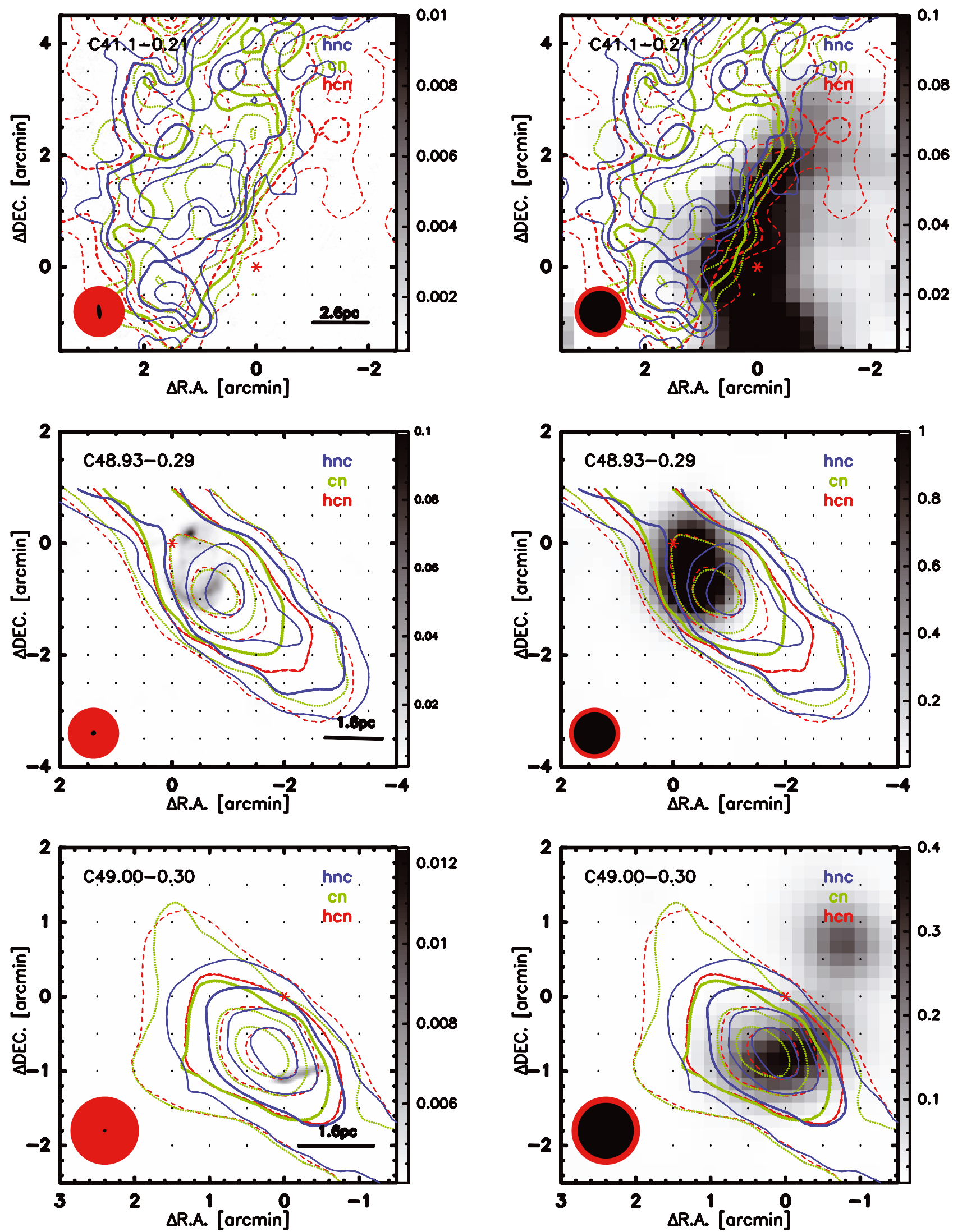

Fig. B.1. continued. 
A\&A 576, A131 (2015)
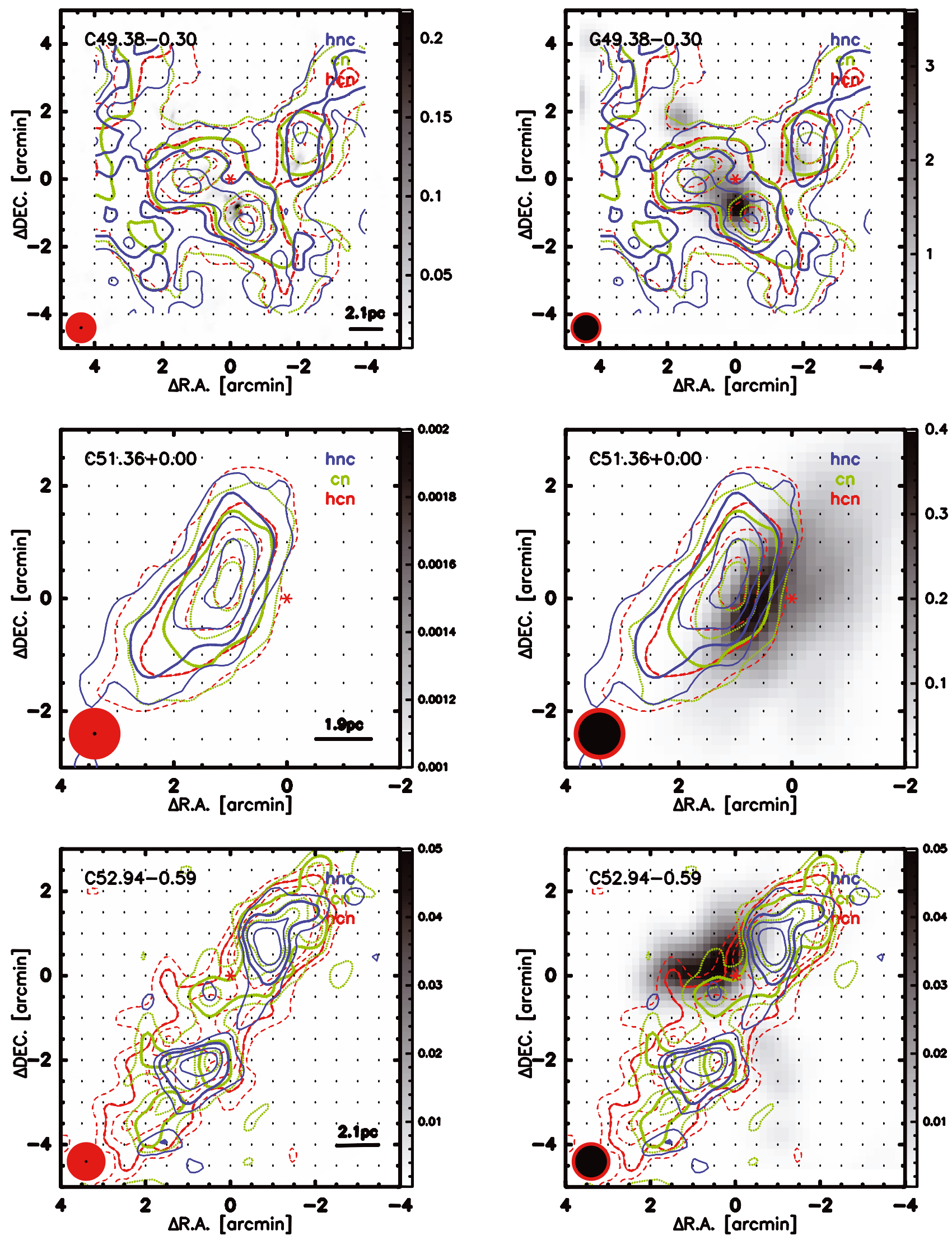

Fig. B.1. continued. 
X. H. Han et al.: A possible indicator for high-mass star formation sequence
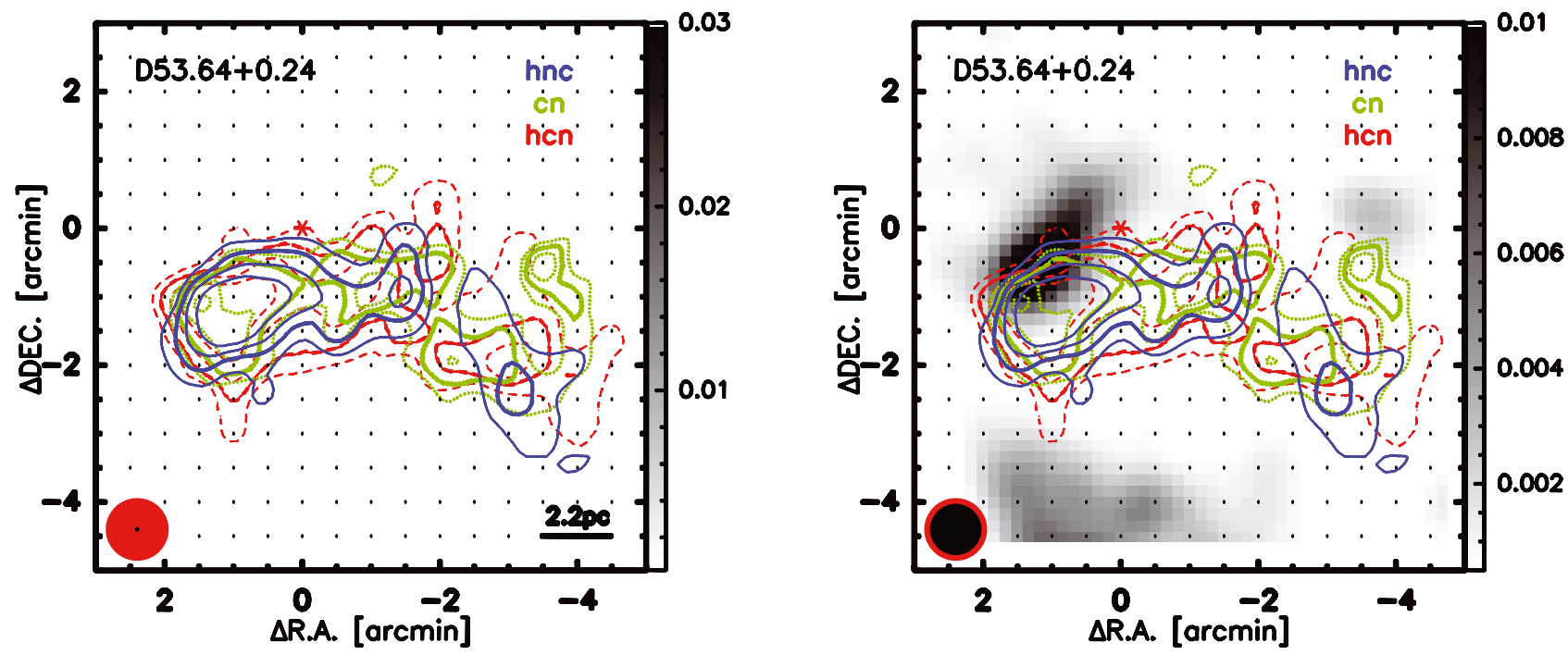

Fig. B.1. continued. 
A\&A 576, A131 (2015)

\section{Appendix C: $\mathrm{CN}(1-0), \mathrm{HCN}(1-0)$, and $\mathrm{HNC}(1-0)$ spectrum of all clumps}
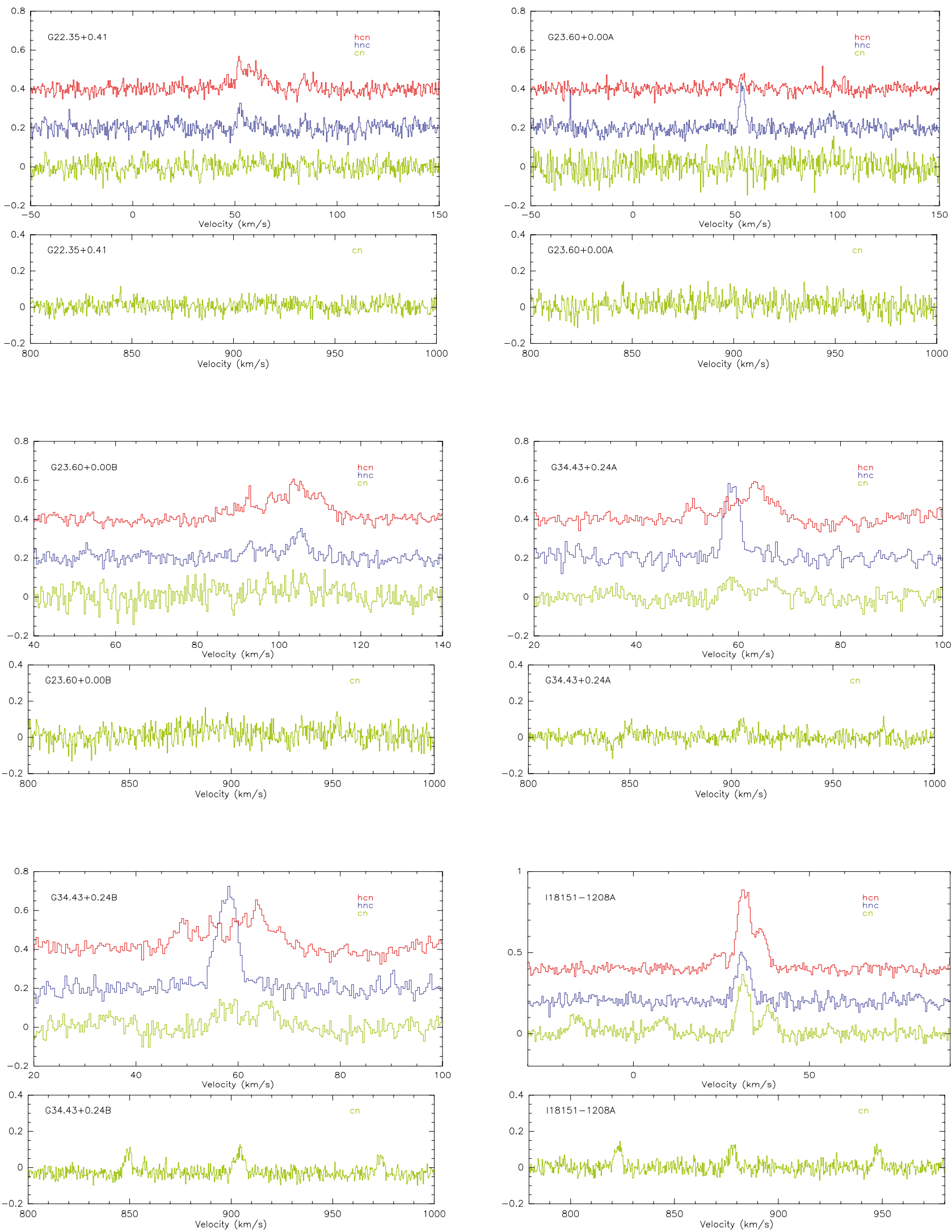

Fig. C.1. The $\mathrm{CN}(1-0), \mathrm{HCN}(1-0)$ and $\mathrm{HNC}(1-0)$ spectra averaged over each clump. 
X. H. Han et al.: A possible indicator for high-mass star formation sequence
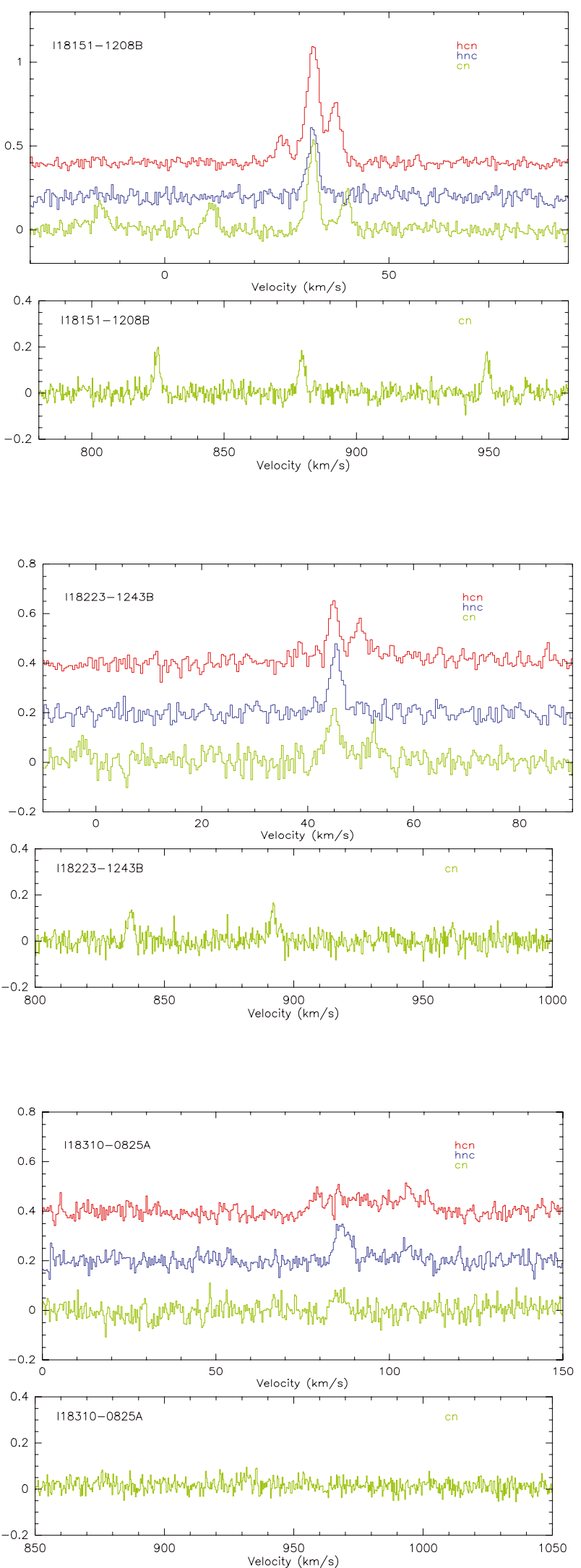

Fig. C.1. continued.
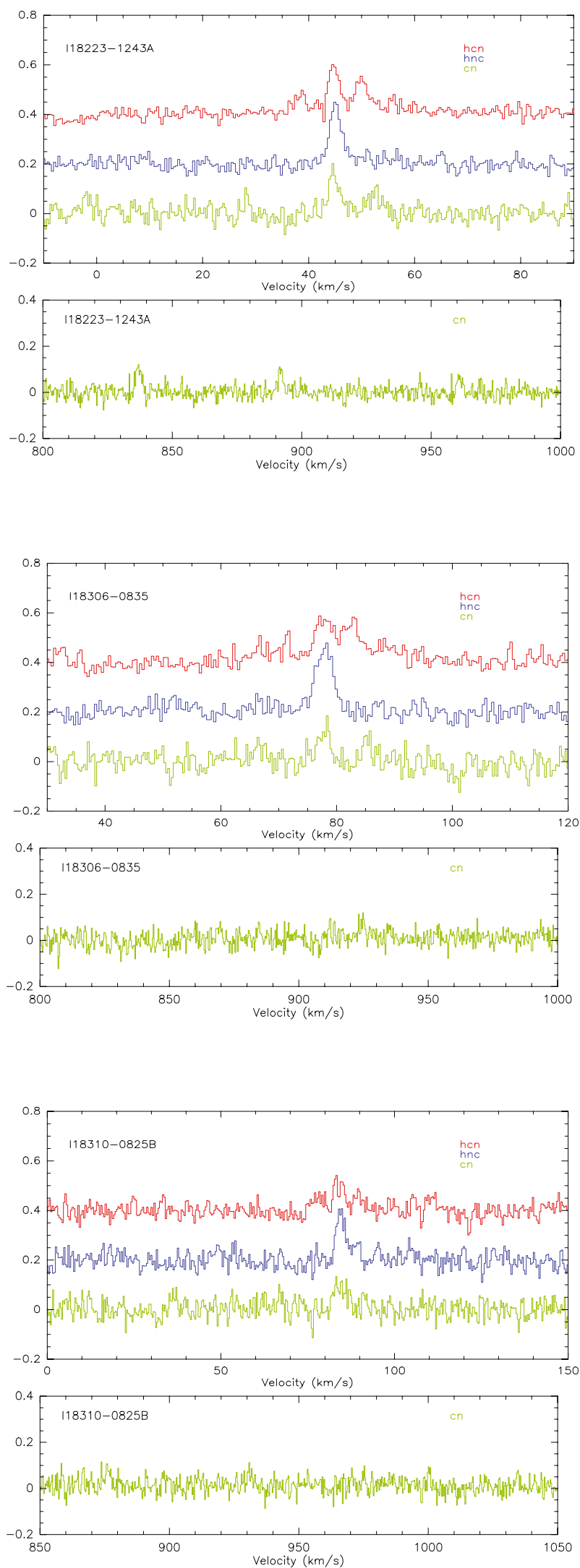
A\&A 576, A131 (2015)
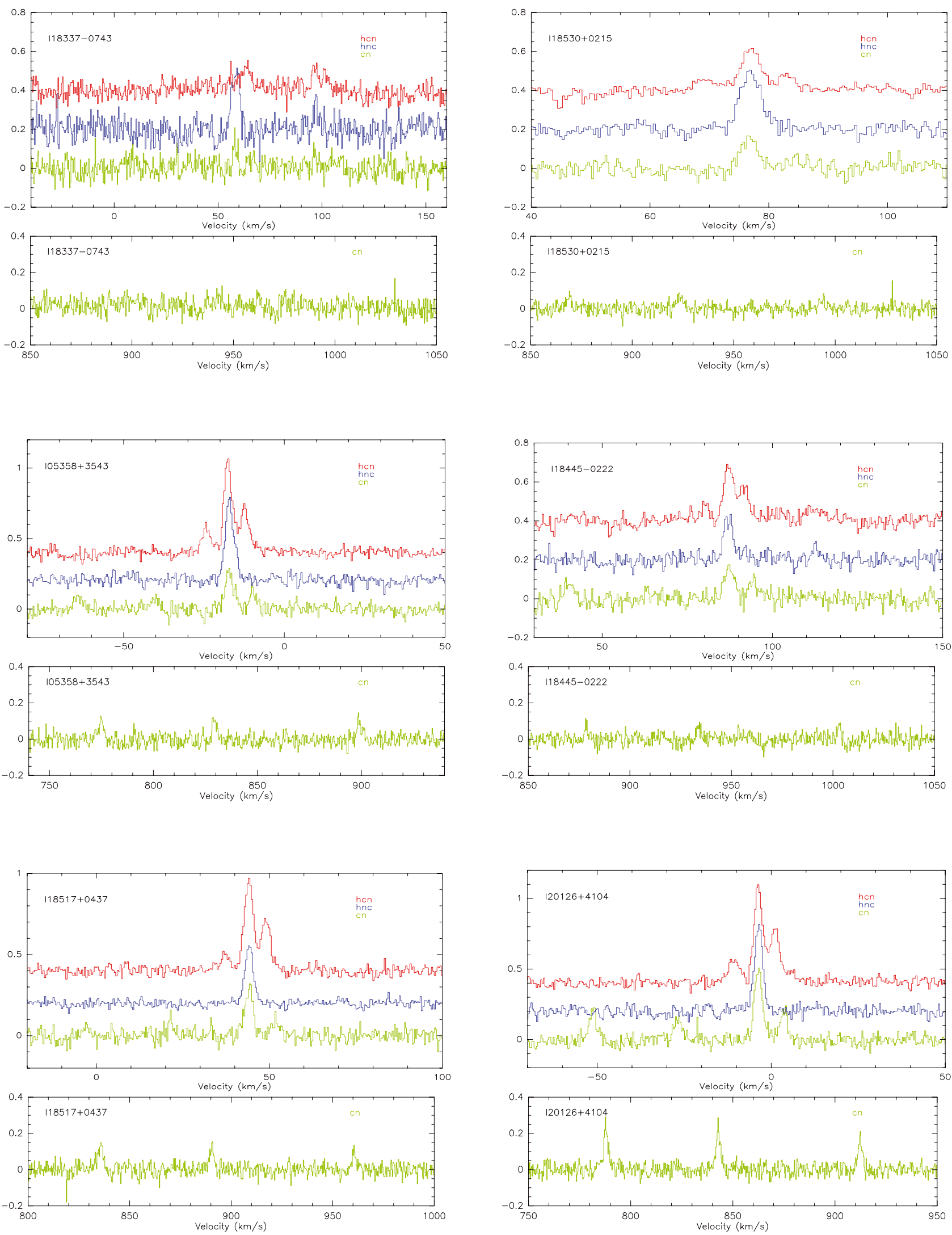

Fig. C.1. continued. 
X. H. Han et al.: A possible indicator for high-mass star formation sequence
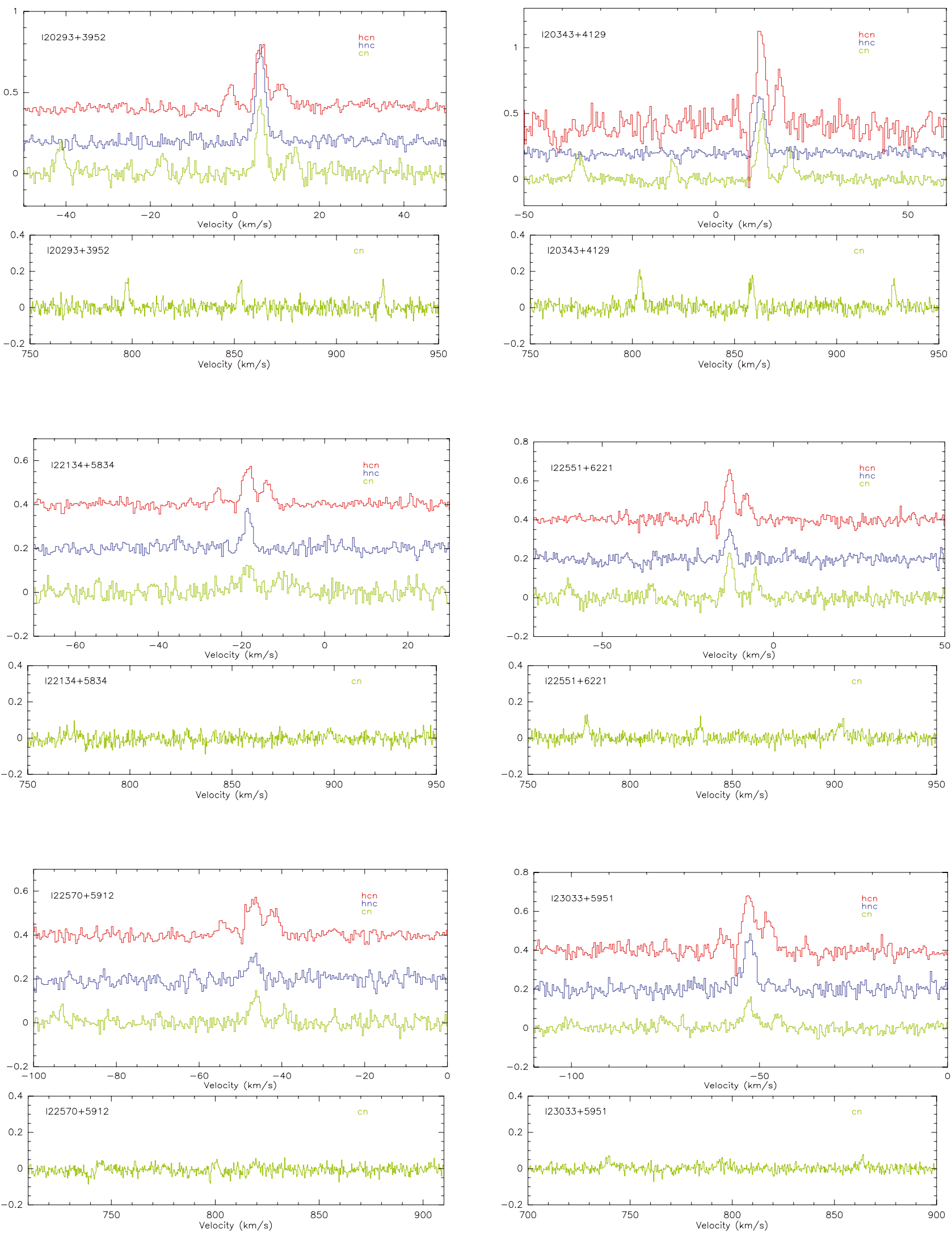

Fig. C.1. continued. 
A\&A 576, A131 (2015)
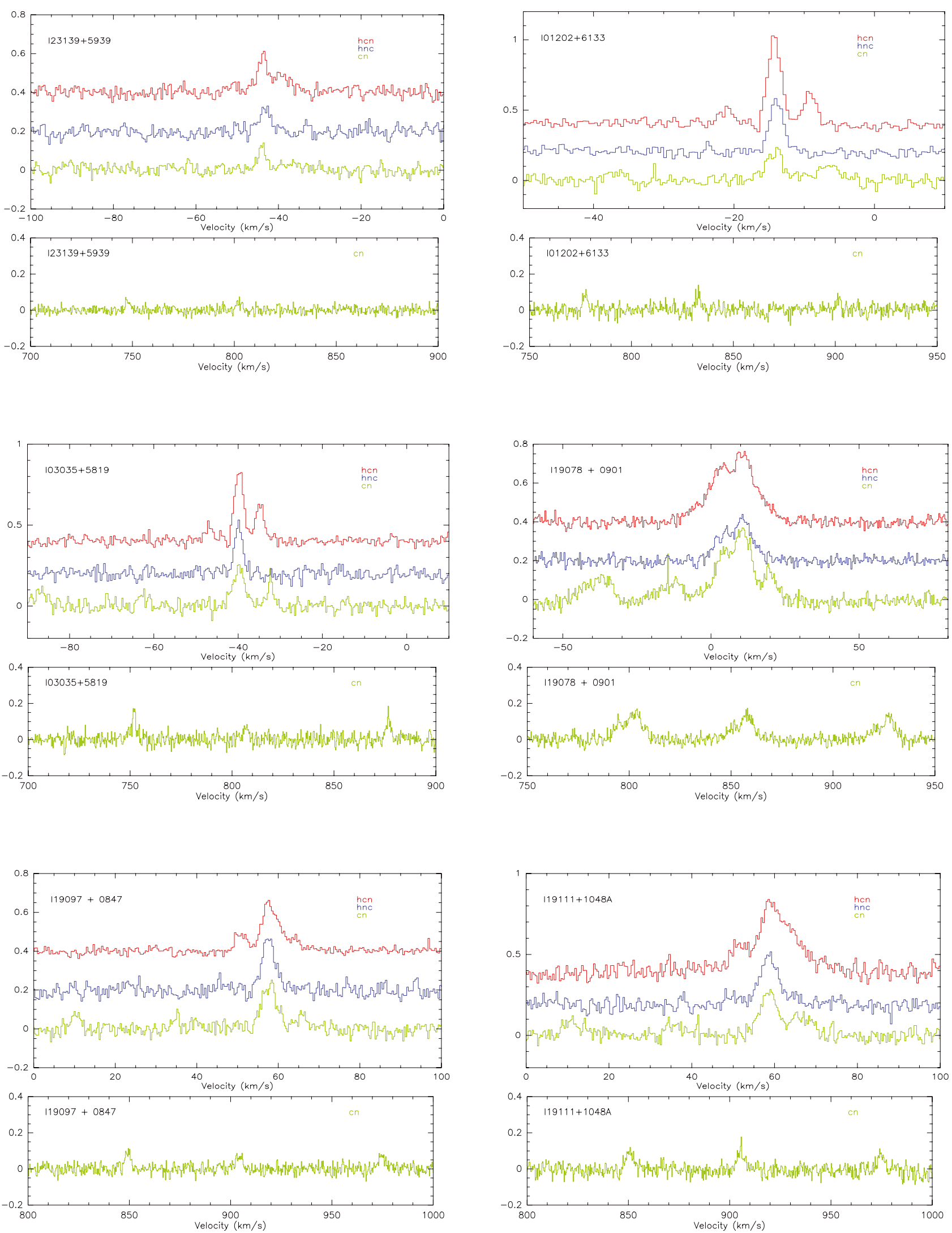

Fig. C.1. continued. 
X. H. Han et al.: A possible indicator for high-mass star formation sequence
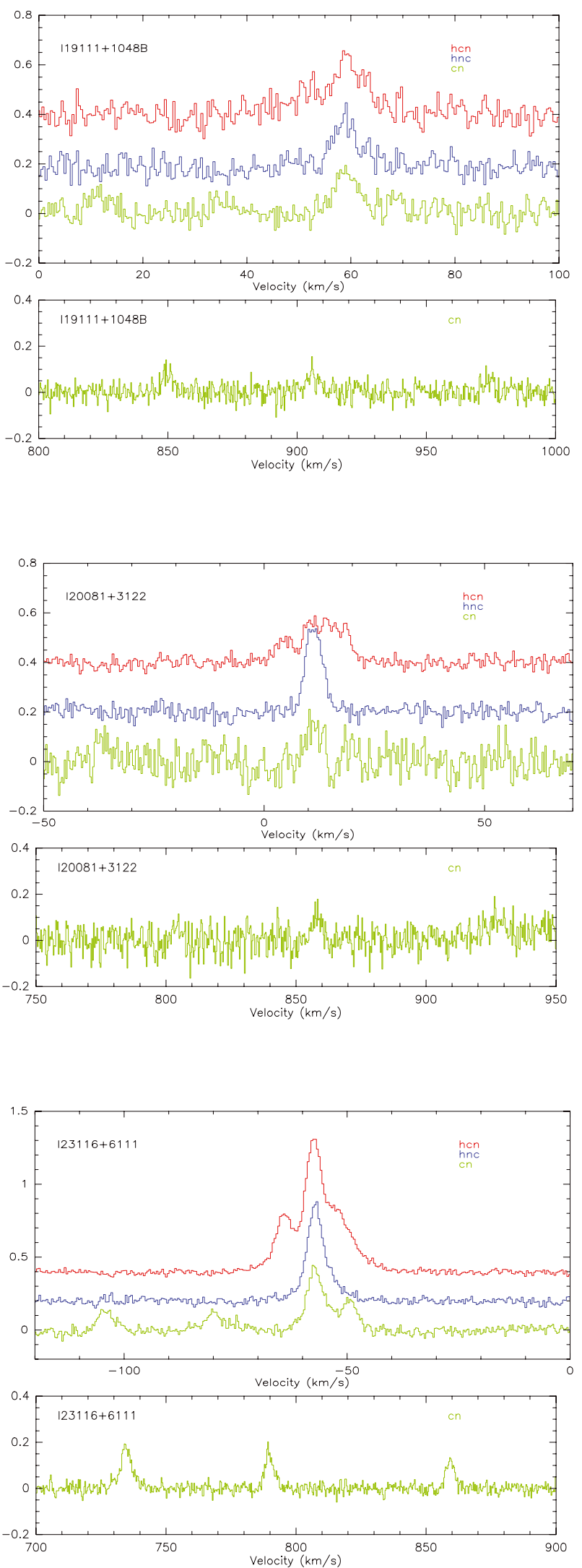

Fig. C.1. continued.
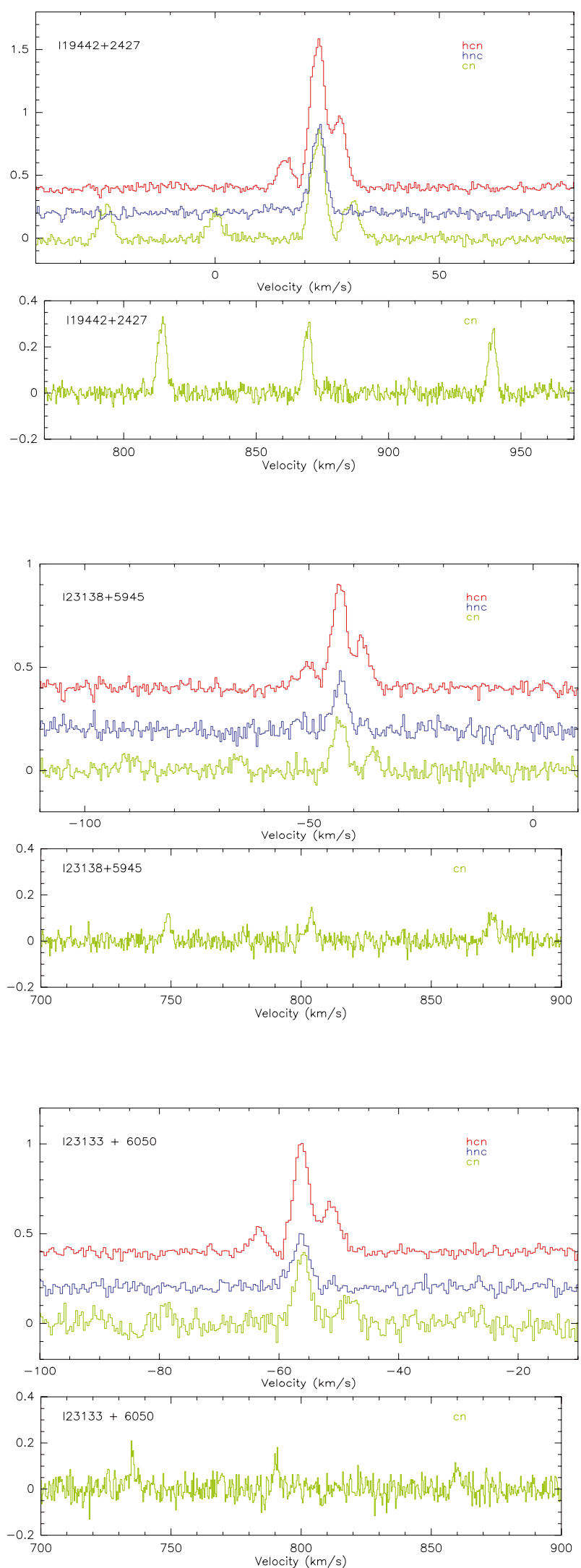
A\&A 576, A131 (2015)
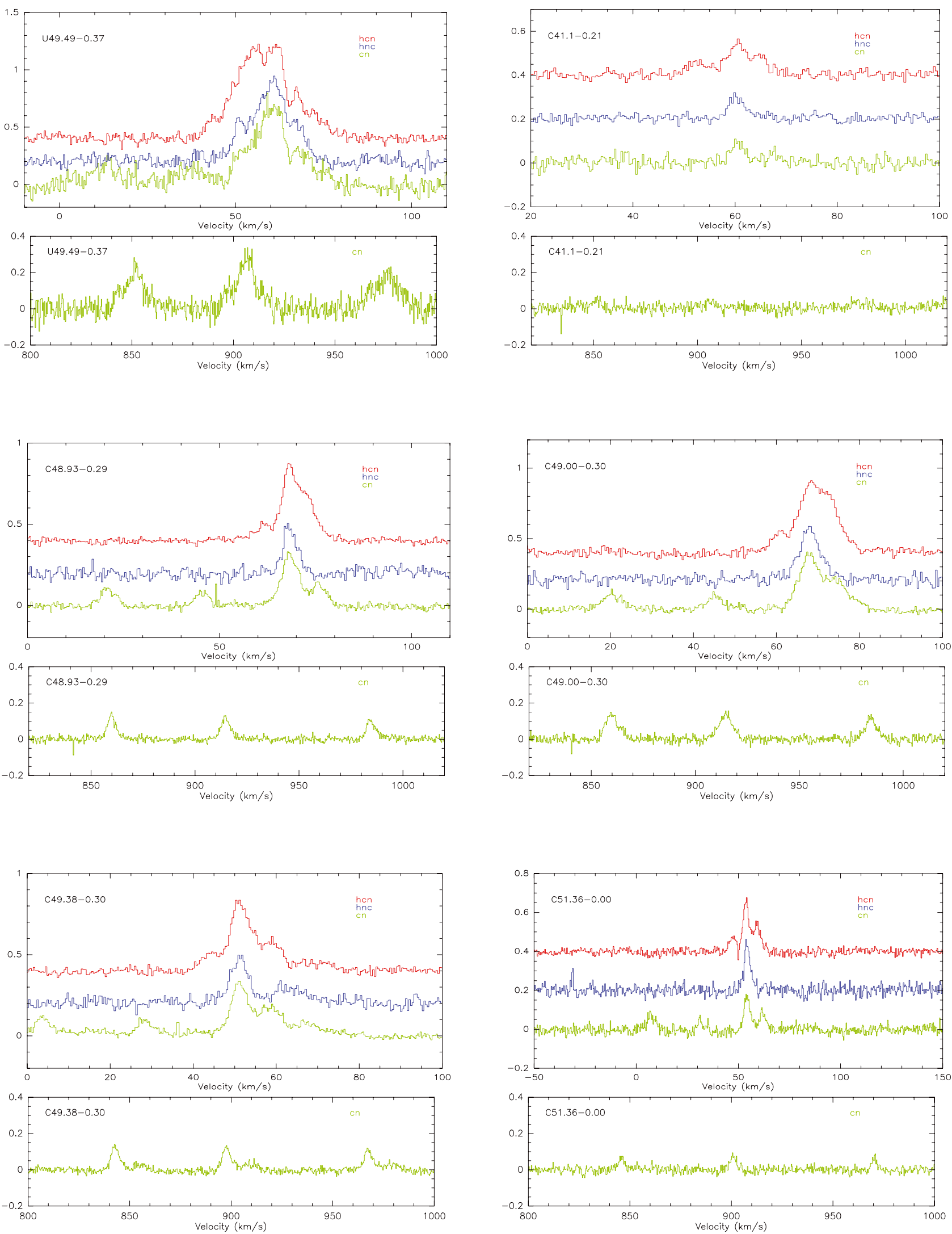

Fig. C.1. continued. 
X. H. Han et al.: A possible indicator for high-mass star formation sequence
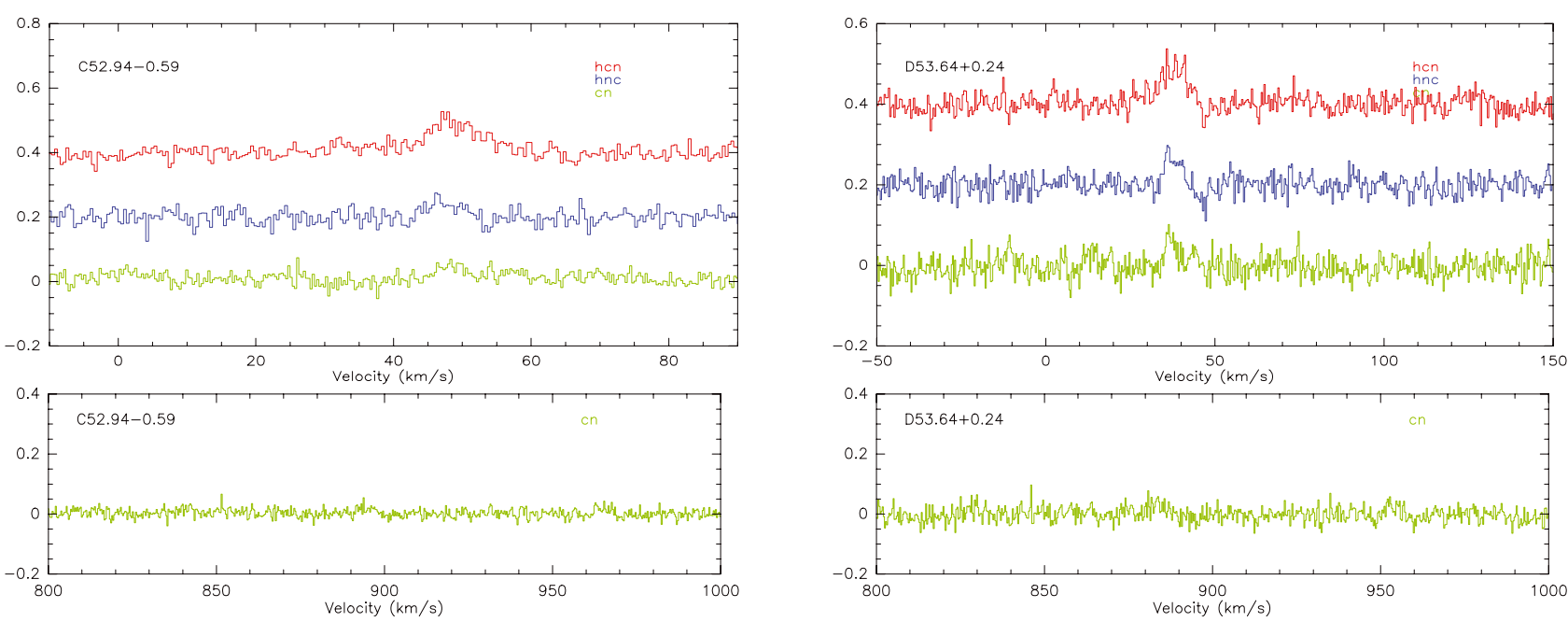

Fig. C.1. continued. 


\section{Appendix D: Detailed description of all clumps}

We performed CN(1-0), HCN(1-0) and HNC(1-0) map observations toward 38 high-mass star-forming regions with a size of $10^{\prime} \times 10^{\prime}$ and identified 44 high-mass clumps at different evolutionary stages. Below we briefly introduce all star-forming regions and molecular clumps.

G22.35+0.41-1: There is one small molecular clump in the mapped region, at the center of which the HMSC G22.35+0.41-1 is located. Hence the clump is classified as HMSCC (high-mass starless core clump). The western part of HMSCC G22.35+0.41 coincides with weak $20 \mathrm{~cm}$ continuum emission, which is an indicator for the presence of UV photons. This idea is supported by the detection of weak CN(1-0) emission that comes only from the western part of the clump.

G23.60+0.00-2: There are two molecular clumps in the mapped region. HMSCs G23.60+0.00-2, -4 and -7 lie in a small clump G23.60+0.00A, which is classified as HMSCC. HMSCC G23.60+0.00A coincides with the $20 \mathrm{~cm}$ continuum emission. HMSCs G23.60+0.00-1 and -3 and the UCHII region $\mathrm{G} 23.60+0.00-5$ lie in clump $\mathrm{G} 23.60+0.00 \mathrm{~B}$, and so the clump is classified as UCHIIC (ultra-compact HII region clump). G23.60+0.00B coincides with $20 \mathrm{~cm}$ continuum emission. $\mathrm{CN}(1-0)$ emission is strong around the UCHII region G23.60+0.00-5 and extends to the north. The line velocity of $\mathrm{G} 23.60+0.00 \mathrm{~A}$ is much lower than that of G23.60+0.00B (see Table 2), the two clumps are probably at different distances along the line of sight.

G34.43+0.24-3: Two molecular clumps are identified in the mapping region. HMSC G34.43+0.24-3, -6 , and -9 lie in clump $\mathrm{G} 34.43+0.24 \mathrm{~A}$, making G34.43+0.24A a HMSCC. There is no $20 \mathrm{~cm}$ continuum emission detected nearby the clump. HMPO G34.43+0.24-2 and HMSCs G34.43+0.24-1, -4, -5, and -8 also lie in clump G34.43+0.24B, the clump is therefore classified as HMPOC (high-mass protostar clump). There is weak $20 \mathrm{~cm}$ emission detected from the clump.

I18151-1208-2: There are two clumps in the mapped region, but they are not clearly separated. HMSC I18151-1208-2 is located at the center of the clump I18151-1208A, and so the clump is classified as HMSCC. HMPO I18151-1208-1 is located at the center of the clump I18151-1208B, classifying the clump as HMPOC. Both clumps coincide with $20 \mathrm{~cm}$ continuum emission.

I18223-1243-3: There are two clumps in the mapped region. HMSC I18223-1243-3 and HMSC I18223-1243-4 are located at the center and at the southern border of the clump I182231243A, respectively, making the clump an HMSCC. In addition, HMPO I18223-1243-1 is located at the center of the clump I18223-1243B. There is one relatively strong radio source at $3.6 \mathrm{~cm}$ continuum emission detected at the center of the clump, suggesting that there is one UCHII region, at I18223-1243B, classified as UCHIIC. The clump also partly coincides with strong $20 \mathrm{~cm}$ continuum emission that extends from the south to the north.

I18306-0835-3: One molecular clump was identified in the mapped region, I18306-0835. HMPO I18306-0835-1 is located at the center of the clump I18306-0835A. There is relatively strong continuum emission at $6 \mathrm{~cm}$ close to the HMPO, and together with the strong radio continuum emission source detected at $3.6 \mathrm{~cm}$ (Sridharan et al. 2002), this suggests that there is one UCHII region at the center of the clump. I18306-0835 is therefore classified as UCHIIC, and the clump coincides with the relatively weak and extended $20 \mathrm{~cm}$ continuum emission. The parental clump of HMSC I18306-0835-3 was not detected.
I18310-0825-4: There are two clumps in the mapped region. HMPO I18310-0825-2 and HMSCs I18310-0825-3 and -4 lie in the clump I18310-0825A, and HMPO I18310-0825-1 lies in the clump I18310-0825B classifying these two clumps as HMPOC. I18310-0825A partly coincides with $20 \mathrm{~cm}$ continuum emission, while I18310-0825B completely coincides with $20 \mathrm{~cm}$ continuum emission.

I18337-0743-3: There is only one dense molecular clump I18337-0743 identified in the mapped region, which contains HMPO I18337-0743-1 and HMSC I18337-0743-5. The clump is classified as HMPOC. Here the distribution of $\mathrm{CN}(1-0)$ is similar to that of $\mathrm{HCN}(1-0)$, but is different from that of $\mathrm{HNC}(1-0)$. I18337-0835-1 is located at the center of CN(1-0) and $\mathrm{HCN}(1-0)$ map. HMPOC I18337-0743 coincides with $20 \mathrm{~cm}$ continuum emission at its northeastern part. We did not detect the dense clump in which I18337-0743-3 has formed.

I18530+0215-2: One dense clump was identified in the mapped region. HMSC I18530+0215-2 lies on the southwestern border of the clump, while HMPO I18530+0215 is located at the center of it. There is strong continuum emission $(6$ and $20 \mathrm{~cm})$ close to the HMPO, and together with one detected radio source with strong continuum emission at $3.6 \mathrm{~cm}$ (Sridharan et al. 2002), this suggests that there is one UCHII region at the center of the clump, making the clump a UCHIIC.

I01202+6133: There is one molecular clump in the mapped region, with HMPO I01202+6133 located at the center. The clump is classified as HMPOC. HMPOC I01202+6133 partly coincides with $20 \mathrm{~cm}$ continuum emission.

I05358+3543: There is one dense molecular clump identified in the mapped region, in which HMPO I05358+3543-1 and -4, HMSCs I05358+3543-2 and -3 lie. HMPO I05358+3543-1 is located close to the center of the clump and shows very weak continuum emission at $6 \mathrm{~cm}$. We classify the clump $\mathrm{I} 05358+3543$ as HMPOC. There is no $20 \mathrm{~cm}$ continuum emission nearby it.

I18445-0222: There is one dense clump in the mapped region, HMPO I18445-0222-1 is located in the southern part of it. However, one radio source with relatively strong continuum emission at $3.6 \mathrm{~cm}$ was detected close to the HMPO (Sridharan et al. 2002), this suggests that there is one UCHII region at the center of the clump. Thus we classify the clump as UCHIIC. UCHIIC I18445-0222 partly coincides with very weak $20 \mathrm{~cm}$ continuum emission.

I18517+0437: There is one dense molecular clump in the mapped region, HMPO I18517+0437-1 is located at its center, and HMPO I18517+0437-2 lies to the northwest of the clump. We therefore classify the clump as HMPOC. HMPOC I18517+0437 partly coincides with very weak $20 \mathrm{~cm}$ continuum emission.

I20126+4104: There is one dense molecular clump in the mapped region, with HMPO I20126+4104 located at the center, showing very weak $3.6 \mathrm{~cm}$ continuum emission, which makes the clump a HMPOC. HMPOC I20216+4104 partly coincides with very weak $20 \mathrm{~cm}$ continuum emission.

I20293+3952: One dense molecular clump is present in the mapped region, at the center of which HMPO I20293+3952-1 is located. It shows very weak $3.6 \mathrm{~cm}$ continuum emission, and HMPO I20293+3952-2 lies to the north of it. So clump I $20293+3952$ is classified as HMPOC. There is no $20 \mathrm{~cm}$ continuum emission nearby it.

I20343+4129: The one dense molecular clump present in the mapped region contains HMPOs I20343+4129-1 and -2, which are located at the center of the clump and close to each other, whereas HMPO I20343+4129-3 lies to the west of the 
clump. Hence the clump is classified as HMPOC. HMPOC I20343+4129 partly coincides with $20 \mathrm{~cm}$ continuum emission. I22134+5834: The dense molecular clump in the mapped region, with HMPO I22134+5834 located at its center, can be classified as HMPOC. HMPOC I22134+5834 partly coincides with the weak $20 \mathrm{~cm}$ continuum emission. This indicates that some $\mathrm{O} / \mathrm{B}$ stars may exist close to the clump, and the idea is supported by $\mathrm{CN}(1-0)$ and $\mathrm{HCN}(1-0)$ emission that extends to the west and south.

I22551+6221: There is one molecular complex in the mapped region, HMPO I22551+6221 is located at the northwestern border of it. HMPO I22551+6221 seems to be accompanied by one UCHII reigon (Sridharan et al. 2002). The molecular complex coincides with strong and extended continuum emission at $20 \mathrm{~cm}$, which traces one large HII region. We thus classify the molecular complex I22551+6221 as HIIC (HII region clump).

I22570+5912: There is one molecular complex in the mapped region, within which HMPOs I22570+5912-1, -2, -4, and HMSC I22570+5912-3 are located. HMPO I22570+5912-1 appears to be accompanied by one UCHII region (Sridharan et al. 2002). The molecular complex coincides with strong and extended $20 \mathrm{~cm}$ continuum emission, which traces one large HII region. The distortion at the northern border of the complex indicates that it may be interacting with HII region. Hence we classify the complex as HIIC.

123033+5951: There is one dense molecular clump in the mapped region, with the HMPO I23033+5951 located at the center. The clump is classified as HMPOC. HMPOC I $23033+5951$ partly coincides with very weak $20 \mathrm{~cm}$ continuum emission.

I23139+5939: The dense molecular clump in the mapped region, with HMPO I23139+5939 located at the center, is classified as HMPOC. HMPOC I23139+5939 coincides es with very weak $20 \mathrm{~cm}$ continuum emission.

I03035+5819: There is one dense molecular clump identified in the mapped region, UCHII region I03035+5819 is located at its center and emits continuum emission at $3.6 \mathrm{~cm}$ and $20 \mathrm{~cm}$. The clump is classified as UCHIIC.

I19078+0901: There is one dense molecular clump in the mapped region, with UCHII regions I19078+0901-1, -2 and -3 in it, and they emit very strongly at $3.6 \mathrm{~cm}$ and $20 \mathrm{~cm}$. The clump is classified as UCHIIC.

I19097+0847: There is one dense molecular clump in the mapped region, in which UCHII regions I19097+0847-1 and -2 lie. They emit strongly at $3.6 \mathrm{~cm}$ and $20 \mathrm{~cm}$, classifying the clump as UCHIIC.

I19111+1048: There are two molecular clumps in the mapped region. UCHII region I19111+1048 is located at the center of clump I19111+1048A, and emits strongly at $3.6 \mathrm{~cm}$ and $20 \mathrm{~cm}$. The $20 \mathrm{~cm}$ continuum emission shows a cometary morphology. UCHII region I19110+1045 is located at the center of clump $\mathrm{I} 19111+1048 \mathrm{~B}$ and emits strongly at $3.6 \mathrm{~cm}$ and $20 \mathrm{~cm}$. These two clumps are both classified as UCHIIC.

I19442+2427: There is one dense molecular clump in the mapped region, in which the compact HII regions I19442+24271, HMSC I19442+2427-2, and HMPO I19442+2427-3 lie. UCHII region I19442+2427-1 emits strongly at $3.6 \mathrm{~cm}$ and $20 \mathrm{~cm}$. We classify the clump as UCHIIC.

I20081+3122: The dense molecular clump in the mapped region contains UCHII region $\mathrm{I} 20081+3122$, which is located at the center of the clump and emits at $6 \mathrm{~cm}$ and $20 \mathrm{~cm}$. We classify the clump as UCHIIC.

I23116+6111: There is one dense molecular clump in the mapped region, at whose northern border lies UCHII region I23116+6111. It emits strongly at $3.6 \mathrm{~cm}$ and $20 \mathrm{~cm}$. However, strong and extended $3.6 \mathrm{~cm}$ and $20 \mathrm{~cm}$ continuum emission suggests that one large HII region lies to the north of the clump and appears to be interacting with the molecular clump. Hence we classify the clump as HIIC.

I23138+5945: There is one dense molecular clump in the mapped region, UCHII region $\mathrm{I} 23138+5945$ is located at its center and emits strongly at $3.6 \mathrm{~cm}$ and $20 \mathrm{~cm}$, with the latter emission shows a cometary morphology. We classify the clump as UCHIIC.

I23133+6050: One dense molecular clump was identified in the mapped region, UCHII region $\mathrm{I} 23133+6050$ is located at its center and emits strongly at $3.6 \mathrm{~cm}$ and $20 \mathrm{~cm}$. We classify the clump as UCHIIC.

U49.49-0.37: There is one very dense molecular clump in the mapped region, UCHII region U49.49-0.37 lies in it and emits strongly at $6 \mathrm{~cm}$ and $20 \mathrm{~cm}$. The $\mathrm{CN}(1-0)$ and $\mathrm{HCN}(1-0)$ emission extends to the northeast. We classify the clump as UCHIIC.

C41.1-0.21: There is one diffuse molecular complex in the mapped region. The HII region traced by $20 \mathrm{~cm}$ continuum emission shows a shell morphology and seems to be interacting with the molecular complex. The molecular complex is classified as HIIC.

C48.93-0.29: There is one dense molecular clump in the mapped region, where the HII region traced by $6 \mathrm{~cm}$ and $20 \mathrm{~cm}$ continuum emission coincides with the northeastern part of the clump. This HII region appears to be still surrounded by the clump. We classify the molecular clump as HIIC.

C49.00-0.30: The HII region traced by $20 \mathrm{~cm}$ continuum emission shows a shell morphology. The molecular clump partly coincides with the HII region and seems to be intersecting with it at a nearly right angle. The $\mathrm{CN}(1-0)$ and $\mathrm{HCN}(1-0)$ emission extends to the northeast. We classify the molecular clump as HIIC.

C49.38-0.30: There is one dense molecular complex in the mapped region, which is composed of several dense clumps. The HII region traced by $6 \mathrm{~cm}$ and $20 \mathrm{~cm}$ continuum emission shows a V-like morphology, which is consistent with the characteristics of the distribution of dense molecular clumps. The complex is classified as HIIC.

C51.36-0.00: There is one dense molecular clump in the mapped region. It partly coincides with the HII region traced by $20 \mathrm{~cm}$ continuum emission. The clump is classified as HIIC.

C52.94-0.59: There is one diffuse molecular complex in the mapped region with very weak $\mathrm{CN}(1-0), \operatorname{HCN}(1-0)$, and HNC(1-0) emission. The HII region traced by $20 \mathrm{~cm}$ continuum emission shows an arc morphology and seems to be interacting with the molecular complex. The complex is therefore classified as HIIC.

D53.64+0.24: There is one diffuse molecular complex in the mapped region with very weak $\mathrm{CN}(1-0), \operatorname{HCN}(1-0)$, and HNC(1-0) emission. The HII region, as shown by tracing the $20 \mathrm{~cm}$ continuum emission, seems to surround the complex. The complex is classified as HIIC. 